\title{
MATROIDS OF GAIN GRAPHS IN APPLIED DISCRETE GEOMETRY
}

\begin{abstract}
SHIN-ICHI TANIGAWA
ABSTRACT. A $\Gamma$-gain graph is a graph whose oriented edges are labeled invertibly from a group $\Gamma$. Zaslavsky proposed two matroids associated with $\Gamma$-gain graphs, called frame matroids and lift matroids, and investigated linear representations of them. Each matroid has a canonical representation over a field $\mathbb{F}$ if $\Gamma$ is isomorphic to a subgroup of $\mathbb{F}^{\times}$in the case of frame matroids or $\Gamma$ is isomorphic to an additive subgroup of $\mathbb{F}$ in the case of lift matroids. The canonical representation of the frame matroid of a complete graph is also known as a Dowling geometry, as it was first introduced by Dowling for finite groups $\Gamma$.

In this paper, we extend these matroids in two ways. The first one is extending the rank function of each matroid, based on submodular functions over $\Gamma$. The resulting rank function generalizes that of the union of frame matroids or lift matroids. Another one is extending the canonical linear representation of the union of $d$ copies of a frame matroid or a lift matroid, based on linear representations of $\Gamma$ on a $d$-dimensional vector space. We show that linear matroids of the latter extension are indeed special cases of the first extension, as in the relation between Dowling geometries and frame matroids. We also discuss an attempt to unify the extension of frame matroids and that of lift matroids.

This work is motivated by recent results in the combinatorial rigidity of symmetric graphs. As special cases, we give several new results on this topic, including combinatorial characterizations of the symmetry-forced rigidity of generic body-bar frameworks with point group symmetries or crystallographic symmetries and the symmetric parallel redrawability of generic bar-joint frameworks with point group symmetries or crystallographic symmetries.
\end{abstract}

\section{INTRODUCTION}

A $\Gamma$-gain graph $(G, \psi)$ is a pair of a graph $G=(V, E)$ and an assignment $\psi$ of an element of a group $\Gamma$ with each oriented edge such that reversing the direction inverts the assigned element. Gain graphs are also known as group-labeled graphs. Zaslavsky [44 46] studied a class of matroids of graphs, called frame matroids (formerly known as bias matroids), and as a principal subcase he considered a matroid associated with a $\Gamma$-gain graph $(G, \psi)$, called the frame matroid $\mathbf{F}(G, \psi)$. Frame matroids include several known matroids, such as graphic matroids, bicircular matroids, Dowling geometries, and matroids on signed graphs. Zaslavsky [45] also proposed another matroid associated with a $\Gamma$-gain graph $(G, \psi)$, called the lift matroid $\mathbf{L}(G, \psi)$, which can be constructed from the graphic matroid of $G$ by an elementary lift.

Received by the editors November 9, 2012 and, in revised form, October 18, 2013.

2010 Mathematics Subject Classification. Primary 52C25, 05B35; Secondary 05C75, 05C10, $68 \mathrm{R} 10$.

This work was supported by JSPS Grant-in-Aid for Young Scientist (B), 24740058. 
$\mathbf{F}(G, \psi)$ and $\mathbf{L}(G, \psi)$ both have a canonical representation over a field $\mathbb{F}$ if $\Gamma$ is isomorphic to a subgroup of the multiplicative group $\mathbb{F}^{\times}$of $\mathbb{F}$ in the case of $\mathbf{F}(G, \psi)$ or $\Gamma$ is isomorphic to an additive subgroup of $\mathbb{F}$ in the case of $\mathbf{L}(G, \psi)$. The canonical representation of the frame matroid of a dense graph is also known as a Dowling geometry, as it was first introduced by Dowling [7].

As a further extension, Whittle 43 discussed a counterpart of frame matroids in general matroids by extending the construction of frame matroids from graphic matroids.

In this paper, we shall consider extensions, sticking to gain graphs. We propose matroids of gain graphs, extending the constructions of frame matroids or lift matroids in the following two ways. The first one is extending the rank function of each matroid, based on submodular functions over $\Gamma$. The resulting rank function generalizes that of the union of frame matroids or lift matroids. Another one is extending the canonical linear representation of the union of $d$ copies of a frame matroid or a lift matroid, based on linear representations of $\Gamma$ on a $d$-dimensional vector space. We show that linear matroids of the latter extension are indeed special cases of the first extension, as in the relation between Dowling geometries and frame matroids.

1.1. Applications to rigidity theory. This work is motivated by recent results in the combinatorial rigidity of symmetric graphs, and most parts of this paper are devoted to this application.

Characterizing generic rigidity of graphs is one of the central problems in rigidity theory, where a graph is identified with a bar-joint framework by regarding each vertex as a joint and each edge as a bar in the Euclidean space (see, e.g., 42]). In this context, a bar-joint framework is denoted by a pair $(G, p)$ of a graph $G=(V, E)$ and $p: V \rightarrow \mathbb{R}^{d}$. For 2-dimensional rigidity, Laman's theorem [18 (along with a result by Asimov and Roth [1] or Gluck [13]) asserts that $(G, p)$ is minimally rigid on any generic $p: V \rightarrow \mathbb{R}^{2}$ if and only if $|E|=2|V|-3$ and $|F| \leq 2|V(F)|-3$ for any nonempty $F \subseteq E$, where $V(F)$ denotes the set of vertices incident to edges in $F$. However, despite exhausting efforts so far, the 3-dimensional counterpart has not been obtained yet.

Although characterizing generic 3-dimensional rigidity of graphs is recognized as one of the most difficult open problems in this field, there are solvable structural models even in higher dimension. The most important case is a body-bar framework introduced by Tay [39]. A body-bar framework is a structural model consisting of disjoint rigid bodies articulated by bars, and the underlying graph is extracted by associating each body with a vertex and each bar with an edge. Tay [39] proved that a generic body-bar framework (i.e., relative positions of bars are generic) is rigid if only if the underlying graph has rank $\left(\begin{array}{c}d+1 \\ 2\end{array}\right)(|V|-1)$ in the union of $\left(\begin{array}{c}d+1 \\ 2\end{array}\right)$ copies of the graphic matroid.

Building up mathematical models of oscillations of chemical compounds or phase transitions of crystal materials is one of the main issues in theoretical physics, and toward understanding topological impacts in such phenomena there are attempts to extend those theorems for generic rigidity to symmetric frameworks in the past few years. Here, symmetric frameworks are those which are invariant under the action of a point group in the finite case or of a space group in the infinite case. The papers by Borcea and Streinu [3, Power [28, or Schulze et al. 35] demonstrate 
applications of the theory to specific ideal crystals or proteins and discuss possible extensions.

For a finite case, initiated by a combinatorial necessary condition [6, 10, Schulze [32,33. showed an extension of Laman's theorem of minimal 2-dimensional rigidity subject to certain point group symmetries.

Characterizing symmetry-forced rigidity, proposed for finite frameworks in 36 and for infinite periodic frameworks in [3, 4, is now recognized as an important initial step to understand the rigidity of symmetric frameworks, where in this model each motion is also subject to the underlying symmetry. (For other attempts to capture the flexibility of periodic frameworks, see, e.g., [25, 26].) It was proved that the symmetry-forced generic rigidity (i.e., symmetry-forced rigidity on generic configurations subject to the symmetry) can be checked by computing the rank of linear matroids defined on the edge sets of the underlying quotient gain graphs and thus can be analyzed in a conventional manner. Symmetry-forced rigidity was characterized in terms of the underlying quotient gain graphs by Ross [29, 30. for periodic 2-dimensional bar-joint frameworks and periodic 3-dimensional bodybar frameworks with fixed lattice metric and by Malestein and Theran 21,22 for crystallographic 2-dimensional bar-joint frameworks with flexible lattice metric.

The result of this paper is indeed inspired by these previous results. As shown by Lovász and Yemini [20, Tay [39] and Whiteley [40, 42, the union of copies of graphic matroids plays a central role in combinatorial rigidity theory; that is, most combinatorial characterizations are written in terms of the union of copies of graphic matroids or its variants, called count matroids (see, e.g., [1] for count matroids). It is thus natural to investigate the union of copies of frame matroids or lift matroids to derive the symmetric analogues on gain graphs. However, when compared with the canonical linear representation of the union of frame matroids (cf. 93 ), linear matroids of gain graphs proposed in the context of rigidity [4, 5, 21, 30, 36, greatly rely on algebraic structures of the underlying groups. The primary motivation of this paper is to propose a new class of matroids of gain graphs, which forms the foundation in the study of symmetry-forced rigidity, as does the union of graphic matroids in the classical rigidity problem.

As another application, we shall also consider the symmetric version of the parallel redrawing problem of graphs. In the parallel redrawing problem, we are asked whether a given straight-line drawing of a graph admits a parallel redrawing, that is, another straight-line drawing such that each edge is parallel to the corresponding one in the original drawing. Since any drawing admits a parallel redrawing by a translation or a dilation, we are asked whether all possible parallel redrawings are obtained in these trivial ways. In 42 Whiteley proved a combinatorial characterization for parallel redrawability of generic drawings. Here, we shall discuss the symmetric counterpart, called the symmetric parallel redrawing problem, where both drawing and its redrawing are subject to symmetry.

Similar matroids arose in scene analysis; see Whiteley [42. Replacing the union of graphic matroids with our new matroids, it is possible to extend the characterizations to the symmetric version.

We list applications addressed in this paper: the $d$-dimensional symmetric parallel redrawing problem with point group symmetry ( $(6.3)$; the 2-dimensional symmetry-forced rigidity of bar-joint frameworks with rotational symmetry ( $\$ 6.4)$; the $d$-dimensional symmetry-forced rigidity of body-bar frameworks with point 
group symmetry or crystallographic symmetry with fixed lattice metric (\$7.2); the $d$-dimensional symmetric parallel redrawing problem with crystallographic symmetry with flexible lattice metric (\$10.2); the 2-dimensional symmetry-forced rigidity of bar-joints frameworks with crystallographic symmetry whose linear part is a group of rotations ( $₫ 10.2$ ). The results provide alternative proofs of existing works as well as new statements, which solve questions (explicitly/implicitly) posed in $[21,29,30,36]$.

1.2. Organization. The paper is organized as follows. In $₫ 2$ and $₫ 3$, we briefly review fundamental facts on gain graphs and (poly)matroids, respectively. In particular, we shall explain details of matroids induced by monotone submodular functions in $\$ 3$, as our extensions belong to this class.

Extensions of frame matroids and lift matroids are described in 9 , \$5 , 89 and 99, and the remaining sections are devoted to applications. In \$4, we give an extension of rank functions of frame matroids via submodular functions over groups, while in 55 we give an extension of Dowling geometries via group representations. We give a combinatorial characterization (Theorem 5.4) of the proposed linear matroids, which implies that these linear matroids are special cases of matroids combinatorially defined in $\$$. Proving such a characterization does not look an easy task at a glance, but it turns out, by using the polymatroid theory discussed in 93 , that the problem is as easy as the case of frame matroids.

As applications, we will discuss the parallel redrawing problem and the symmetryforced rigidity of bar-joint frameworks with point group symmetry in $\$ 6$. In \$7 we also discuss an application to the symmetry-forced rigidity of body-bar frameworks with crystallographic symmetry.

In $₫ 8$, we give counterparts of those results for lift matroids. In 99 , we attempt to unify the extension of frame matroids and that of lift matroids, based on the representation theory obtained so far. In \$10, we give further applications to the parallel redrawing problem or the rigidity problem of bar-joint frameworks with crystallographic symmetry.

1.3. Basic definitions and notation. A partition $\mathcal{P}$ of a finite set $E$ is a set of nonempty subsets of $E$ such that each element of $E$ belongs to exactly one subset of $\mathcal{P}$. If $E=\emptyset$, the partition of $E$ is defined as the empty set. A subpartition of $E$ is a partition of a subset of $E$.

For an undirected graph $G, V(G)$ and $E(G)$ denote the vertex set and the edge set of $G$, respectively. For $F \subseteq E(G), V(F)$ denotes the set of endvertices of edges in $F$, and $G[F]=(V(F), F)$, that is, the graph edge-induced by $F$.

For simplicity of description, we shall use some terminologies for referring to edge subsets which are conventionally used for subgraphs, as follows. Let $F \subseteq E$. $F$ is called connected if $G[F]$ is connected. A connected component of $F$ is the edge set of a connected component of $G[F] . C(F)$ denotes the partition of $F$ into connected components of $F$, and $c(F)=|C(F)| . F$ is called a forest if it contains no cycle and called a tree if it is connected and a forest. $F$ is called a spanning tree of a graph $G=(V, E)$ if $F$ is a tree with $F \subseteq E$ and $V(F)=V$.

A graph is called simple if it contains neither a loop nor parallel edges. In a simple undirected graph, an edge between $i$ and $j$ is denoted by $\{i, j\}$. Similarly, in a directed graph, an edge directed from $i$ to $j$ is denoted by $(i, j)$. 
Throughout the paper, $\mathbb{K}$ denotes a field, which may be finite, and $\mathbb{F}$ a subfield of $\mathbb{K}$ such that $\mathbb{K}$ has transcendentals $\alpha_{1}, \ldots, \alpha_{k}$ that form an algebraically independent set over $\mathbb{F}$ for some finite $k$. Then we assume that vector space $\mathbb{F}^{d}$ is contained in $\mathbb{K}^{d}$ by extension of scalars. For a set $X \subseteq \mathbb{F}^{d}, \operatorname{dim}_{\mathbb{F}} X$ denotes the dimension of the linear subspace spanned by $X$ in $\mathbb{F}^{d}$.

For a finite set $E$ and a vector space $W$, the set of maps from $E$ to $W$ is denoted by $W^{E}$, i.e., $W^{E}=\{\psi \mid \psi: E \rightarrow W\}$.

For a group $\Gamma$ and $X \subseteq \Gamma,\langle X\rangle$ denotes the subgroup of $\Gamma$ generated by $X$.

\section{FUndamentals ON GAin GRAPHS}

In this section we shall review properties of gain graphs. See, e.g., 114,44,45] for concrete explanations on this topic. Propositions given in this section are rather straightforward, and the proofs can be found in [17.

2.1. Gain graphs. Let $G=(V, E)$ be a directed graph which may contain multiple edges and loops, and let $\Gamma$ be a group. A pair is called a $\Gamma$-gain graph $(G, \psi)$, in which each edge is associated with an element of $\Gamma$ by a gain function $\psi: E \rightarrow \Gamma$. $G$ is a directed graph, but its orientation is used only for the reference of the gain labeling. Namely, we can change orientation of each edge as we like by imposing a property to $\psi$ such that if an edge in one direction has label $g$, then it has $g^{-1}$ in the other direction. Thus, we often do not distinguish $G$ and the underlying undirected graph and use notation in the introduction, which was introduced for undirected graphs, if it is clear from the context.

A walk is a sequence $W=v_{0}, e_{1}, v_{1}, e_{2}, v_{2}, \ldots, v_{k-1}, e_{k}, v_{k}$ of vertices and edges such that $v_{i-1}$ and $v_{i}$ are endvertices of $e_{i}$ for $1 \leq i \leq k$. For two walks $W$ and $W^{\prime}$ for which the end vertex of $W$ and the starting vertex of $W^{\prime}$ coincide, the concatenation of $W$ and $W^{\prime}$ is the walk $W$ followed by $W^{\prime}$. A walk is called closed if the starting vertex and the end vertex coincide. The gain of a walk $W$ is defined as $\psi(W)=\psi\left(e_{1}\right) \cdot \psi\left(e_{2}\right) \cdots \psi\left(e_{k}\right)$ if each edge is oriented in the forward direction through $W$, and for a backward edge $e_{i}$ we replace $\psi\left(e_{i}\right)$ with $\psi\left(e_{i}\right)^{-1}$ in the formulation. Also for convenience we set $\psi(\emptyset)=1_{\Gamma}$.

Let $(G, \psi)$ be a gain graph. For $v \in V(G)$, we denote by $\pi_{1}(G, v)$ the set of closed walks starting at $v$. Similarly, for $X \subseteq E(G)$ and $v \in V(G), \pi_{1}(X, v)$ denotes the set of closed walks starting at $v$ and using only edges of $X$, where $\pi_{1}(X, v)=\emptyset$ if $v \notin V(X)$. For $X \subseteq E(G)$, the subgroup induced by $X$ relative to $v$ is defined as $\langle X\rangle_{\psi, v}=\left\{\psi(W) \mid W \in \pi_{1}(X, v)\right\}$.

Proposition 2.1. For any connected $X \subseteq E(G)$ and two vertices $u, v \in V(X)$, $\langle X\rangle_{\psi, u}$ is conjugate to $\langle X\rangle_{\psi, v}$.

2.2. Switching operations. For $v \in V(G)$ and $g \in \Gamma$, a switching at $v$ with $g$ changes the gain function $\psi$ on $E(G)$ as follows:

$$
\psi^{\prime}(e)= \begin{cases}g \cdot \psi(e) & \text { if } e \text { is directed from } v \\ \psi(e) \cdot g^{-1} & \text { if } e \text { is directed to } v, \\ \psi(e) & \text { otherwise. }\end{cases}
$$

By definition, $\psi^{\prime}(e)=g \cdot \psi(e) \cdot g^{-1}$ if $e$ is a loop attached at $v$. We say that a gain function $\psi^{\prime}$ on $E(G)$ is equivalent to another gain function $\psi$ on $E(G)$ if $\psi^{\prime}$ is obtained from $\psi$ by a sequence of switchings. 
Proposition 2.2. Let $(G, \psi)$ be a gain graph. Let $\psi^{\prime}$ be a gain function equivalent to $\psi$. Then, for any $X \subseteq E(G)$ and any $v \in V(G),\langle X\rangle_{\psi^{\prime}, v}$ is conjugate to $\langle X\rangle_{\psi, v}$.

Proposition 2.3. Let $(G, \psi)$ be a gain graph. Then, for any forest $F \subseteq E(G)$, there is an equivalent gain function $\psi^{\prime}$ on $E(G)$ such that $\psi^{\prime}(e)$ is the identity for every $e \in F$.

Proposition 2.3 suggests a simple way to compute $\langle F\rangle_{\psi, v}$ up to congruence, in analogy with the fact that a cycle space of a graph is spanned by fundamental cycles. For a connected $X \subseteq E(G)$, take a spanning tree $T$ of $G[X]$. By Proposition 2.3 we can convert the gain function to an equivalent gain function $\phi$ such that $\phi(e)=1_{\Gamma}$ for all $e \in T$. Then, observe that any closed walk $W \in \pi_{1}(X, v)$ can be considered as concatenations of closed walks $W_{1}, W_{2}, \ldots, W_{k}$ such that $W_{i}$ is a closed walk in $\pi_{1}(X, v)$ that passes through only one edge of $X \backslash T$. Since $\phi(e)$ is the identity for all $e \in T$, it follows that $\phi(W)$ is a product of elements in $\{\phi(e): e \in X \backslash T\}$, implying that $\langle X\rangle_{\phi, v} \subseteq\langle\phi(e): e \in X \backslash T\rangle$. Conversely, $\phi(e)$ is contained in $\langle X\rangle_{\phi, v}$ for all $e \in X \backslash T$. Thus, $\langle X\rangle_{\phi, v}=\langle\phi(e): e \in X \backslash T\rangle$. In particular, we proved the following.

Proposition 2.4. For a connected $X \subseteq E(G)$ and a spanning tree $T$ of $G[X]$, suppose that $\psi(e)$ is the identity for all $e \in T$. Then, $\langle X\rangle_{\psi, v}=\langle\psi(e): e \in X \backslash T\rangle$.

A connected edge subset $F$ in a gain graph $(G, \psi)$ is called balanced if $\langle F\rangle_{\psi, v}$ is the identity group for some $v \in V(F)$. F is called unbalanced if it is not balanced. By Proposition 2.1, this property is invariant under the choice of the base vertex $v \in V(F)$, and $F$ is unbalanced if and only if $F$ contains an unbalanced cycle. Thus, we can extend this notion to any $F \subseteq E(G)$ (possibly disconnected sets) such that $F$ is unbalanced if and only if $F$ contains an unbalanced cycle.

\section{Matroids AND POLYMATROIDS}

3.1. Matroids and polymatroids induced by submodular functions. Let $E$ be a finite set. A function $\mu: 2^{E} \rightarrow \mathbb{R}$ is called submodular if $\mu(X)+\mu(Y) \geq$ $\mu(X \cup Y)+\mu(X \cap Y)$ for every $X, Y \subseteq E$. It is well known that $\mu: 2^{E} \rightarrow \mathbb{R}$ is submodular if and only if $\mu(X \cup\{e\})-\mu(X) \geq \mu(Y \cup\{e\})-\mu(Y)$ for any $X \subseteq Y \subseteq E$ and $e \in E \backslash Y$. $\mu$ is called monotone if $\mu(X) \leq \mu(Y)$ for any $X \subseteq Y$. $\mu$ is called normalized if $\mu(\emptyset)=0$.

Edmonds 9 observed that an integer-valued monotone submodular function $\mu: 2^{E} \rightarrow \mathbb{Z}$ induces a matroid on $E$, denoted by $\mathbf{M}(\mu)$, where $F \subseteq E$ is independent if and only if $|X| \leq \mu(X)$ for every nonempty $X \subseteq F$. This matroid can be understood through the following constructions of set functions, known as Dilworth truncation and restriction.

Given an integer-valued function $\mu: 2^{E} \rightarrow \mathbb{Z}$, we define the Dilworth truncation $\hat{\mu}: 2^{E} \rightarrow \mathbb{R}$ by

$$
\hat{\mu}(F)=\min \left\{\sum_{1 \leq i \leq k} \mu\left(F_{i}\right) \mid \text { a partition }\left\{F_{1}, \ldots, F_{k}\right\} \text { of } F_{+}\right\} \quad(F \subseteq E)
$$

where $F_{+}=\{e \in F \mid \mu(e) \geq 0\}$, and $\hat{\mu}(F)=0$ if $F_{+}=\emptyset$. We define the restriction $\mu^{\mathbf{1}}: 2^{E} \rightarrow \mathbb{Z}$ of $\mu$ by

$$
\mu^{\mathbf{1}}(F)=\min \{|F \backslash X|+\mu(X) \mid X \subseteq F\} \quad(F \subseteq E) .
$$


It is known that if $\mu$ is monotone submodular, then $\hat{\mu}$ and $\mu^{\mathbf{1}}$ are monotone submodular (see, e.g., [31, Section 48.2], [12, Theorem 2.6], and [12, Section 3.1(b)]). Therefore, the combination $\hat{\mu}^{\mathbf{1}}$, given by

$$
\hat{\mu}^{\mathbf{1}}(F)=\min \left\{\left|F_{+} \backslash \bigcup_{i=1}^{k} F_{i}\right|+\sum_{i=1}^{k} \mu\left(F_{i}\right) \mid \text { a subpartition }\left\{F_{1}, \ldots, F_{k}\right\} \text { of } F_{+}\right\}
$$

for each $F \subseteq E$, is a monotone submodular function. Moreover $\hat{\mu}^{\mathbf{1}}(\emptyset)=0$ and $\hat{\mu}^{\mathbf{1}}(e) \leq 1$ for $e \in E$. This implies that $\hat{\mu}^{\mathbf{1}}$ is a rank function of a matroid on $E$. Observe that $F \subseteq E$ is independent in this matroid if and only if $|X| \leq \hat{\mu}(X)$ for any $X \subseteq F$ by (3.2). The latter condition is equivalent to $|X| \leq \mu(X)$ for any nonempty $X \subseteq F$ by (3.1). Thus $\hat{\mu}^{\mathbf{1}}$ is indeed the rank function of the matroid $\mathbf{M}(\mu)$ induced by $\mu$.

Throughout the paper a matroid is denoted by a pair $(E, \mu)$ of the ground set $E$ and the rank function $\mu$, where $\mu$ is a normalized monotone submodular function satisfying $\mu(e) \leq 1$ for all $e \in E$. A polymatroid is a generalization of matroids. A pair $(E, \mu)$ of a finite set $E$ and $\mu: 2^{E} \rightarrow \mathbb{Z}$ is said to be a polymatroid if $\mu$ is a normalized monotone submodular function (where $\mu(e)>1$ may hold for $e \in E$ ).

For a monotone submodular function $\mu: 2^{E} \rightarrow \mathbb{Z}, \hat{\mu}$ is a normalized monotone submodular function. Hence $(E, \hat{\mu})$ is a polymatroid, which is called the polymatroid induced by $\mu$, denoted by $\mathbf{P}(\mu)$.

3.2. Matroid union. Let us consider two monotone submodular functions $\mu_{1}$ and $\mu_{2}$ on a finite set $E$. Since the monotonicity and the submodularity are preserved by taking summation, $\mu_{1}+\mu_{2}$ is monotone and submodular. Thus, for two polymatroids $\mathbf{P}_{1}=\left(E, \mu_{1}\right)$ and $\mathbf{P}_{2}=\left(E, \mu_{2}\right),\left(E, \mu_{1}+\mu_{2}\right)$ forms a polymatroid, which is called the sum of $\mathbf{P}_{1}$ and $\mathbf{P}_{2}$.

In a similar manner, suppose that we have two matroids $\mathbf{M}_{1}=\left(E, r_{1}\right)$ and $\mathbf{M}_{2}=\left(E, r_{2}\right)$ with the rank functions $r_{1}$ and $r_{2}$. Their union $\mathbf{M}_{1} \vee \mathbf{M}_{2}$ is defined by $\left(E,\left(r_{1}+r_{2}\right)^{\mathbf{1}}\right)$, i.e., $\left(r_{1}+r_{2}\right)^{\mathbf{1}}(F)=\min \left\{|F \backslash X|+r_{1}(X)+r_{2}(X) \mid X \subseteq F\right\}$ for $F \subseteq E$. It is well known that $F$ is independent in $\mathbf{M}_{1} \vee \mathbf{M}_{2}$ if and only if $F$ can be partitioned into $F_{1}$ and $F_{2}$ such that $F_{i}$ is independent in $\mathbf{M}_{i}$ for $i=1,2[8]$.

3.3. Linear polymatroids. Let $\mathbb{K}$ be a field and $\mathbb{F}$ be a subfield of $\mathbb{K}$ as defined in the introduction. For a finite set $E$, let us associate a linear subspace $A_{e}$ of $\mathbb{F}^{d}$ with each $e \in E$ by $\Phi: e \in E \mapsto A_{e} \subseteq \mathbb{F}^{d}$. Then, $\operatorname{dim}_{\Phi}: 2^{E} \rightarrow \mathbb{Z}$, defined by $\operatorname{dim}_{\Phi}(F)=\operatorname{dim}_{\mathbb{F}}\left\{A_{e} \mid e \in F\right\}$, is a set function on $E$, and $\left(E, \operatorname{dim}_{\Phi}\right)$ forms a polymatroid, denoted by $\mathbf{L P}(E, \Phi)$. If a polymatroid $(E, \mu)$ is isomorphic to $\mathbf{L P}(E, \Phi)$ for some $\Phi$ (i.e., $\mu(F)=\operatorname{dim}_{\Phi}(F)$ for any $\left.F \subseteq E\right),(E, \mu)$ is said to be a linear polymatroid, and $\Phi$ is called a linear representation of $(E, \mu)$.

If $(E, \mu)$ is a matroid, a linear representation $\Phi$ is sometimes referred to as an assignment of a vector rather than a 1-dimensional linear space, with each element in $E$.

3.3.1. Generic linear matroids. In $\$ 3.1$ we have reviewed two operations, restrictions and Dilworth truncations. Below, we shall take a look at geometric interpretations of these operations for linear polymatroids.

Let $\mathbf{L P}(E, \Phi)$ be a linear polymatroid with a linear representation $\Phi: e \in$ $E \mapsto A_{e} \subseteq \mathbb{F}^{d}$. For each $e \in E$, we shall pick a base $v_{1}, \ldots, v_{k_{e}}$ of $A_{e}$, where $k_{e}=\operatorname{dim}_{\mathbb{F}} A_{e}$, and define a representative vector by $x_{e}=\sum_{i} \alpha_{e}^{i} v_{i}$, where $\alpha_{e}^{i}$ is a number in $\mathbb{K}$ such that $\left\{\alpha_{e}^{i}: e \in E, 1 \leq i \leq k_{e}\right\}$ is algebraically independent over $\mathbb{F}$. 
That is, by extending the underlying field from $\mathbb{F}$ to $\mathbb{K}$, we have generically chosen a representative vector $x_{e}$ from each $A_{e}$.

This gives us a linear matroid with a linear representation $e \mapsto x_{e}$ over $\mathbb{K}$. Lovász [19] gave its rank formula.

Theorem 3.1 (Lovász [19]). Let $\mathbb{K}$ be a field and $\mathbb{F}$ be a subfield of $\mathbb{K}$. Let $\mathbf{L P}(E, \Phi)$ be a linear polymatroid with a linear representation $\Phi: e \in E \mapsto A_{e} \subseteq \mathbb{F}^{d}$, and suppose that a representative vector $x_{e}$ is generically chosen from each $A_{e}$ over $\mathbb{K}$. Then,

$$
\operatorname{dim}_{\mathbb{K}}\left\{x_{e} \mid e \in E\right\}=\min \left\{|E \backslash F|+\operatorname{dim}_{\mathbb{F}}\left\{A_{e} \mid e \in F\right\} \mid F \subseteq E\right\} .
$$

Note that the right hand side of (3.4) does not rely on the choice of representative vectors, and hence this motivates us to define the generic matroid. The generic matroid obtained from $\mathbf{L P}(E, \Phi)$, denoted by $\mathbf{L M}(E, \Phi)$, is defined to be a matroid with a linear representation $e \mapsto x_{e}$ over $\mathbb{K}$. Notice the coincidence of two formulas (3.2) and (3.4); namely, taking the generic matroid has the same meaning as the restriction for linear polymatroids.

Lovász actually proved Theorem 3.1 under a much weaker assumption. For a family $\left\{A_{e} \mid e \in E\right\}$ of linear subspaces in $\mathbb{K}^{d}$, a set of vectors $x_{e}$ taken from each $A_{e}$ is said to be in generic position if

$$
\text { 5) } x_{f} \in \operatorname{span}\left\{x_{e} \mid e \in X\right\} \Rightarrow A_{f} \subseteq \operatorname{span}\left\{x_{e} \mid e \in X\right\} \quad \forall X \subseteq E, \forall f \in E \backslash X .
$$

It is known that (3.4) holds if $\left\{x_{e} \mid e \in E\right\}$ is in generic position [19].

3.3.2. Dilworth truncation. We also have a geometric interpretation of Dilworth truncation. For a linear polymatroid $\mathbf{L P}(E, \Phi)$ with $\Phi: e \mapsto A_{e}$, let $\mathcal{A}=\left\{A_{e} \mid\right.$ $e \in E\}$. We now consider restricting $\mathcal{A}$ to a generic hyperplane (i.e., a $d-1$ dimensional linear subspace) by extending the underlying field $\mathbb{F}$ to $\mathbb{K}$, again. A hyperplane $H$ is called generic if it is expressed by $H=\left\{x \in \mathbb{K}^{d} \mid \sum_{1 \leq i \leq d} \alpha_{i} x(i)=\right.$ $0\}$ for some algebraically independent numbers $\left\{\alpha_{1}, \ldots, \alpha_{d}\right\}$ over $\mathbb{F}$. Lovász [19] observed the following formula.

Theorem 3.2 (Lovász [19]). Let $\mathbb{K}$ be a field and $\mathbb{F}$ a subfield of $\mathbb{K}$. Let $\mathbf{L P}(E, \Phi)$ be a linear polymatroid with a linear representation $\Phi: e \in E \mapsto A_{e} \subseteq \mathbb{F}^{d}$, and let $H$ be a generic hyperplane of $\mathbb{K}^{d}$. Then,

$$
\operatorname{dim}_{\mathbb{K}}\left\{A_{e} \cap H \mid e \in E\right\}=\min \left\{\sum_{i=1}^{k}\left(\operatorname{dim}_{\mathbb{F}}\left\{A_{e} \mid e \in E_{i}\right\}-1\right)\right\},
$$

where the minimum is taken over all partitions $\left\{E_{1}, \ldots, E_{k}\right\}$ of $E$ into nonempty subsets.

The same result was also obtained by Mason [23, 24] from the viewpoint of combinatorial geometry (projective matroids).

Setting $\mu(F)=\operatorname{dim}_{\mathbb{K}}\left\{A_{e} \mid e \in F\right\}-1$ for $F \subseteq E$, we see that the polymatroid induced by $\mu$, that is, $(E, \hat{\mu})$, has linear representation $e \mapsto A_{e} \cap H$ from the coincidence of (3.1) and (3.6).

3.3.3. Linear matroid union. A linear representation of the sum of two polymatroids can easily be obtained in the following manner. Suppose that we have two linear polymatroids $(E, \mu)$ and $\left(E, \mu^{\prime}\right)$ with linear representations $\Phi: e \in E \mapsto$ $A_{e} \subseteq \mathbb{F}^{s}$ and $\Phi^{\prime}: e \in E \mapsto A_{e}^{\prime} \subseteq \mathbb{F}^{t}$, respectively. By definition, $\left(\mu+\mu^{\prime}\right)(F)=$ $\mu(F)+\mu^{\prime}(F)=\operatorname{dim}_{\mathbb{F}}\left\{A_{e} \mid e \in F\right\}+\operatorname{dim}_{\mathbb{F}}\left\{A_{e}^{\prime} \mid e \in F\right\}$. Hence, if we prepare 
$\mathbb{F}^{s+t}$ as the underlying vector space, the polymatroid $\left(E, \mu+\mu^{\prime}\right)$ is represented by $e \mapsto A_{e} \oplus A_{e}^{\prime}$.

Combining this with the discussions of 33.2 and 3.3 .1 , it is now straightforward to see the following.

Proposition 3.3. Let $\mathbf{M}_{i}$ be a matroid on a finite set $E$ with a linear representation $e \mapsto x_{e}^{i}$ in a vector space $W_{i}$ for each $i=1,2$. Then, $\mathbf{M}_{1} \vee \mathbf{M}_{2}$ is represented by $e \mapsto x_{e}$, where $x_{e}$ is a representative vector taken from $\operatorname{span}\left\{x_{e}^{1}\right\} \oplus \operatorname{span}\left\{x_{e}^{2}\right\} \subseteq$ $W_{1} \oplus W_{2}$ in generic position.

This fact is at least known from [23].

\section{Matroids induCED By SUbmodular FunCtions OVER GROUPS}

4.1. Frame matroids. Let $\Theta$ be the graph with two vertices $u$ and $v$ and three parallel edges. A subdivision of $\Theta$ is called a theta graph. Hence, a theta graph consists of three openly disjoint paths between $u$ and $v$ and contains three cycles.

Consider an undirected multigraph, which may contain loops and parallel edges. A family $\mathcal{C}$ of cycles is called a linear class if it satisfies the following property. If two cycles in $\mathcal{C}$ form a theta subgraph, then the third cycle of the theta subgraph is also contained in $\mathcal{C}$. For a graph $G=(V, E)$ and a linear class $\mathcal{C}$ of cycles, the frame matroid $\mathbf{F}(G, \mathcal{C})$ is defined such that $F \subseteq E$ is independent if and only if each connected component of $F$ contains no cycle or just one cycle, which is not included in the linear class $\mathcal{C}$ 44, 45. Therefore, the rank of $F \subseteq E$ in $\mathbf{F}(G, \mathcal{C})$ is equal to

$$
g_{\mathcal{C}}(F):=|V(F)|-c(F)+\sum_{X \in C(F)} \alpha_{\mathcal{C}}(X) \quad(F \subseteq E)
$$

where

$$
\alpha_{\mathcal{C}}(X)= \begin{cases}1 & \text { if } X \text { contains a cycle not included in } \mathcal{C} \\ 0 & \text { otherwise }\end{cases}
$$

This also implies that $g_{\mathcal{C}}$ is monotone and submodular.

In this paper we are interested in frame matroids on gain graphs. Let $(G=$ $(V, E), \psi)$ be a $\Gamma$-gain graph for a group $\Gamma$. Let $\mathcal{C}$ be the set of balanced cycles in $(G, \psi)$. Then, $\mathcal{C}$ forms a linear class, and the associated frame matroid is defined. This matroid is called the frame matroid of $(G, \psi)$, denoted by $\mathbf{F}(G, \psi)$. If we define $g_{\Gamma}: 2^{E} \rightarrow \mathbb{Z}$ by

$$
g_{\Gamma}(F)=|V(F)|-c(F)+\sum_{X \in C(F)} \alpha_{\Gamma}(X) \quad(F \subseteq E)
$$

where

$$
\alpha_{\Gamma}(X)= \begin{cases}1 & \text { if } X \text { is unbalanced } \\ 0 & \text { otherwise }\end{cases}
$$

then $g_{\Gamma}$ is the rank function of $\mathbf{F}(G, \psi)$.

4.2. Lifting based on submodular functions on groups. We now extend the construction of the union of frame matroids by using structures of the underlying group. The idea is to replace the term $\alpha_{\Gamma}$ by a function taking fractional values. 
For a group $\Gamma$, we consider a function $\mu: 2^{\Gamma} \rightarrow \mathbb{R}$ satisfying the following properties:

(Normalized) $\mu(\emptyset)=0$;

(Monotonicity) $\mu(X) \leq \mu(Y)$ for any $X \subseteq Y \subseteq \Gamma$;

(Submodularity) $\mu(X)+\mu(Y) \geq \mu(X \cup Y)+\mu(X \cap Y)$ for any $X, Y \subseteq \Gamma$;

(Invariance under closure) $\mu(X)=\mu(\langle X\rangle)$ for any nonempty $X \subseteq \Gamma$;

(Invariance under conjugation) $\mu(X)=\mu\left(\gamma X \gamma^{-1}\right)$ for any nonempty $X \subseteq \Gamma$ and $\gamma \in \Gamma$.

We say that $\mu: 2^{\Gamma} \rightarrow \mathbb{R}_{+}$is a symmetric polymatroidal function over $\Gamma$ if $\mu$ satisfies these five conditions. The submodularity implies that, for any $X \subseteq Y \subseteq \Gamma$ and $e \in \Gamma$,

$$
\mu(X \cup\{e\})-\mu(X) \geq \mu(Y \cup\{e\})-\mu(Y) .
$$

Extending the rank function (4.1) of frame matroids, we now propose a submodular function based on a symmetric polymatroidal function $\mu$. Let $(G=(V, E), \psi)$ be a $\Gamma$-gain graph. We consider $\mu\left(\langle F\rangle_{\psi, v}\right)$ for a connected $F \subseteq E$ and $v \in V(F)$. By Proposition 2.1. $\langle F\rangle_{\psi, v}$ is conjugate to $\langle F\rangle_{\psi, u}$ for any $u, v \in V(F)$ for $F \subseteq E$, and hence $\mu\left(\langle F\rangle_{\psi, u}\right)=\mu\left(\langle F\rangle_{\psi, v}\right)$ for any $u, v \in V(F)$. Also, by Proposition 2.2, $\mu\left(\langle F\rangle_{\psi, v}\right)$ is invariant with respect to the choice of equivalent gain functions $\psi$. We hence simply denote $\mu\left(\langle F\rangle_{\psi, v}\right)$ by $\mu\langle F\rangle$, implicitly assuming the gain function and the base vertex among $V(F)$. We can then define a set function $g_{\mu}: 2^{E} \rightarrow \mathbb{R}$ by

$$
g_{\mu}(F)=|V(F)|-c(F)+\sum_{X \in C(F)} \mu\langle X\rangle \quad(F \subseteq E) .
$$

Notice that if $X$ and $Y$ are connected with $X \subseteq Y \subseteq E$, we have $\mu\langle X\rangle \leq \mu\langle Y\rangle$ by the monotonicity of $\mu$ over $\Gamma$. However, the monotonicity and the submodularity of $\mu$ do not hold over $E$ in general. The next theorem ensures these properties for $g_{\mu}$.

Theorem 4.1. Let $\mu: 2^{\Gamma} \rightarrow[0,1]$ be a symmetric polymatroidal function over a group $\Gamma$ (with the upper bound 1 ) and $(G=(V, E), \psi)$ a $\Gamma$-gain graph. Then, $g_{\mu}$ is a monotone submodular function over $E$.

Proof. For each $X \subseteq E$ and $e=(i, j) \in E \backslash X$, let $\Delta(X, e)=g_{\mu}(X \cup\{e\})-g_{\mu}(X)$. We denote by $X_{i}$ the connected component of $X$ for which $i \in V\left(X_{i}\right)$. If such a component does not exist, let $X_{i}=\emptyset$. Similarly, we denote by $X_{j}$ the component of $X$ for which $j \in V\left(X_{j}\right)$.

By a simple calculation, we have the following relation:

$\Delta(X, e)= \begin{cases}\mu\left\langle X_{i} \cup\{e\}\right\rangle-\mu\left\langle X_{i}\right\rangle & \text { if } e \text { is a loop or } X_{i}=X_{j} \neq \emptyset, \\ \mu\left\langle X_{i} \cup X_{j} \cup\{e\}\right\rangle+1-\mu\left\langle X_{i}\right\rangle-\mu\left\langle X_{j}\right\rangle & \text { otherwise. }\end{cases}$

Let us check the monotonicity of $g_{\mu}$. If $e$ is a loop or $X_{i}=X_{j} \neq \emptyset$, then $\mu\left\langle X_{i} \cup\{e\}\right\rangle-\mu\left\langle X_{i}\right\rangle \geq 0$ by the monotonicity of $\mu$ over $\Gamma$. Otherwise, since $X_{i}$ and $X_{i} \cup X_{j} \cup\{e\}$ are connected, we have $\mu\left\langle X_{i}\right\rangle \leq \mu\left\langle X_{i} \cup X_{j} \cup\{e\}\right\rangle$ by the monotonicity of $\mu$ over $\Gamma$. Also, by the upper bound of $\mu, \mu\left\langle X_{j}\right\rangle \leq 1$. We thus have $\Delta(X, e)=\mu\left\langle X_{i} \cup X_{j} \cup\{e\}\right\rangle+1-\left(\mu\left\langle X_{i}\right\rangle+\mu\left\langle X_{j}\right\rangle\right) \geq 0$. This completes the proof of the monotonicity. 
For the submodularity, we check

$$
\Delta(X, e) \geq \Delta(Y, e)
$$

for any $X \subseteq Y \subseteq E$ and $e \in E \backslash Y$. We split the proof into two cases.

Case 1. Suppose that $e$ is a loop or $X_{i}=X_{j} \neq \emptyset$. We then have $X_{i} \subseteq Y_{i}=Y_{j}$. We take a tree $T \subseteq Y_{i}$ spanning $V\left(Y_{i}\right)$ such that $T \cap X_{i}$ forms a tree spanning $V\left(X_{i}\right)$. By using switching operations, we may assume by Proposition 2.3 that $\psi(f)=1_{\Gamma}$ for every $f \in T$. Observe then that $\left\langle Y_{i} \cup\{e\}\right\rangle_{\psi, i}=\left\langle\left\langle Y_{i}\right\rangle_{\psi, i} \cup\{\psi(e)\}\right\rangle$ and $\left\langle X_{i} \cup\{e\}\right\rangle_{\psi, i}=\left\langle\left\langle X_{i}\right\rangle_{\psi, i} \cup\{\psi(e)\}\right\rangle$ by Proposition 2.4. We thus have

$$
\begin{aligned}
\Delta(X, e) & =\mu\left\langle X_{i} \cup\{e\}\right\rangle-\mu\left\langle X_{i}\right\rangle \\
& =\mu\left(\left\langle\left\langle X_{i}\right\rangle_{\psi, i} \cup\{\psi(e)\}\right\rangle\right)-\mu\left(\left\langle X_{i}\right\rangle_{\psi, i}\right) \\
& =\mu\left(\left\langle X_{i}\right\rangle_{\psi, i} \cup\{\psi(e)\}\right)-\mu\left(\left\langle X_{i}\right\rangle_{\psi, i}\right) \\
& \geq \mu\left(\left\langle Y_{i}\right\rangle_{\psi, i} \cup\{\psi(e)\}\right)-\mu\left(\left\langle Y_{i}\right\rangle_{\psi, i}\right) \\
& =\mu\left(\left\langle\left\langle Y_{i}\right\rangle_{\psi, i} \cup\{\psi(e)\}\right\rangle\right)-\mu\left(\left\langle Y_{i}\right\rangle_{\psi, i}\right) \\
& =\mu\left\langle Y_{i} \cup\{e\}\right\rangle-\mu\left\langle Y_{i}\right\rangle=\Delta(Y, e),
\end{aligned}
$$

where we used (4.3), (4.5) and the invariance of $\mu$ under closures.

Case 2. Suppose that $e$ is a nonloop edge and at least one of $X_{i} \neq X_{j}$ or $X_{i}=$ $X_{j}=\emptyset$ holds. We further split the proof into subcases.

(2-i) If $Y_{i}=Y_{j} \neq \emptyset$, then, by (4.5), we have

$$
\Delta(X, e)-\Delta(Y, e)=\mu\left\langle X_{i} \cup X_{j} \cup\{e\}\right\rangle+1+\mu\left\langle Y_{i}\right\rangle-\mu\left\langle X_{i}\right\rangle-\mu\left\langle X_{j}\right\rangle-\mu\left\langle Y_{i} \cup\{e\}\right\rangle .
$$

Since all these sets are connected or empty, $\mu\left\langle X_{i} \cup X_{j} \cup\{e\}\right\rangle \geq \mu\left\langle X_{j}\right\rangle, \mu\left\langle Y_{i}\right\rangle \geq \mu\left\langle X_{i}\right\rangle$, and $1 \geq \mu\left\langle Y_{i} \cup\{e\}\right\rangle$. Thus, $\mu\left\langle X_{i} \cup X_{j} \cup\{e\}\right\rangle+\mu\left\langle Y_{i}\right\rangle+1 \geq \mu\left\langle X_{i}\right\rangle+\mu\left\langle X_{j}\right\rangle+\mu\left\langle Y_{i} \cup\{e\}\right\rangle$, implying (4.6).

(2-ii) If $Y_{i} \neq Y_{j}$ or $Y_{i}=Y_{j}=\emptyset$ holds, then $e$ is a bridge connecting $X_{i}$ and $X_{j}$ in $X_{i} \cup X_{j} \cup\{e\}$ and is also a bridge connecting $Y_{i}$ and $Y_{j}$ in $Y_{i} \cup Y_{j} \cup\{e\}$. By a switch operation, we may assume that $\psi(e)$ is the identity. Then, $\left\langle X_{i} \cup X_{j} \cup\{e\}\right\rangle_{\psi, i}=$ $\left\langle\left\langle X_{i}\right\rangle_{\psi, i} \cup\left\langle X_{j}\right\rangle_{\psi, j}\right\rangle$. This implies

$$
\mu\left\langle X_{i} \cup X_{j} \cup\{e\}\right\rangle=\mu\left(\left\langle X_{i}\right\rangle_{\psi, i} \cup\left\langle X_{j}\right\rangle_{\psi, j}\right)
$$

by the invariance under closure. Symmetrically, we have

$$
\mu\left\langle Y_{i} \cup Y_{j} \cup\{e\}\right\rangle=\mu\left(\left\langle Y_{i}\right\rangle_{\psi, i} \cup\left\langle Y_{j}\right\rangle_{\psi, j}\right) .
$$

By using the submodularity and the monotonicity of $\mu$ over $\Gamma$, along with $X_{k} \subseteq Y_{k}$ for $k=1,2$, we have

$$
\begin{aligned}
& \mu\left\langle X_{i} \cup X_{j} \cup\{e\}\right\rangle+\mu\left\langle Y_{i}\right\rangle+\mu\left\langle Y_{j}\right\rangle \\
& =\mu\left(\left\langle X_{i}\right\rangle_{\psi, i} \cup\left\langle X_{j}\right\rangle_{\psi, j}\right)+\mu\left(\left\langle Y_{i}\right\rangle_{\psi, i}\right)+\mu\left(\left\langle Y_{j}\right\rangle_{\psi, j}\right) \\
& \geq \mu\left(\left\langle X_{i}\right\rangle_{\psi, i} \cup\left\langle X_{j}\right\rangle_{\psi, j} \cup\left\langle Y_{i}\right\rangle_{\psi, i}\right)+\mu\left(\left(\left\langle X_{i}\right\rangle_{\psi, i} \cup\left\langle X_{j}\right\rangle_{\psi, j}\right) \cap\left\langle Y_{i}\right\rangle_{\psi, i}\right)+\mu\left(\left\langle Y_{j}\right\rangle_{\psi, j}\right) \\
& \geq \mu\left(\left\langle Y_{i}\right\rangle_{\psi, i} \cup\left\langle X_{j}\right\rangle_{\psi, j}\right)+\mu\left(\left\langle X_{i}\right\rangle_{\psi, i}\right)+\mu\left(\left\langle Y_{j}\right\rangle_{\psi, j}\right) \\
& \geq \mu\left(\left\langle Y_{i}\right\rangle_{\psi, i} \cup\left\langle X_{j}\right\rangle_{\psi, j} \cup\left\langle Y_{j}\right\rangle_{\psi, j}\right)+\mu\left(\left(\left\langle Y_{i}\right\rangle_{\psi, i} \cup\left\langle X_{j}\right\rangle_{\psi, j}\right) \cap\left\langle Y_{j}\right\rangle_{\psi, j}\right)+\mu\left(\left\langle X_{i}\right\rangle_{\psi, i}\right) \\
& \geq \mu\left(\left\langle Y_{i}\right\rangle_{\psi, i} \cup\left\langle Y_{j}\right\rangle_{\psi, j}\right)+\mu\left(\left\langle X_{j}\right\rangle_{\psi, j}\right)+\mu\left(\left\langle X_{i}\right\rangle_{\psi, i}\right) \\
& =\mu\left\langle Y_{i} \cup Y_{j} \cup\{e\}\right\rangle+\mu\left\langle X_{j}\right\rangle+\mu\left\langle X_{i}\right\rangle .
\end{aligned}
$$

This implies (4.6) by (4.5). 
The aim of this paper is to extend the concept of the union of frame matroids. We shall thus concentrate on a function $\mu$ taking fractional values, that is, $\mu: 2^{\Gamma} \rightarrow$ $\left\{0, \frac{1}{d}, \ldots, \frac{d-1}{d}, 1\right\}$ for some finite positive integer $d$. As it is not integer-valued, $g_{\mu}$ does not induce a matroid in general, but if we define $f_{\mu}: 2^{E} \rightarrow \mathbb{Z}$ by

$$
f_{\mu}(F)=d g_{\mu}(F) \quad(F \subseteq E),
$$

then $f_{\mu}$ is a normalized integer-valued monotone submodular function by Theorem 4.1. Thus $f_{\mu}$ induces a polymatroid $\mathbf{P}\left(f_{\mu}\right)=\left(E, f_{\mu}\right)$ and a matroid $\mathbf{M}\left(f_{\mu}\right)=$ $\left(E, f_{\mu}^{\mathbf{1}}\right)$ on $E$.

Example 4.1. The frame matroid (or the union of copies) is a special case of $\mathbf{M}\left(f_{\mu}\right)$, where $\mu$ is defined by $\mu(X)=0$ for $X=\emptyset$ or $X=\left\{1_{\Gamma}\right\}$, and otherwise $\mu(X)=1$. In this case, (4.7) is equal to (4.1).

Example 4.2. Let us consider a group $\Gamma$ equipped with a linear representation $\rho: \Gamma \rightarrow G L\left(\mathbb{F}^{d}\right)$ over a field $\mathbb{F}$. Let $d_{\rho}: 2^{\Gamma} \rightarrow \mathbb{Z}$ be a function defined by

$$
d_{\rho}(X)=\operatorname{dim}_{\mathbb{F}}\left\{\operatorname{image}\left(I_{d}-\rho(\gamma)\right) \mid \gamma \in X\right\} \quad(X \subseteq \Gamma),
$$

where $d_{\rho}(\emptyset)=0, I_{d}$ denotes the identity matrix of size $d \times d$, and image $\left(I_{d}-\rho(\gamma)\right)=$ $\left\{\left(I_{d}-\rho(\gamma)\right) x \mid x \in \mathbb{F}^{d}\right\}$.

It is easy to see that $d_{\rho}$ is monotone submodular and is invariant under conjugation. Also, for any $\gamma_{1}, \gamma_{2} \in \Gamma$, we have image $\left(I_{d}-\rho\left(\gamma_{1} \gamma_{2}\right)\right) \subseteq \operatorname{image}\left(I_{d}-\rho\left(\gamma_{1}\right)\right)+$ image $\left(I_{d}-\rho\left(\gamma_{2}\right)\right)$ since $\left(I_{d}-\rho\left(\gamma_{1} \gamma_{2}\right)\right)=-\left(I_{d}-\rho\left(\gamma_{1}\right)\right)\left(I_{d}-\rho\left(\gamma_{2}\right)\right)+\left(I_{d}-\rho\left(\gamma_{1}\right)\right)+$ $\left(I_{d}-\rho\left(\gamma_{2}\right)\right)$ and image $\left(\left(I_{d}-\rho\left(\gamma_{1}\right)\right)\left(I_{d}-\rho\left(\gamma_{2}\right)\right)\right) \subseteq$ image $\left(I_{d}-\rho\left(\gamma_{1}\right)\right)$. This implies the invariance of $d_{\rho}$ under closure. Therefore, by setting $\mu=d_{\rho} / d$, we have another example of a symmetric polymatroidal function $\mu$. The corresponding matroid will be extensively discussed in the next section.

\section{MAtroids INDUCED BY GROUP REPRESENTATIONS}

Dowling geometries [7] are special cases of frame matroids for finite groups, which admit linear representations over finite fields $\mathbb{F}$. In this section, we shall extend the union of Dowling geometries based on group representations.

5.1. Dowling geometries. Suppose that $\Gamma$ is a nontrivial finite group and $n$ is a positive integer. Define a $\Gamma$-gain graph $\left(K_{n}^{\bullet}(\Gamma), \psi^{\bullet}\right)$ on $V\left(K_{n}^{\bullet}(\Gamma)\right)=\{1,2, \ldots, n\}$ such that (i) for every $i, j$ with $1 \leq i<j \leq n$ and every $\gamma \in \Gamma$, it has an edge from $i$ to $j$ with gain $\gamma$ and (ii) for each vertex $i$ and $\gamma \in \Gamma$, it has a loop attached to $i$ with gain $\gamma$. The Dowling geometry $\mathbf{D}_{n}(\Gamma)$ is defined by $\mathbf{F}\left(K_{n}^{\bullet}(\Gamma), \psi^{\bullet}\right)$, the frame matroid of $\left(K_{n}^{\bullet}(\Gamma), \psi \bullet\right)$.

A remarkable property of $\mathbf{D}_{n}(\Gamma)$ is that, for $n \geq 3, \mathbf{D}_{n}(\Gamma)$ is representable over $\mathbb{F}$ if and only if $\Gamma$ is isomorphic to a subgroup of $\mathbb{F}^{\times}$(see, e.g., 27, Theorem 6.10.10]). The proof of one direction indicates the following explicit construction of the representation.

Suppose that $\Gamma$ is isomorphic to a subgroup of $\mathbb{F}^{\times}$. For a simpler description, we assume that $\Gamma$ is itself a subgroup of $\mathbb{F}^{\times}$. With each $e=(i, j) \in E\left(K_{n}^{\bullet}(\Gamma)\right)$, we associate a vector $x_{e} \in \mathbb{F}^{V}$ defined by

$$
x_{e}(v)= \begin{cases}-\psi(e) & \text { if } v=i \\ 1 & \text { if } v=j \\ 0 & \text { otherwise }\end{cases}
$$


if $e$ is not a loop, and

$$
x_{e}(v)= \begin{cases}1 & \text { if } v=i, \\ 0 & \text { otherwise }\end{cases}
$$

if $e$ is a loop attached at $i$. These give us a linear representation of $\mathbf{D}_{n}(\Gamma)$ over $\mathbb{F}$ (see, e.g., [27, Lemma 6.10.11]), which is called the canonical representation 47]. As each $\Gamma$-gain graph $(G, \psi)$ can be considered as a subgraph of $\left(K_{n}^{\bullet}(\Gamma), \psi^{\bullet}\right)$, the restriction to $E(G)$ leads to the canonical representation of $\mathbf{F}(G, \psi)$.

Equivalently, instead of a vector assignment, we may associate a 1-dimensional linear space

$$
D_{e}=\left\{\begin{array}{l|l}
x \in \mathbb{F}^{V} & \begin{array}{l}
x(i)+\psi(e) x(j)=0 \\
x(V \backslash\{i, j\})=0
\end{array}
\end{array}\right\}
$$

with each nonloop edge $e=(i, j)$, and

$$
D_{e}=\left\{x \in \mathbb{F}^{V} \mid x(V \backslash\{i\})=0\right\}
$$

with a loop $e$ attached at $i$, where, for $W \subseteq V, x(W)=0$ implies $x(k)=0$ for all $k \in W$. Then, the union of $d$ copies of $\mathbf{D}_{n}(\Gamma)$ can be obtained in a systematic manner by just following the technique mentioned in 3.3 .3 .

To see more detail, let us consider the direct sum of $d$ copies of $\mathbb{F}^{V}$, which results in $\left(\mathbb{F}^{d}\right)^{V}$. Then, the associated vector space with each edge $e=(i, j)$ becomes a $d$-dimensional space in $\left(\mathbb{F}^{d}\right)^{V}$ written by

$$
D_{e}^{d}=\left\{\begin{array}{l|l}
x \in\left(\mathbb{F}^{d}\right)^{V} & \begin{array}{l}
x(i)+\psi(e) x(j)=0, \\
x(V \backslash\{i, j\})=0
\end{array}
\end{array}\right\}
$$

and

$$
D_{e}^{d}=\left\{x \in\left(\mathbb{F}^{d}\right)^{V} \mid x(V \backslash\{i\})=0\right\} .
$$

By extending the underlying field from $\mathbb{F}$ to $\mathbb{K}$, we assume that $D_{e}^{d}$ is contained in $\mathbb{K}^{d}$ and we can take a representative vector $x_{e}^{d}$ from each $D_{e}^{d}$ in generic position. By Proposition 3.3, we obtain a linear representation $e=(i, j) \mapsto x_{e}^{d} \in\left(\mathbb{K}^{d}\right)^{V}$ of the union of $d$ copies of $\mathbf{D}_{n}(\Gamma)$, where

$$
\begin{gathered}
x_{e}^{d}(v)= \begin{cases}-\psi(e) \alpha_{e} & \text { if } v=i, \\
\alpha_{e} & \text { if } v=j, \\
0 & \text { otherwise, }\end{cases} \\
x_{e}^{d}(v)= \begin{cases}\alpha_{e} & \text { if } v=i, \\
0 & \text { otherwise }\end{cases}
\end{gathered}
$$

depending on whether $e=(i, j)$ is a nonloop or a loop, where $\alpha_{e}=\left(\alpha_{e}^{1}, \ldots, \alpha_{e}^{d}\right)^{\top} \in$ $\mathbb{K}^{d}$ such that $\left\{\alpha_{e}^{i}: 1 \leq i \leq d, e \in E\left(K_{n}^{\bullet}(\Gamma)\right)\right\}$ is algebraically independent over $\mathbb{F}$. We shall extend this construction in the next subsection.

Consider a $\Gamma$-gain graph $(G, \psi)$ such that $\psi(e)$ is the identity for every $e \in$ $E$. Then, $\mathbf{F}(G, \psi)$ is just the graphic matroid of $G$. The above result on linear representations of frame matroids implicitly implies the following fact on the linear representation of the sum of $d$ copies of the graphic matroid, which will be frequently used in the subsequent discussion. 
Lemma 5.1. Let $(G=(V, E), \psi)$ be a gain graph such that $\psi(e)$ is the identity for every $e \in E$. Suppose that $E$ is connected. Then, the following hold.

- $\operatorname{dim}_{\mathbb{F}}\left\{D_{e}^{d} \mid e \in E\right\}=d|V|-d$.

- For any $x \in\left(\mathbb{F}^{d}\right)^{V}$ with $x(V \backslash\{i, j\})=0, x \in \operatorname{span}\left\{D_{e}^{d} \mid e \in E\right\}$ if and only if $x(i)+x(j)=0$.

Proof. We may assume that $\Gamma$ is a subgroup of $\mathbb{F}^{\times}$. The first part directly follows from the above discussion on frame matroids and the canonical linear representation. Indeed, since $D_{e}^{d}$ is the direct sum of $d$ copies of $D_{e}$, we have $\operatorname{dim}_{\mathbb{F}}\left\{D_{e}^{d} \mid\right.$ $e \in E\}=d \operatorname{dim}_{\mathbb{F}}\left\{D_{e} \mid e \in E\right\}=d\left(|V|-c(E)+\alpha_{\Gamma}(E)\right)=d|V|-d$.

The second part is also implicit in the above discussion. Let us first consider the case of $d=1$. Let $e^{*}=(i, j)$ be a new edge from $i$ to $j$ with the gain $\psi\left(e^{*}\right)=g$ for some $g \in \mathbb{F} \backslash\{0\}$, and let $\left(G^{*}, \psi\right)$ be the gain graph obtained from $(G, \psi)$ by adding $e^{*}$. In the canonical representation of $\mathbf{F}\left(G^{*}, \psi\right), e^{*}$ is associated with a vector $x_{e^{*}} \in \mathbb{F}^{V}$ with $x_{e^{*}}(V \backslash\{i, j\})=0, x_{e^{*}}(j)=1$ and $x_{e^{*}}(i)=-g$. Take a spanning tree $T$ of $G$, and consider a $|V| \times|V|$ matrix whose columns are $x_{e}$ for $e \in T \cup\left\{e^{*}\right\}$. By computing its determinant explicitly, one can easily check that the matrix has rank $|V|$ if and only if $g \neq 1$. In other words $\operatorname{span}\left\{D_{e} \mid e \in E\right\}$ contains $x \in \mathbb{F}^{V} \backslash\{0\}$ with $x(V \backslash\{i, j\})=0$ if and only if $x(i)+x(j)=0$.

Thus, if we consider the direct sum of $d$ copies of $\mathbb{F}^{d}$, we conclude that $\operatorname{span}\left\{D_{e}^{d} \mid\right.$ $e \in E\}$ contains $x \in\left(\mathbb{F}^{d}\right)^{V} \backslash\{0\}$ with $x(V \backslash\{i, j\})=0$ if and only if $x(i)+x(j)=$ 0 .

5.2. Linear matroids induced by group representations. In this section, we shall extend the representation theory of the union of Dowling geometries. The idea of our construction is that, instead of coefficients $\psi(e) \in \mathbb{F}^{\times}$of $\alpha_{e}$ in (5.5), we shall make use of linear representations of groups. We then have a linear matroid induced by a group $\Gamma$, where $\Gamma$ is not restricted to finite abelian groups. We show that resulting linear matroids are special cases of matroids given in $\$ 4$

Let $\Gamma$ be a group equipped with a linear representation $\rho: \Gamma \rightarrow G L\left(\mathbb{F}^{d}\right)$ on a vector space of finite dimension $d$ over a field $\mathbb{F}$. Let $(G=(V, E), \psi)$ be a finite $\Gamma$-gain graph.

As in the previous subsection, let $\mathbb{K}$ be an extension of $\mathbb{F}$ that contains an algebraically independent set $\left\{\alpha_{e}^{i} \mid i=1, \ldots, d, e \in E\right\}$ over $\mathbb{F}$, and let $\alpha_{e}=$ $\left(\alpha_{e}^{1}, \ldots, \alpha_{e}^{d}\right)^{\top} \in \mathbb{K}^{d}$.

With each $e=(i, j) \in E$, we assign a vector $x_{e, \psi} \in\left(\mathbb{K}^{d}\right)^{V}$ defined by

$$
x_{e, \psi}(v)= \begin{cases}-\rho(\psi(e)) \alpha_{e} & \text { if } v=i \\ \alpha_{e} & \text { if } v=j \\ 0 & \text { otherwise }\end{cases}
$$

if $e=(i, j)$ is not a loop, and

$$
x_{e, \psi}(v)= \begin{cases}\left(I_{d}-\rho(\psi(e))\right) \alpha_{e} & \text { if } v=i \\ 0 & \text { otherwise }\end{cases}
$$

if $e$ is a loop. The linear matroid induced on $\left\{x_{e, \psi} \mid e \in E\right\}$ is denoted by $\mathbf{D}_{\rho}(G, \psi)$. 
Note that $\mathbf{D}_{\rho}(G, \psi)$ is the generic matroid obtained from a linear polymatroid with a linear representation $e \mapsto A_{e, \psi}$ defined by

$$
A_{e, \psi}=\left\{\begin{array}{l|l}
x \in\left(\mathbb{F}^{d}\right)^{V} & \begin{array}{l}
x(i)+\rho(\psi(e)) x(j)=0 \\
x(V \backslash\{i, j\})=0
\end{array}
\end{array}\right\}
$$

for each nonloop edge $e \in E$, and

$$
A_{e, \psi}=\left\{x \in\left(\mathbb{F}^{d}\right)^{V} \mid \exists \alpha \in \mathbb{F}^{d}: \begin{array}{l}
x(i)=\left(I_{d}-\rho(\psi(e))\right) \alpha \\
x(V \backslash\{i\})=0
\end{array}\right\}
$$

for a loop $e$, by extending the underlying field from $\mathbb{F}$ to $\mathbb{K}$. Note also that $A_{e, \psi}$ is invariant with the choice of orientation of each edge, as each $\rho(\psi(e))$ is invertible.

Let $\mathbf{D P}_{\rho}(G, \psi)$ be the linear polymatroid on $E$ represented by $e \mapsto A_{e, \psi}$. Clearly, each $A_{e, \psi}$ depends on the gain function $\psi$, but, as shown below, the associated polymatroid is actually invariant up to equivalence.

Lemma 5.2. Let $\psi$ and $\psi^{\prime}$ be equivalent gain functions. Then,

$$
\operatorname{dim}_{\mathbb{F}}\left\{A_{e, \psi} \mid e \in E\right\}=\operatorname{dim}_{\mathbb{F}}\left\{A_{e, \psi^{\prime}} \mid e \in E\right\} .
$$

Proof. Let us simply denote $d_{\psi}=\operatorname{dim}_{\mathbb{F}}\left\{A_{e, \psi} \mid e \in E\right\}$. It is sufficient to show that $d_{\psi}$ is invariant from any switch operation.

Suppose that $\psi^{\prime}$ is obtained from $\psi$ by a switch operation at $v \in V$ with $\gamma \in \Gamma$. Since $A_{e, \psi}$ is invariant with the choice of the edge orientation, we may assume that all edges incident to $v$ are oriented from $v$. Then, $\psi^{\prime}(e)=\gamma \psi(e)$ if $e$ is incident to $v, \psi^{\prime}(e)=\gamma \psi(e) \gamma^{-1}$ if $e$ is a loop at $v$, and otherwise $\psi^{\prime}(e)=\psi(e)$.

Consider a bijective linear transformation $T:\left(\mathbb{F}^{d}\right)^{V} \rightarrow\left(\mathbb{F}^{d}\right)^{V}$ defined by, for each $x \in\left(\mathbb{F}^{d}\right)^{V}$,

$$
T(x)(w)= \begin{cases}x(w) & \text { if } w \in V \backslash\{v\}, \\ \rho(\gamma) x(v) & \text { if } w=v .\end{cases}
$$

We then have $x(v)=\rho(\gamma)^{-1} T(x)(v)$ and $x(w)=T(x)(w)$ for $w \in V \backslash\{v\}$. Therefore, if $e$ is a nonloop edge oriented from $v$ to a vertex $j \in V$,

$$
\begin{aligned}
T A_{e, \psi} & =\left\{T(x) \in\left(\mathbb{F}^{d}\right)^{V} \mid x(v)+\rho(\psi(e)) x(j)=0, x(V \backslash\{v, j\})=0\right\} \\
& =\left\{y \in\left(\mathbb{F}^{d}\right)^{V} \mid y(v)+\rho(\gamma \psi(e)) y(j)=0, y(V \backslash\{v, j\})=0\right\} .
\end{aligned}
$$

As $\psi^{\prime}(e)=\gamma \psi(e)$, we obtain that $T A_{e, \psi}=A_{e, \psi^{\prime}}$. Similarly, if $e$ is a loop attached to $v$,

$$
\begin{aligned}
T A_{e, \psi} & =\left\{y \in\left(\mathbb{K}^{d}\right)^{V} \mid \exists \alpha \in \mathbb{K}^{d}: \rho\left(\gamma^{-1}\right) y(v)=\left(I_{d}-\rho(\psi(e))\right) \alpha, y(V \backslash\{v\})=0\right\} \\
& =\left\{y \in\left(\mathbb{K}^{d}\right)^{V} \mid \exists \alpha \in \mathbb{K}^{d}: y(v)=\left(I_{d}-\rho\left(\gamma \psi(e) \gamma^{-1}\right)\right) \alpha, y(V \backslash\{v\})=0\right\} \\
& =A_{e, \psi^{\prime}},
\end{aligned}
$$

where $\psi^{\prime}(e)=\gamma \psi(e) \gamma^{-1}$.

If $e$ is not incident to $v$, then we clearly have $T A_{e, \psi}=A_{e, \psi}=A_{e, \psi^{\prime}}$. Thus $d_{\psi}$ is invariant from any switch operation.

5.3. Combinatorial characterization. We now show that the linear matroid $\mathbf{D}_{\rho}(G, \psi)$ is indeed equal to a special case of matroids given in 44.2 . Recall that in 4.2 (Example 4.2) $, d_{\rho}: 2^{\Gamma} \rightarrow \mathbb{Z}$ is given by

$$
d_{\rho}(X)=\operatorname{dim}_{\mathbb{F}}\left\{\operatorname{image}\left(I_{d}-\rho(\gamma)\right) \mid \gamma \in X\right\} \quad(X \subseteq \Gamma) .
$$


Let $(G=(V, E), \psi)$ be a $\Gamma$-gain graph. As a special case of $f_{\mu}$ given in (4.7), we shall define a set function $f_{\rho}$ on $E$ by

$$
f_{\rho}(F)=d|V(F)|-d c(F)+\sum_{X \in C(F)} d_{\rho}\langle X\rangle \quad(F \subseteq E) .
$$

By Theorem 4.1 $f_{\rho}$ is a normalized integer-valued monotone submodular function, and thus $\mathbf{P}\left(f_{\rho}\right)=\left(E, f_{\rho}\right)$ is a polymatroid and $\mathbf{M}\left(f_{\rho}\right)=\left(E, f_{\rho}^{\mathbf{1}}\right)$ is a matroid on $E$.

We are now ready to state our main theorems. The first theorem asserts the equivalence of $\mathbf{P}\left(f_{\rho}\right)$ and $\mathbf{D P}_{\rho}(G, \psi)$, while the second implies the equivalence of $\mathbf{M}\left(f_{\rho}\right)$ and $\mathbf{D}_{\rho}(G, \psi)$.

Theorem 5.3. Let $\Gamma$ be a group equipped with a linear representation $\rho: \Gamma \rightarrow$ $G L\left(\mathbb{F}^{d}\right)$. Let $(G=(V, E), \psi)$ be a $\Gamma$-gain graph. Then,

$$
f_{\rho}(E)=\operatorname{dim}_{\mathbb{F}}\left\{A_{e, \psi} \mid e \in E\right\} .
$$

Proof. For any $F \subseteq E$, let $A_{F}=\operatorname{span}\left\{A_{e, \psi} \mid e \in F\right\}$. By definition, it is easy to check that $A_{E}=\bigoplus_{X \in C(E)} A_{X}$. Moreover, $f_{\rho}(E)=\sum_{X \in C(E)} f_{\rho}(X)$. Hence, it suffices to show the statement when $G$ is connected.

Let $T$ be a spanning tree in $E$. By Proposition 2.3 and Lemma 5.2 , we may assume that $\psi(e)$ is the identity for $e \in T$. By Proposition 2.4. $\langle E\rangle_{\psi, v}=\langle\psi(e)|$ $e \in E \backslash T\rangle$ for any $v \in V$. Hence, as $\mu$ is invariant under taking closure, $\mu\langle E\rangle=$ $\mu(\{\psi(e) \mid e \in E \backslash T\})$. Thus,

$$
f_{\rho}(E)=d|V|-d+\operatorname{dim}_{\mathbb{F}}\left\{\operatorname{image}\left(I_{d}-\rho(\psi(e))\right) \mid e \in E \backslash T\right\} .
$$

By Lemma 5.1, we have that (i) $\operatorname{dim}_{\mathbb{F}} A_{T}=d|V|-d$ and (ii) for any $i, j \in V$ and any $x \in\left(\mathbb{F}^{d}\right)^{V}$ with $x(V \backslash\{i, j\})=0, x \in A_{T}$ holds if and only if $x(i)+x(j)=0$. This means that each quotient space $A_{e, \psi} / A_{T}$ for $e=(i, j) \in E \backslash T$ is written by

$$
\begin{aligned}
A_{e, \psi} / A_{T} & =\left\{x+A_{T} \mid \exists \alpha \in \mathbb{F}^{d}: x(j)=\left(I_{d}-\rho(\psi(e))\right) \alpha, x(V \backslash\{j\})=0\right\} \\
& =\left\{x+A_{T} \mid \exists \alpha \in \mathbb{F}^{d}: x(1)=\left(I_{d}-\rho(\psi(e))\right) \alpha, x(V \backslash\{1\})=0\right\}
\end{aligned}
$$

where 1 denotes one specific vertex in $V$. Therefore, $\operatorname{span}\left\{A_{e, \psi} / A_{T} \mid e \in E \backslash T\right\}$ is isomorphic to

$$
\operatorname{span}\left\{x \in\left(\mathbb{F}^{d}\right)^{V} \mid \exists e \in E \backslash F, \exists \alpha \in \mathbb{F}^{d}: x(1)=\left(I_{d}-\rho(\psi(e))\right) \alpha, x(V \backslash\{1\})=0\right\},
$$

which is isomorphic to $\operatorname{span}\left\{\operatorname{image}\left(I_{d}-\rho(\psi(e))\right) \mid e \in E \backslash T\right\}$. Thus, we obtain $\operatorname{dim}_{\mathbb{F}}\left\{A_{e, \psi} \mid e \in E\right\}=\operatorname{dim}_{\mathbb{F}} A_{T}+\operatorname{dim}_{\mathbb{F}}\left\{A_{e, \psi} / A_{T} \mid e \in E \backslash T\right\}=d|V|-d+$ $\operatorname{dim}_{\mathbb{F}}\left\{\operatorname{image}\left(I_{d}-\rho(\psi(e))\right) \mid e \in E \backslash T\right\}=f_{\rho}(E)$, completing the proof of Theorem 5.3

Theorem 5.4. Let $\Gamma$ be a group equipped with a linear representation $\rho: \Gamma \rightarrow$ $G L\left(\mathbb{F}^{d}\right)$. Let $(G=(V, E), \psi)$ be a $\Gamma$-gain graph. Then, $f_{\rho}^{\mathbf{1}}$ is the rank function of $\mathbf{D}_{\rho}(G, \psi)$.

Proof. By Theorem 5.3, we have $f_{\rho}(F)=\operatorname{dim}_{\mathbb{K}} \operatorname{span}\left\{A_{e, \psi} \mid e \in F\right\}$ for any $F \subseteq E$ (by restricting the statement to the graph $(V, F)$ ). Since $x_{e, \psi}$ of (5.7), (5.8) is taken from $A_{e, \psi}$ so that $\left\{x_{e, \psi} \mid e \in E\right\}$ is in generic position, Theorem 3.1 implies that the rank of $F \subseteq E$ in $\mathbf{D}_{\rho}(G)$ is equal to $\min \left\{|X|+\operatorname{dim}_{\mathbb{K}}\left\{A_{e, \psi} \mid e \in F \backslash X\right\} \mid X \subseteq F\right\}$, which is equal to $f_{\rho}^{\mathbf{1}}(F)$ by definition (3.2). 
Corollary 5.5. Let $\Gamma$ be a group equipped with a linear representation $\rho: \Gamma \rightarrow$ $G L\left(\mathbb{F}^{d}\right)$. Let $(G=(V, E), \psi)$ be a $\Gamma$-gain graph. Then, $\left\{x_{e, \psi} \mid e \in E\right\}$ is linearly independent if and only if $|F| \leq d|V(F)|-d c(F)+\sum_{X \in C(F)} d_{\rho}\langle X\rangle$ for any $F \subseteq E$.

\section{Applications}

As applications, we shall address two problems from discrete geometry. In the first problem we discuss the symmetric redrawing problem of symmetrically embedded graphs, called symmetric frameworks. We shall extend Whiteley's parallel redrawing theorem to the symmetric setting. In the second problem we discuss the symmetry-forced rigidity of symmetric frameworks and extend Laman's theorem concerning the rigidity of graphs.

The section is organized as follows. We shall first introduce notions of symmetric graphs and symmetric frameworks in 6.1 and 6.2 respectively. Then, we will discuss the symmetric parallel redrawing problem in $\$ 6.3$ and the symmetry-forced rigidity in 6.4 .

6.1. Symmetric graphs. Let $H$ be a simple graph which may not be finite. An automorphism of $H$ is a permutation $\pi: V(H) \rightarrow V(H)$ such that $\{u, v\} \in E(H)$ if and only if $\{\pi(u), \pi(v)\} \in E(H)$. The set of all automorphisms of $H$ forms a subgroup of the symmetric group of $V(H)$, known as the automorphism group $\operatorname{Aut}(H)$ of $H$. An action of a group $\Gamma$ on $H$ is a group homomorphism $\theta: \Gamma \rightarrow$ $\operatorname{Aut}(H)$. An action $\theta$ is called free if $\theta(\gamma)(v) \neq v$ for any $v \in V$ and any nonidentity $\gamma \in \Gamma$. We say that a graph $H$ is $(\Gamma, \theta)$-symmetric (or simply $\Gamma$-symmetric) if $\Gamma$ acts on $H$ by $\theta$. In the subsequent discussion, we only consider free actions, and we omit specifying the action $\theta$ if it is clear from the context. We then denote $\theta(\gamma)(v)$ by $\gamma v$.

For a $\Gamma$-symmetric graph $H$, the quotient graph $H / \Gamma$ is a multigraph on the set $V(H) / \Gamma$ of vertex orbits, together with the set $E(H) / \Gamma$ of edge orbits as the edge set (with respect to $\theta$ ). An edge orbit may be represented by a loop in $H / \Gamma$. Figure 1 illustrates an example when $\Gamma$ is the dihedral group of order 8 .
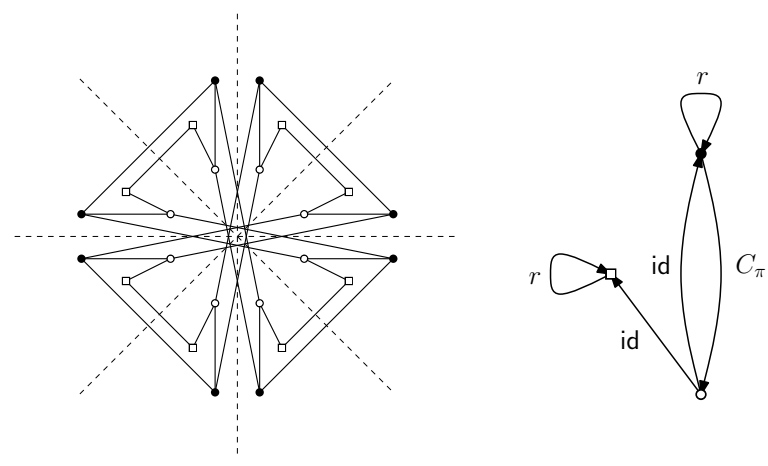

FiguRE 1. A symmetric graph and the quotient gain graph with dihedral group symmetry.

Several distinct graphs may have the same quotient graph. However, if we assume that the underlying action is free, then a gain labeling makes the relation one-to-one. To see this, we arbitrarily choose a vertex $v$ as a representative vertex from each 
vertex orbit. Then, each orbit is written by $\Gamma v=\{g v \mid g \in \Gamma\}$. If the action is free, an edge orbit connecting $\Gamma u$ and $\Gamma v$ in $H / \Gamma$ can be written by $\{\{g u, g h v\} \mid g \in \Gamma\}$ for a unique $h \in \Gamma$. We then orient the edge orbit from $\Gamma u$ to $\Gamma v$ in $H / \Gamma$ and assign to it gain $h$. In this way, we obtain the quotient $\Gamma$-gain graph, denoted by $(H / \Gamma, \psi)$.

Conversely, let $(G, \psi)$ be a finite $\Gamma$-gain graph for a group $\Gamma$. We simply denote the pair $(g, v)$ of $g \in \Gamma$ and $v \in V(G)$ by $g v$. The covering graph (also known as the derived graph) of $(G, \psi)$ is the simple graph with the vertex set $\Gamma \times V(G)=$ $\{g v \mid g \in \Gamma, v \in V(G)\}$ and the edge set $\{\{g u, g \psi(e) v\} \mid e=(u, v) \in E(G), g \in \Gamma\}$.

Clearly, $\Gamma$ freely acts on the covering graph with the action $\theta$ defined by $\theta(g)$ : $v \mapsto g v$ for $g \in \Gamma$, under which the quotient graph comes back to $(G, \psi)$. In this way, there is a one-to-one correspondence between $\Gamma$-symmetric graphs with free actions and $\Gamma$-gain graphs up to the equivalence of $\psi$. For more properties of covering graphs, see, e.g., [2,14.

6.2. Finite symmetric frameworks. A d-dimensional framework (or, simply, a framework) is a pair $(H, p)$ of a simple undirected graph $H$ and a mapping $p: V(H) \rightarrow \mathbb{R}^{d}$, called a point-configuration, which may be regarded as a straightline realization of $G$ in $\mathbb{R}^{d}$. In this paper, we are interested in symmetrically embedded symmetric graphs in the Euclidean space. Thus, throughout applications in $\sqrt{6}$, $\Gamma$ denotes a subgroup of matrix group $G L\left(\mathbb{R}^{d}\right)$.

Let $H$ be a $(\Gamma, \theta)$-symmetric graph, where $\Gamma$ freely acts on $H$ through $\theta$. A function $f: V(H) \rightarrow \mathbb{R}^{d}$ is called ( $\left.\Gamma, \theta\right)$-symmetric (or simply $\Gamma$-symmetric) if

$$
\gamma f(v)=f(\gamma v) \quad \forall \gamma \in \Gamma \text { and } \forall v \in V(H) .
$$

The pair $(H, p)$ is said to be a $(\Gamma, \theta)$-symmetric framework (or simply a $\Gamma$-symmetric framework) if $H$ and $p$ are $(\Gamma, \theta)$-symmetric.

It is convenient to fix a representative vertex $v$ of each vertex orbit $\Gamma v$ and define the quotient $f / \Gamma: V / \Gamma \rightarrow \mathbb{R}^{d}$ of $f$ to be $(f / \Gamma)(\Gamma v)=f(v)$ for each edge orbit $\Gamma v$ and its representative vertex $v$.

In the subsequent discussion, we shall only consider finite frameworks, where $H$ is a finite graph. (In $₫ 10$ we will discuss infinite frameworks with crystallographic symmetry.) Namely, we shall restrict our attention to discrete point groups $\mathcal{P}$, which are finite discrete subgroups of the orthogonal group $\mathcal{O}\left(\mathbb{R}^{d}\right)$, i.e., the set of $d \times d$ orthogonal matrices.

For a discrete point group $\Gamma$, let $\mathbb{Q}_{\Gamma}$ be the field generated by $\mathbb{Q}$ and the entries of matrices contained in $\Gamma$. For a $\Gamma$-gain graph $(G, \psi)$, a mapping $f: V(G) \rightarrow \mathbb{R}^{d}$ is said to be $\Gamma$-generic if the set of coordinates of the image of $f$ is algebraically independent over $\mathbb{Q}_{\Gamma}$. Also, for a $\Gamma$-symmetric graph $H$, a $\Gamma$-symmetric function $f: V(H) \rightarrow \mathbb{R}^{d}$ is said to be $\Gamma$-generic if $f / \Gamma$ is $\Gamma$-generic.

\subsection{Symmetric parallel redrawing problem.}

6.3.1. Parallel redrawing. Let $(H, p)$ be a finite $d$-dimensional framework. We shall consider $(H, p)$ as a drawing of graph $H$ in $\mathbb{R}^{d}$ with straight-line edges. A framework $(H, q)$ is called a parallel redrawing of $(H, p)$ if $q(i)-q(j)$ is parallel to $p(i)-p(j)$ for all $\{i, j\} \in E(H)$. No matter how dense the underlying graph is, any framework admits parallel redrawings, since a translation of $(H, p)$ or a dilation of $(H, p)$ is always a redrawing. A drawing $(H, p)$ is said to be robust if any redrawing of $(H, p)$ is a consequence of translations and dilation of $(H, p)$. In the parallel redrawing problem, we are asked whether $(H, p)$ is robust or not. 
In the context of rigidity theory, this concept is known as the direction-rigidity of $d$-dimensional bar-joint frameworks $(H, p)$, where we are interested in directionconstraint rather than conventional length-constraint (which we will discuss in the next subsection), or the mixture of length and direction constraints (see, e.g., [16. 37, 42,).

Let us take a look at the formal definition. We define a relocation of $(H, p)$ by $m: V(H) \rightarrow \mathbb{R}^{d}$ such that

$$
m(i)-m(j) \text { is parallel to } p(i)-p(j) \quad \forall\{i, j\} \in E(H)
$$

or, equivalently,

$$
\langle m(i)-m(j), \alpha\rangle=0 \quad \forall \alpha \in \mathbb{R}^{d} \text { such that }\langle p(i)-p(j), \alpha\rangle=0
$$

for all $\{i, j\} \in E(H)$, where $\langle\cdot, \cdot\rangle$ denotes the standard inner product in $\mathbb{R}^{d}$. For $t \in \mathbb{R}^{d}$, let us define a constant map $m_{t}: V(H) \rightarrow \mathbb{R}^{d}$ by $m_{t}(v)=t$ for $v \in V(H)$. Then, $m_{t}$ is a relocation of $(H, p)$, known as a translation. On the other hand, let $m_{\mathrm{di}}(v)=p(v)$ for $v \in V(H)$. Then, $m_{\mathrm{di}}$ is also a relocation of $(H, p)$, known as a dilation. In general, a relocation is called trivial if it is a linear combination of $m_{\mathrm{di}}$ and $m_{t}$ for $t \in \mathbb{R}^{d}$, and $(H, p)$ is called robust if all possible relocations are trivial. The set of trivial relocations of $(H, p)$ forms a linear subspace of $\left(\mathbb{R}^{d}\right)^{V}$, denoted by $\operatorname{tri}(H, p)$, which has dimension $d+1$ unless $\{p(v) \mid v \in V(H)\}$ is a point.

In [41, 42, Whiteley showed a combinatorial characterization of robust frameworks on generic point-configurations as a corollary of a combinatorial characterization of reconstructivity of pictures appearing in scene analysis. The goal of this section is to extend this result to the symmetric parallel redrawing problem of symmetric frameworks.

Let $\Gamma$ be a discrete point group in $\mathcal{O}\left(\mathbb{R}^{d}\right)$, and suppose that $(H, p)$ is $\Gamma$-symmetric. It is then natural to ask whether there is a redrawing preserving the symmetry. It is straightforward from (6.1), (6.2) to see the following:

Proposition 6.1. Let $(H, p)$ be a $\Gamma$-symmetric framework, and let $m: V \rightarrow \mathbb{R}^{d}$ be a $\Gamma$-symmetric relocation of $(H, p)$. Then, $(H, p+m)$ is $\Gamma$-symmetric and a parallel redrawing of $(H, p)$. Conversely, if $(H, q)$ is a $\Gamma$-symmetric parallel redrawing of $(H, p)$, then $q-p$ is a $\Gamma$-symmetric relocation of $(H, p)$.

We thus say that $(H, p)$ is symmetrically robust if any $\Gamma$-symmetric relocation of $(H, p)$ is trivial. The set of $\Gamma$-symmetric trivial relocations forms a linear subspace of $\operatorname{tri}(H, p)$, which is denoted by $\operatorname{tri}_{\Gamma}(H, p)$.

Recall that $\operatorname{dim}_{\mathbb{R}}(\operatorname{tri}(H, p))=d+1$. However, not every translation $m_{t}$ is $\Gamma$ symmetric; $m_{t}$ is $\Gamma$-symmetric if and only if $\gamma t=t$ for all $\gamma \in \Gamma$, or equivalently $t \in$ $\bigcap_{\gamma \in \Gamma} \operatorname{ker}\left(\gamma-I_{d}\right)$. Thus, if $\{p(v) \mid v \in V(H)\}$ is not the orbit of single point, $(H, p)$ is symmetrically robust if and only if the dimension of the space of $\Gamma$-symmetric relocations is equal to

$$
\operatorname{dim}_{\mathbb{R}} \operatorname{tri}_{\Gamma}(H, p)=1+\operatorname{dim}_{\mathbb{R}} \bigcap_{\gamma \in \Gamma} \operatorname{ker}\left(\gamma-I_{d}\right) .
$$

Note that the dilation is always $\Gamma$-symmetric, which is indeed crucial in the proof of our main claim below. Since $\Gamma$ is finite, $\operatorname{dim}_{\mathbb{R}} \bigcap_{\gamma \in \Gamma} \operatorname{ker}\left(\gamma-I_{d}\right)=\frac{1}{|\Gamma|} \sum_{\gamma \in \Gamma} \operatorname{trace}(\gamma)$.

6.3.2. Symmetric parallel redrawing polymatroids. For a symmetric relocation of $\Gamma$-symmetric framework $(H, p)$, the system (6.2) is apparently redundant due to 
the $\Gamma$-symmetry of $p$ and $m$, and the redundancy can be eliminated by using the quotient $\Gamma$-gain graph $(H / \Gamma, \psi)$.

To see this, let us take a representative vertex $v \in V(H)$ from each vertex orbit $\Gamma v \in V(H / \Gamma)$, as mentioned in $\$ 6.2$ Then, there is a natural one-to-one correspondence between $p$ and its quotient $p / \Gamma$ (resp., $m$ and $m / \Gamma$ ) through $p(v)=$ $p / \Gamma(\Gamma v)$ (resp., $m(v)=m / \Gamma(\Gamma v))$. Recall also that each edge orbit connecting from $\Gamma i$ to $\Gamma j$ is written by $\Gamma e=\left\{\left\{\gamma i, \gamma \psi_{e} j\right\}: \gamma \in \Gamma\right\}$, where $\psi_{e}$ is the gain of $\Gamma e$ in the quotient gain graph. Hence, 6 is.2) is written by

$$
\left\langle m(\gamma i)-m\left(\gamma \psi_{e} j\right), \alpha\right\rangle=0 \quad \forall \alpha \in \mathbb{R}^{d} \text { such that }\left\langle p(\gamma i)-p\left(\gamma \psi_{e} j\right), \alpha\right\rangle=0
$$

for each edge $\left\{\gamma i, \gamma \psi_{e} j\right\}$ in each edge orbit $\Gamma e$. Since $\Gamma$ consists of orthogonal matrices, the conditions over edges in $\Gamma e$ can be reduced to one condition,

$$
\left\langle m(i)-m\left(\psi_{e} j\right), \alpha\right\rangle=0 \quad \forall \alpha \in \mathbb{R}^{d} \text { such that }\left\langle p(i)-p\left(\psi_{e} j\right), \alpha\right\rangle=0 .
$$

By the $\Gamma$-symmetry of $p$ and $m$, this is further converted to

$\left\langle m / \Gamma(\Gamma i)-\psi_{e} m / \Gamma(\Gamma j), \alpha\right\rangle=0 \quad \forall \alpha \in \mathbb{R}^{d}$ such that $\left\langle p / \Gamma(\Gamma i)-\psi_{e} p / \Gamma(\Gamma j), \alpha\right\rangle=0$.

Therefore, to analyze the space of relocations $m$, it suffices to analyze the dimension of $m / \Gamma$ satisfying (6.4) for all edge orbits $\Gamma e=(\Gamma i, \Gamma j)$.

Thus, by simplifying notation, the problem can be considered in a general $\Gamma$-gain graph $(G, \psi)$, and our goal is to understand the space of $m \in\left(\mathbb{R}^{d}\right)^{V(G)}$ satisfying

$$
\left\langle m(i),-\psi_{e} m(j), \alpha\right\rangle=0 \quad \forall \alpha \in \mathbb{R}^{d} \text { such that }\left\langle p(i)-\psi_{e} p(j), \alpha\right\rangle=0
$$

for every $e=(i, j) \in E(G)$ with $\psi(e)=\psi_{e}$. To do that, we associate a $(d-1)$ dimensional linear subspace $P_{e, \psi}(p)$ with each edge orbit $e=(i, j) \in E(G)$ defined by

$$
P_{e, \psi}(p)=A_{e, \psi} \cap\left\{x \in\left(\mathbb{R}^{d}\right)^{V(G)} \mid\left\langle p(i)-\psi_{e} p(j), x(i)\right\rangle=0\right\}
$$

where $A_{e, \psi}$ is, as defined in (5.9), (5.10),

$$
A_{e, \psi}=\left\{\begin{array}{l|l}
x \in\left(\mathbb{R}^{d}\right)^{V(G)} & \begin{array}{l}
x(i)+\psi_{e} x(j)=0 \\
x(V \backslash\{i, j\})=0
\end{array}
\end{array}\right\}
$$

or

$$
A_{e, \psi}=\left\{x \in\left(\mathbb{R}^{d}\right)^{V(G)} \mid \exists \alpha \in \mathbb{R}^{d}: \begin{array}{l}
x(i)=\left(I_{d}-\psi_{e}\right) \alpha, \\
x(V \backslash\{i\})=0
\end{array}\right\}
$$

depending on whether $e$ is a nonloop or a loop, respectively. Observe then that $m \in\left(\mathbb{R}^{d}\right)^{V(G)}$ satisfies (6.5) for all $e \in E(G)$ if and only if $m$ is in the orthogonal complement of $\operatorname{span}\left\{P_{e, \psi}(p) \mid e \in E(G)\right\}$, because, for any $x \in P_{e, \psi}(p)$, we have

$$
\begin{aligned}
\langle m, x\rangle & =\langle m(i), x(i)\rangle+\langle m(j), x(j)\rangle \\
& =\langle m(i), x(i)\rangle-\left\langle m(j), \psi_{e}^{-1} x(i)\right\rangle \\
& =\left\langle m(i)-\psi_{e} m(j), x(i)\right\rangle
\end{aligned}
$$

with $\left\langle p(i)-\psi_{e} p(j), x(i)\right\rangle=0$. In total, we proved the following.

Theorem 6.2. Let $(H, p)$ be a $\Gamma$-symmetric framework and $(H / \Gamma, \psi)$ the quotient $\Gamma$-gain graph of $H$. Then, $(H, p)$ is symmetrically robust if and only if

$$
\operatorname{dim}_{\mathbb{R}}\left\{P_{e, \psi}(p / \Gamma) \mid e \in E(H / \Gamma)\right\}=d|V / \Gamma|-1-\frac{1}{|\Gamma|} \sum_{\gamma \in \Gamma} \operatorname{trace}(\gamma)
$$


6.3.3. Combinatorial characterization. By Theorem 6.2, it now suffices to analyze the polymatroid of $\Gamma$-gain graphs $(G, \psi)$ with linear representation $e \mapsto P_{e, \psi}(p)$ for $p: V(G) \rightarrow \mathbb{R}^{d}$, which we call the $\Gamma$-symmetric parallel redrawing polymatroid of $(G, \psi)$ (with respect to $p$ ). The following theorem provides a combinatorial characterization of this polymatroid.

Theorem 6.3. Let $\Gamma$ be a discrete point group with the identity map $\rho: \gamma \in \Gamma \mapsto$ $\gamma \in G L\left(\mathbb{R}^{d}\right),(G=(V, E), \psi)$ a $\Gamma$-gain graph, and $p: V \rightarrow \mathbb{R}^{d}$ a $\Gamma$-generic mapping. Define $f_{\rho}$ as in (15.12) by

$$
f_{\rho}(F)=d|V(F)|-d c(F)+\sum_{X \in C(F)} \operatorname{dim}_{\mathbb{R}}\left\{\operatorname{image}\left(I_{d}-\rho(\gamma)\right) \mid \gamma \in X\right\} \quad(F \subseteq E)
$$

and $h_{\rho}: 2^{E} \rightarrow \mathbb{Z}$ by

$$
h_{\rho}(F)=f_{\rho}(F)-1 \quad(F \subseteq E) .
$$

Then,

$$
\operatorname{dim}_{\mathbb{R}}\left\{P_{e, \psi}(p) \mid e \in E\right\}=\hat{h}_{\rho}(E) .
$$

In other words, for almost all $p$, the $\Gamma$-symmetric parallel redrawing polymatroid is equal to the polymatroid induced by $h_{\rho}$.

Proof. The proof idea is from the alternative proof of Laman's theorem by Lovász and Yemini 20].

Applying Theorem 5.3 with $f_{\rho}$, the polymatroid $\mathbf{P}\left(f_{\rho}\right)=\left(E, f_{\rho}\right)$ is equal to the linear polymatroid $\mathbf{D P}_{\rho}(G, \psi)$ with the linear representation $e \mapsto A_{e, \psi}$ (given in (6.7), (6.8) ).

We define a hyperplane $\mathcal{H}$ of $\left(\mathbb{R}^{d}\right)^{V}$ (i.e., a $(d|V|-1)$-dimensional subspace) by

$$
\mathcal{H}=\left\{x \in\left(\mathbb{R}^{d}\right)^{V} \mid\langle p, x\rangle=0\right\} .
$$

Then, observe that $P_{e, \psi}(p)=A_{e, \psi} \cap \mathcal{H}$ for every $e \in E$. Indeed, for any $e=(i, j) \in$ $E$ and any $x \in A_{e, \psi}$, we have $\langle p, x\rangle=\langle p(i), x(i)\rangle+\langle p(j), x(j)\rangle=\langle p(i), x(i)\rangle+$ $\left\langle p(j),-\psi_{e}^{-1} x(i)\right\rangle=\left\langle p(i)-\psi_{e} p(j), x(i)\right\rangle$, which implies that $x \in \mathcal{H}$ if and only if $\left\langle p(i)-\psi_{e} p(j), x(i)\right\rangle=0$. Therefore, as $p$ is $\Gamma$-generic, we conclude that the $\Gamma$ symmetric parallel redrawing polymatroid of $(G, \psi)$ is obtained from $\mathbf{D P}_{\rho}(G, \psi)$ by a Dilworth truncation, given in $\$ 3.3 .2$, By Theorem $[3.2$ and Theorem $[5.3$, we obtain

$$
\begin{aligned}
& \operatorname{dim}_{\mathbb{R}}\left\{P_{e, \psi}(p) \mid e \in E\right\} \\
& =\min \left\{\sum_{i}\left(\operatorname{dim}_{\mathbb{R}}\left\{A_{e, \psi} \mid e \in E_{i}\right\}-1\right) \mid \operatorname{arpartition}\left\{E_{1}, \ldots, E_{k}\right\} \text { of } E\right\} \\
& \text { (by Theorem 3.2) } \\
& =\min \left\{\sum_{i}\left(f_{\rho}\left(E_{i}\right)-1\right) \mid \text { a partition }\left\{E_{1}, \ldots, E_{k}\right\} \text { of } E\right\} \quad \text { (by Theorem } 5.3 \text { ) } \\
& \left.=\min \left\{\sum_{i} h_{\rho}\left(E_{i}\right) \mid \text { a partition }\left\{E_{1}, \ldots, E_{k}\right\} \text { of } E\right\} \quad \text { (by (6.9) }\right) \\
& =\hat{h}_{\rho}(E) . \quad(\text { by }(3.1)) \text {. }
\end{aligned}
$$

The following extends the result of Whiteley [42] to the symmetric parallel redrawing problem, which directly follows from Theorem 6.2 and Theorem 6.3. 
Corollary 6.4. Let $H$ be a $\Gamma$-symmetric graph for a discrete point group $\Gamma$ and $(H / \Gamma, \psi)$ the quotient $\Gamma$-gain graph. For almost all $\Gamma$-symmetric $p: V(H) \rightarrow$ $\mathbb{R}^{d},(H, p)$ is symmetrically robust if and only if the graph obtained from $H / \Gamma$ by replacing each edge $e \in E(H / \Gamma)$ by $d-1$ parallel copies contains an edge subset $I$ satisfying the following counting conditions:

- $|I|=d|V|-1-\frac{1}{|\Gamma|} \sum_{\gamma \in \Gamma} \operatorname{trace}(\gamma)$;

- $|F| \leq d|V(F)|-d c(F)-1+\sum_{X \in C(F)} \operatorname{dim}_{\mathbb{R}}\left\{\operatorname{image}\left(I_{d}-\gamma\right) \mid \gamma \in\langle X\rangle\right\}$ for any nonempty $F \subseteq I$.

6.4. Symmetry-forced rigidity of symmetric frameworks. We then move to another application of Theorem [5.3. the infinitesimal rigidity of symmetric frameworks. The papers by Schulze and Whiteley [36] and Jordán et al. [17] contain a more detailed explanation on this topic.

6.4.1. Symmetry-forced infinitesimal rigidity. The infinitesimal rigidity concerns the dimension of the space of infinitesimal motions. An infinitesimal motion of a framework $(H, p)$ is defined as an assignment $m: V(H) \rightarrow \mathbb{R}^{d}$ such that

$$
\langle m(i)-m(j), p(i)-p(j)\rangle=0 \quad \forall\{i, j\} \in E(H) .
$$

The set of infinitesimal motions forms a linear space, denoted $L(H, p)$.

In general, for a set $P \subseteq \mathbb{R}^{d}$ of points, an infinitesimal isometry of $P$ is defined by $m: P \rightarrow \mathbb{R}^{d}$ such that

$$
\langle m(x)-m(y), x-y\rangle=0 \quad \forall x, y \in P .
$$

The set of infinitesimal isometries forms a linear space, denoted by iso $(P)$. Notice that for a skew-symmetric matrix $S$ and $t \in \mathbb{R}^{d}$, a mapping $m: P \rightarrow \mathbb{R}^{d}$ defined by

$$
m(x)=S x+t \quad(x \in P)
$$

is an infinitesimal isometry of $P$. Indeed, it is well-known that any infinitesimal isometry can be described in this form and

$$
\operatorname{dim}_{\mathbb{R}} \operatorname{iso}(P)=d(k+1)-\left(\begin{array}{c}
k+1 \\
2
\end{array}\right),
$$

where $k$ denotes the affine dimension of $P$. For example, for $d=2$, an infinitesimal isometry is a linear combination of translations and the infinitesimal rotation around the origin.

An infinitesimal motion $m: V(H) \rightarrow \mathbb{R}^{d}$ of a framework $(H, p)$ is said to be trivial if $m$ can be expressed by

$$
m(v)=S p(v)+t \quad(v \in V(H))
$$

for some skew-symmetric matrix $S$ and $t \in \mathbb{R}^{d}$. The set of all trivial motions forms a linear subspace of $L(H, p)$, denoted by $\operatorname{tri}(H, p)$. By definition, $\operatorname{tri}(H, p)$ is isomorphic to iso $(\{p(v) \mid v \in V(H)\})$, and hence (6.11) gives the exact dimension of $\operatorname{tri}(H, p) .(H, p)$ is called infinitesimally rigid if $L(H, p)=\operatorname{tri}(H, p)$.

As in the parallel redrawing problem, we are interested in $\Gamma$-symmetric infinitesimal motions of symmetric frameworks. For a discrete point group $\Gamma$, a $\Gamma$-symmetric framework $(H, p)$ is said to be symmetry-forced rigid if any $\Gamma$-symmetric infinitesimal motion of $(H, p)$ is trivial. We should remark that, as in the case of the parallel redrawing problem, not every trivial infinitesimal motion is $\Gamma$-symmetric. 
The following result of Schulze 34 motivates us to look at symmetry-forced infinitesimal rigidity. (The precise definition of some terminologies are omitted here.)

Theorem 6.5 (Schulze 34]). Let $\Gamma$ be a discrete point group and $H$ a $\Gamma$-symmetric graph. Then, for any $\Gamma$-generic $p,(H, p)$ has a nontrivial continuous motion that preserves the $\Gamma$-symmetry if and only if $(H, p)$ has a nontrivial $\Gamma$-symmetric infinitesimal motion.

6.4.2. Orbit rigidity matrix. Let $(H, p)$ be a $\Gamma$-symmetric framework. Due to $\Gamma$ symmetry, the system (6.10) of linear equations (with respect to $m$ ) is redundant. Schulze and Whiteley [36] pointed out that the system can be reduced to $|E(H) / \Gamma|$ linear equations.

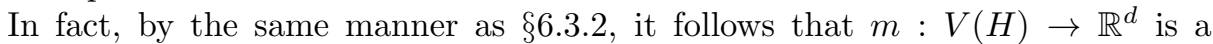
$\Gamma$-symmetric infinitesimal motion of $(H, p)$ if and only if

$$
\left\langle m / \Gamma(\Gamma i), p / \Gamma(\Gamma i)-\psi_{e} \cdot p / \Gamma(\Gamma j)\right\rangle+\left\langle m / \Gamma(\Gamma j), p / \Gamma(\Gamma j)-\psi_{e}^{-1} \cdot p / \Gamma(\Gamma i)\right\rangle=0
$$

for every oriented edge orbit $\Gamma e=(\Gamma i, \Gamma j)$ in the quotient gain graph $(H / \Gamma, \psi)$. By regarding (6.13) as a system of linear equations of $m / \Gamma$, the corresponding $|E(H) / \Gamma| \times d|V(H) / \Gamma|$-matrix is called the orbit rigidity matrix by Schulze and Whiteley 36.

In general, for a $\Gamma$-gain graph $(G, \psi)$ and $p: V(G) \rightarrow \mathbb{R}^{d}$, we are interested in the system of linear equations on $m \in\left(\mathbb{R}^{d}\right)^{V(G)}$ defined by

$$
\left\langle m(i), p(i)-\psi_{e} p(j)\right\rangle+\left\langle m(j), p(j)-\psi_{e}^{-1} p(i)\right\rangle=0 \quad \forall e=(i, j) \in E(G) .
$$

To analyze the solution space of (6.14), we then associate a 1-dimensional linear space $R_{e, \psi}$ with each $e=(i, j) \in E(G)$,

$$
R_{e, \psi}(p)=\left\{x \in\left(\mathbb{R}^{d}\right)^{V(G)} \mid \exists t \in \mathbb{R}: \begin{array}{l}
x(i)=t\left(p(i)-\psi_{e} p(j)\right), \\
x(V)=t\left(p(j)-\psi_{e}^{-1} p(i)\right), \\
x(i, j\})=0
\end{array}\right\}
$$

if $e$ is a nonloop edge, and

$$
R_{e, \psi}(p)=\left\{x \in\left(\mathbb{R}^{d}\right)^{V(G)} \mid \exists t \in \mathbb{R}: \begin{array}{l}
x(i)=t\left(2 I_{d}-\psi_{e}-\psi_{e}^{-1}\right) p(i), \\
x(V \backslash\{i\})=0
\end{array}\right\}
$$

if $e$ is a loop attached to $i$. Observe then that $m$ satisfies (6.14) if and only if $m$ is in the orthogonal complement of $\operatorname{span}\left\{R_{e, \psi}(p) \mid e \in E(G)\right\}$. This implies the following.

Proposition 6.6 (Schulze and Whiteley [36]). Let $(H, p)$ be a $\Gamma$-symmetric framework and $(H / \Gamma, \psi)$ be the quotient $\Gamma$-gain graph. Then, the dimension of the space of $\Gamma$-symmetric infinitesimal motions of $(H, p)$ is equal to

$$
d|V(H) / \Gamma|-\operatorname{dim}_{\mathbb{R}}\left\{R_{e, \psi}(p / \Gamma) \mid e \in E(H / \Gamma)\right\} .
$$

The detailed description and examples can be found in [34,36]. A combinatorial necessity condition for symmetry-forced rigidity can be found in [17.

6.4.3. Combinatorial characterization. The following theorem provides a combinatorial characterization of the linear matroid induced on $\left\{R_{e, \psi}(p) \mid e \in E(G)\right\}$ for the spacial case of $d=2$ and rotation groups $\mathcal{C}_{k}$. 
Theorem 6.7. Let $\mathcal{C}_{k}$ be the group of $k$-fold rotations around the origin in the plane. Let $(G=(V, E), \psi)$ be a $\mathcal{C}_{k}$-gain graph and $p: V \rightarrow \mathbb{R}^{2}$ be a $\mathcal{C}_{k}$-generic mapping. Then,

$$
\operatorname{dim}_{\mathbb{R}}\left\{R_{e, \psi}(p) \mid e \in E\right\}=\hat{h}_{\rho}(E),
$$

where $h_{\rho}$ is as defined in (6.9) with $\Gamma=\mathcal{C}_{k}$.

Proof. The proof technique is exactly the same as the proof of Theorem 6.3.

Let $C_{\pi / 2}$ be the matrix of size $2 \times 2$, representing the 4 -fold rotation around the origin in the Euclidean plane. We define a hyperplane $\mathcal{H}^{\prime}$ of $\left(\mathbb{R}^{2}\right)^{V}$ by

$$
\mathcal{H}^{\prime}=\left\{x \in\left(\mathbb{R}^{2}\right)^{V} \mid \sum_{v \in V}\left\langle C_{\pi / 2} p(v), x(v)\right\rangle=0\right\}
$$

where $p \in\left(\mathbb{R}^{2}\right)^{V}$ is $\mathcal{C}_{k}$-generic as defined in the statement.

Let $A_{e, \psi}$ be the 2-dimensional linear subspace defined in (6.7), (6.8) with $d=2$. Then, observe that for each nonloop $e=(i, j) \in E$ and for any $x \in A_{e, \psi}$,

$$
\begin{aligned}
\sum_{v \in V}\left\langle C_{\pi / 2} p(v), x(v)\right\rangle & =\left\langle C_{\pi / 2} p(i), x(i)\right\rangle+\left\langle C_{\pi / 2} p(j), x(j)\right\rangle \\
& =\left\langle C_{\pi / 2} p(i), x(i)\right\rangle+\left\langle C_{\pi / 2} p(j),-\psi_{e}^{-1} x(i)\right\rangle \\
& =\left\langle C_{\pi / 2}\left(p(i)-\psi_{e} p(j)\right), x(i)\right\rangle
\end{aligned}
$$

where we used the fact that $C_{\pi / 2}$ commutes with any element of $\mathcal{C}_{k}$. This implies that $x \in \mathcal{H}^{\prime}$ if and only if $x(i) \in \operatorname{span}\left\{p(i)-\psi_{e} p(j)\right\}$ for every $e=(i, j) \in E$. In other words, $R_{e, \psi}(p)=A_{e, \psi} \cap \mathcal{H}^{\prime}$. The same analysis works in case of loops $e$.

Since $p$ is $\mathcal{C}_{k}$-generic, we conclude that the linear matroid induced on $\left\{R_{e, \psi}(p) \mid\right.$ $e \in E\}$ is obtained from $\mathbf{D P}_{\rho}(G, \psi)$ by a Dilworth truncation. Since $\mathbf{D P}_{\rho}(G, \psi)=$ $\mathbf{P}\left(f_{\rho}\right)$ by Theorem 5.3 , Theorem 3.2 implies the statement.

Combining Proposition 6.6 and Theorem 6.7, we conclude that the row matroid of an orbit rigidity matrix is the matroid induced by $h_{\rho}$ if $d=2$ and the underlying symmetry is a group of rotations. The same characterization was obtained in [17,21] by different approaches.

\section{Matroids induCED By GROUP ACTIONS ON EXTERIOR POWERS}

In this section, we give another application to rigidity theory, where we extend Tay's theorem 39 on generic rigidity of body-bar frameworks to a symmetric setting. The result solves a conjecture given in [29. For this extension, we shall first investigate the case when a group is represented in the exterior power of a vector space.

7.1. Restriction to decomposable $k$-vectors. In the subsequent discussion of this section, the underlying group $\Gamma$ is equipped with a linear representation $\rho$ : $\Gamma \rightarrow G L\left(\mathbb{F}^{d}\right)$ over a filed $\mathbb{F}$. As before, we denote by $\mathbb{K}$ a field obtained from $\mathbb{F}$ by transcendental extensions.

Let $\bigwedge^{k} \mathbb{F}^{d}$ be the $k$-th exterior power of $\mathbb{F}^{d}$. Recall that $\bigwedge^{k} \mathbb{F}^{d}$ is a $\left(\begin{array}{l}d \\ k\end{array}\right)$-dimensional linear space, and so each entry of an element of $\bigwedge^{k} \mathbb{F}^{d}$ can be naturally indexed by a $k$-tuple $\left(i_{1}, \ldots, i_{k}\right)$ with $1 \leq i_{1}<\cdots<i_{k} \leq d$. An element of $\bigwedge^{k} \mathbb{F}^{d}$ is called a $k$-vector, and a $k$-vector is said to be decomposable if it can be written in the form $v_{1} \wedge \cdots \wedge v_{k}$ for some $v_{1}, \ldots, v_{k} \in \mathbb{F}^{d}$. 
Let us consider a natural action of $\Gamma$ on $\bigwedge^{k} \mathbb{F}^{d}$; that is, $\gamma \in \Gamma$ acts on a decomposable element $v_{1} \wedge \cdots \wedge v_{k}$ by $\rho(\gamma) v_{1} \wedge \cdots \wedge \rho(\gamma) v_{k}$ and extends linearly to the other elements. This leads to a linear mapping from $\bigwedge^{k} \mathbb{F}^{d}$ to $\bigwedge^{k} \mathbb{F}^{d}$. It is known that this action is a well-defined group representation of $\Gamma$ over $G L\left(\bigwedge^{k} \mathbb{F}^{d}\right)$. In other words, there is a unique representation $\rho^{(k)}: \Gamma \rightarrow G L\left(\bigwedge^{k} \mathbb{F}^{d}\right)$ such that $\rho^{(k)}(\gamma)\left(v_{1} \wedge \cdots \wedge v_{k}\right)=\rho(\gamma) v_{1} \wedge \cdots \wedge \rho(\gamma) v_{k}$ for each $\gamma \in \Gamma$ and each $v_{1} \wedge \cdots \wedge v_{k}$ (see, e.g., 15, Chapter 7]).

Note that $\rho^{(k)}(\gamma)$ is a matrix of size $\left(\begin{array}{l}d \\ k\end{array}\right) \times\left(\begin{array}{l}d \\ k\end{array}\right)$. To see a specific expression of the entries, let us simply denote $N=\rho(\gamma)$. For $1 \leq i_{1}<\cdots<i_{k} \leq d$ and $1 \leq j_{1}<\cdots<j_{k} \leq d$, let $N_{i_{1} \ldots i_{k}}^{j_{1} \ldots j_{k}}$ be the submatrix of $N$ induced by the $i_{1}$-th, $\ldots, i_{k}$-th rows and the $j_{1}$-th $\ldots, j_{k}$-th columns. If we index each column and each row of $N^{(k)}$ by a $k$-tuple $\left(i_{1}, \ldots, i_{k}\right)$ according to the index ordering of elements in $\bigwedge^{k} \mathbb{F}^{d}$, we have

$$
N^{(k)}\left[\left(j_{1}, \ldots, j_{k}\right),\left(i_{1}, \ldots, i_{k}\right)\right]=\operatorname{det} N_{j_{1} \ldots j_{k}}^{i_{1} \ldots i_{k}},
$$

where $N^{(k)}\left[\left(j_{1}, \ldots, j_{k}\right),\left(i_{1}, \ldots, i_{k}\right)\right]$ denotes the entry at the $\left(j_{1}, \ldots, j_{k}\right)$-th row and $\left(i_{1}, \ldots, i_{k}\right)$-th column.

Using representation $\rho^{(k)}$, we now consider a special case of matroids given in 5.2. Let $(G=(V, E), \psi)$ be a $\Gamma$-gain graph with a gain function $\psi: e \mapsto \psi_{e}$. For each $e=(i, j) \in E$, let us assign $x_{e, \psi}^{(k)} \in\left(\bigwedge^{k} \mathbb{K}^{d}\right)^{V}$ as follows:

$$
x_{e, \psi}^{(k)}(v)= \begin{cases}-\rho^{(k)}\left(\psi_{e}\right) \alpha_{e} & \text { if } v=i \\ \alpha_{e} & \text { if } v=j \\ 0 & \text { otherwise }\end{cases}
$$

$$
x_{e, \psi}^{(k)}(v)= \begin{cases}\left(\rho^{(k)}\left(\psi_{e}\right)-I_{\left(\begin{array}{l}
d \\
k
\end{array}\right)}\right) \alpha_{e} & \text { if } v=i \\
0 & \text { otherwise }\end{cases}
$$

depending on whether $e$ is a nonloop edge or a loop, where $\alpha_{e}=\left(\alpha_{e}^{1}, \ldots, \alpha_{e}^{\left(\begin{array}{c}d \\ k\end{array}\right)}\right)^{\top} \in$ $\bigwedge^{k} \mathbb{K}^{d}$ such that $\left\{\alpha_{e}^{i} \mid 1 \leq i \leq\left(\begin{array}{l}d \\ k\end{array}\right), e \in E\right\}$ is algebraically independent over $\mathbb{F}$. Then, by Corollary 5.5, $\left\{x_{e, \psi}^{(k)} \mid e \in E\right\}$ is linearly independent if and only if $|F| \leq f_{\rho^{(k)}}(F)$ for any $F \subseteq E$ where

$$
\begin{aligned}
& f_{\rho^{(k)}}(F)=\left(\begin{array}{l}
d \\
k
\end{array}\right)|V(F)|-\left(\begin{array}{l}
d \\
k
\end{array}\right) c(F)+\sum_{X \in C(F)} d_{\rho^{(k)}}\langle X\rangle \quad(F \subseteq E), \\
& d_{\rho^{(k)}}(X)=\operatorname{dim}_{\mathbb{R}}\left\{\operatorname{image}\left(\rho^{(k)}(\gamma)-I_{\left(\begin{array}{c}
d \\
k
\end{array}\right)}\right) \mid \gamma \in X\right\} \quad(X \subseteq \Gamma) .
\end{aligned}
$$

Note that, for any $\gamma \in \Gamma$, we have $\operatorname{image}\left(\rho^{(k)}(\gamma)-I_{\left(\begin{array}{c}d \\ k\end{array}\right)}\right)=\operatorname{span}\left\{\rho(\gamma) p_{1} \wedge \cdots \wedge \rho(\gamma) p_{k}-p_{1} \wedge \cdots \wedge p_{k} \mid p_{1}, \ldots, p_{k} \in \mathbb{F}^{d}\right\}$, and hence $d_{\rho^{(k)}}$ can be rewritten in terms of decomposable $k$-vectors by putting this into (7.4). It is hence natural to ask whether the matroid $\mathbf{M}\left(f_{\rho^{(k)}}\right)$ also has a linear representation in terms of decomposable $k$-vectors, that is, a representation 
given by

$$
\hat{x}_{e, \psi}^{(k)}(v)= \begin{cases}-\rho\left(\psi_{e}\right) p_{e, 1} \wedge \cdots \wedge \rho\left(\psi_{e}\right) p_{e, k} & \text { if } v=i \\ p_{e, 1} \wedge \cdots \wedge p_{e, k} & \text { if } v=j \\ 0 & \text { otherwise }\end{cases}
$$

and

$$
\hat{x}_{e, \psi}^{(k)}(v)= \begin{cases}\rho\left(\psi_{e}\right) p_{e, 1} \wedge \cdots \wedge \rho\left(\psi_{e}\right) p_{e, k}-p_{e, 1} \wedge \cdots \wedge p_{e, k} & \text { if } v=i, \\ 0 & \text { otherwise }\end{cases}
$$

for some $p_{e, 1}, \ldots, p_{e, k} \in \mathbb{K}^{d}$. The next theorem asserts that (7.5), (17.6) indeed define a linear representation of $\mathbf{M}\left(f_{\rho^{(k)}}\right)$.

Theorem 7.1. For some $\left\{p_{e, i} \in \mathbb{K}^{d} \mid e \in E, 1 \leq i \leq k\right\}$, the linear matroid induced on $\left\{\hat{x}_{e, \psi}^{(k)} \mid e \in E\right\}$ is equal to $\mathbf{M}\left(f_{\rho^{(k)}}\right)$.

Proof. Let

$$
A_{e, \psi}^{(k)}=\left\{\begin{array}{l|l}
x \in\left(\bigwedge^{k} \mathbb{K}^{d}\right)^{V} \mid \begin{array}{l}
x(i)+\rho^{(k)}\left(\psi_{e}\right) x(j)=0, \\
x(V \backslash\{i, j\})=0
\end{array}
\end{array}\right\}
$$

for a nonloop edge $e \in E$ and

$$
A_{e, \psi}^{(k)}=\left\{x \in\left(\bigwedge^{k} \mathbb{K}^{d}\right)^{V} \mid \exists \alpha \in\left(\bigwedge^{k} \mathbb{K}^{d}\right)^{V}: \begin{array}{l}
x(i)=\left(I_{d}-\rho^{(k)}\left(\psi_{e}\right)\right) \alpha, \\
x(V \backslash\{i, j\})=0
\end{array}\right\}
$$

for a loop $e \in E$.

Let $\operatorname{Gr}(d, k)$ be the set of all decomposable $k$-vectors in $\bigwedge^{k} \mathbb{K}^{d} \cdot \operatorname{Gr}(d, k)$ is known as the Grassmannian in the literature and is an irreducible rational variety spanning $\bigwedge^{k} \mathbb{K}^{d}$. We shall define a subset $\hat{A}_{e, \psi}$ of $A_{e, \psi}$ by

$$
\hat{A}_{e, \psi}^{(k)}=A_{e, \psi}^{(k)} \cap\left\{x \in\left(\bigwedge^{k} \mathbb{K}^{d}\right)^{V} \mid x(j) \in \operatorname{Gr}(d, k)\right\}
$$

for a nonloop edge $e=(i, j)$ and

$$
\hat{A}_{e, \psi}^{(k)}=\left\{x \in\left(\bigwedge^{k} \mathbb{K}^{d}\right)^{V} \mid \exists \alpha \in \operatorname{Gr}(d, k): \begin{array}{l}
x(i)=\left(I_{d}-\rho^{(k)}\left(\psi_{e}\right)\right) \alpha \\
x(V \backslash\{i, j\})=0
\end{array}\right\}
$$

for a loop e. By Theorem [5.3. we know that $f_{\rho^{(k)}}(E)=\operatorname{dim}_{\mathbb{R}}\left\{A_{e, \psi}^{(k)} \mid e \in E\right\}$, and each linear representation of $\mathbf{M}\left(f_{\rho^{(k)}}\right)$ is obtained by taking a representative vector $x_{e, \psi}^{(k)}$ from each $A_{e, \psi}^{(k)}$ in generic position. Thus, to show the statement, it suffices to show that a representative vector $x_{e, \psi}^{(k)}$ can be taken from $\hat{A}_{e, \psi}^{(k)}$ so that $\left\{x_{e, \psi}^{(k)} \mid e \in E\right\}$ is in generic position in the sense of (3.5).

Suppose that $E$ has no loop. Then, each $\hat{A}_{e, \psi}^{(k)}$ is (linearly) isomorphic to $\operatorname{Gr}(d, k)$ by a projection to $x(j)$. Notice that the condition (3.5) of genericity is written in terms of linear dependencies. Since $\operatorname{Gr}(k, d)$ is an irreducible rational variety, the linear isomorphism between $\hat{A}_{e, \psi}^{(k)}$ and $\operatorname{Gr}(d, k)$ implies that a representative vertex can be taken from $\hat{A}_{e, \psi}^{(k)}$ in generic position. (A more detailed description for a special case can be found in [38, Theorem 3.1], and exactly the same argument can be applied here.)

If $e$ is a loop, then $A_{e, \psi}^{(k)}$ and $\hat{A}_{e, \psi}^{(k)}$ are linearly isomorphic to $\left(\rho^{(k)}\left(\psi_{e}\right)-I_{d}\right)\left(\bigwedge^{k} \mathbb{K}^{d}\right)$ and $\left(\rho^{(k)}\left(\psi_{e}\right)-I_{d}\right) \operatorname{Gr}(d, k)$, respectively. Since $\rho^{(k)}\left(\psi_{e}\right)-I_{d}$ is a linear operator, we can apply the same argument. 
7.2. Symmetry-forced rigidity of body-bar frameworks. As an application, we consider matroids arose in the rigidity of symmetric body-bar frameworks, which are structures consisting of rigid bodies linked by bars in $\mathbb{R}^{d}$.

Let $\operatorname{Aff}\left(\mathbb{R}^{d}\right)$ be the group of invertible affine transformations. It is well-known that $\operatorname{Aff}\left(\mathbb{R}^{d}\right)=G L\left(\mathbb{R}^{d}\right) \ltimes \mathbb{R}^{d}$, that is, the semidirect product of $G L\left(\mathbb{R}^{d}\right)$ and $\mathbb{R}^{d}$, and each element $\gamma=\left(A_{\gamma}, t_{\gamma}\right) \in \operatorname{Aff}\left(\mathbb{R}^{d}\right)$ acts on $\mathbb{R}^{d}$ by $\gamma \cdot q=A_{\gamma} q+t_{\gamma}$ for $q \in \mathbb{R}^{d}$.

The $d$-dimensional Euclidean group $\mathcal{E}(d)$ is a subgroup of $\operatorname{Aff}\left(\mathbb{R}^{d}\right)$, where $\left(A_{\gamma}, t_{\gamma}\right)$ $\in \operatorname{Aff}\left(\mathbb{R}^{d}\right)$ is in $\mathcal{E}(d)$ if and only if $A_{\gamma} \in \mathcal{O}\left(\mathbb{R}^{d}\right)$. A space group (or crystallographic group) $\Gamma$ is a discrete cocompact subgroup of $\mathcal{E}(d)$; i.e., $\mathbb{R}^{d} / \Gamma$ is compact. Throughout this subsection, $\Gamma$ denotes either a space group or a discrete point group, where $t_{\gamma}=0$ in case of a point group.

We now briefly take a look at how the linear matroid given in the last subsection arises in the context of rigidity of body-bar frameworks. The following modeling is based on [5]. A body-bar framework is a structure consisting of rigid bodies connected by bars, and it can be represented by a triple $(H, B, q)$, where

- $H$ is an undirected graph whose vertex corresponds to a body and whose edge corresponds to a bar liking the corresponding two bodies;

- $B$ indicates the location of each body corresponding to each vertex $v \in V$ by $B(v)=\left(A_{v}, p_{v}\right) \in \mathcal{O}\left(\mathbb{R}^{d}\right) \ltimes \mathbb{R}^{d}$ (i.e., each body is identified with a Cartesian (local) coordinate system);

- $q$ indicates the location of each bar in each local coordinate system as follows: for each $e \in E(H)$ and an endvertex $v$ of $e, q(e, v) \in \mathbb{R}^{d}$ denotes the coordinate of the endpoint of the bar corresponding to $e$ in the coordinate system of the body $v$. Thus, the coordinate in the global system is equal to $A_{v} q(e, v)+p_{v}$, denoted by $\tilde{q}(e, v)$.

$B$ and $q$ are called a body-configuration and a bar-configuration, respectively.

When bodies are moving, each bar constrains the distance between the endpoints. Such a length constraint can be written by

$$
\langle\tilde{q}(e, i)-\tilde{q}(e, j), \tilde{q}(e, i)-\tilde{q}(e, j)\rangle=\ell_{e} \quad \forall e=\{i, j\} \in E(H)
$$

where $\ell_{e}$ denotes the bar-length associated with $e$.

We consider a symmetric version of body-bar frameworks, where a body-bar framework $(H, B, q)$ is $\Gamma$-symmetric if $H$ is a $\Gamma$-symmetric graph with an action $\theta$ freely acting on $E(H)$ and $B$ and $q$ are subject to $\Gamma$-symmetry. For any $v \in V(H)$, $e \in E(H)$, and $\gamma=\left(A_{\gamma}, t_{\gamma}\right) \in \Gamma$,

$$
\begin{aligned}
B(\gamma v) & =\gamma B(v)=\left(A_{\gamma} A_{v}, A_{\gamma} p_{v}+t_{\gamma}\right), \\
q(\gamma e, \gamma v) & =q(e, v) .
\end{aligned}
$$

Indeed, in the global coordinate system, we have $\tilde{q}(\gamma e, \gamma v)=A_{\gamma} A_{v} q(\gamma e, \gamma v)+$ $A_{\gamma} p_{v}+t=A_{\gamma} A_{v} q(e, v)+A_{\gamma} p_{v}+t=\gamma \tilde{q}(e, v)$, and thus the definition implies the $\Gamma$-symmetry of $\tilde{q}$ in the global system.

By using $\Gamma$-symmetry of $q$ and $B$, the system (7.7) can be reduced to the following system of equations:

$$
\left\langle\tilde{q}(e, i)-\psi_{e} \tilde{q}(e, j), \tilde{q}(e, i)-\psi_{e} \tilde{q}(e, j)\right\rangle=\ell_{e} \quad \forall \Gamma e=(\Gamma i, \Gamma j) \in E(H / \Gamma),
$$

where $\psi(\Gamma e)=\psi_{e}$ denotes the gain of $\Gamma e$ in the quotient $\Gamma$-gain graph.

Thus, the analysis can be accomplished on the quotient graph, and we may consider the problem even in a general $\Gamma$-gain graph $(G=(V, E), \psi)$. Namely, 
given a $\Gamma$-gain graph $(G, \psi), B(v)=\left(A_{v}, p_{v}\right)$ for $v \in V$, and $q_{e, i}, q_{e, j} \in \mathbb{R}^{d}$ for $e=(i, j) \in E$, we consider the system

$$
\left\langle\tilde{q}_{e, i}-\psi_{e} \cdot \tilde{q}_{e, j}, \tilde{q}_{e, i}-\psi_{e} \cdot \tilde{q}_{e, j}\right\rangle=\ell_{e} \quad \forall e=(i, j) \in E .
$$

By taking the derivative with respect to $B$, we shall again investigate the infinitesimal rigidity. To see this, let us focus on the equation for $e=(i, j) \in E$ and simply denote $q_{e, i}$ by $q_{i}$. Also, we denote $\psi_{e}=\left(A_{\psi_{e}}, t_{\psi_{e}}\right)$. Then (7.9) is rewritten as

$$
\left\langle A_{i} q_{i}+p_{i}-\left(A_{\psi_{e}} A_{j} q_{j}+A_{\psi_{e}} p_{j}+t_{\psi_{e}}\right), A_{i} q_{i}+p_{i}-\left(A_{\psi_{e}} A_{j} q_{j}+A_{\psi_{e}} p_{j}+t_{\psi_{e}}\right)\right\rangle=\ell_{e} .
$$

To analyze infinitesimal motions of bodies under bar-constraints, we take the derivative with respect to $\left(A_{v}, p_{v}\right)$ for $v \in V$, leading to

$$
\left\langle A_{i} q_{i}+p_{i}-\left(A_{\psi_{e}} A_{j} q_{j}+A_{\psi_{e}} p_{j}+t_{\psi_{e}}\right), \dot{A}_{i} q_{i}+\dot{p}_{i}-\left(A_{\psi_{e}} \dot{A}_{j} q_{j}+A_{\psi_{e}} \dot{p}_{j}\right)\right\rangle=0
$$

Without loss of generality, we may take $\left(A_{v}, p_{v}\right)=\left(I_{d}, 0\right)$ for all $v \in V$. Then, $\dot{A}_{v}$ is a $d \times d$ skew-symmetric matrix over $\mathbb{R}^{d}$ since the tangent space of $\mathcal{O}\left(\mathbb{R}^{d}\right)$ at the identity consists of skew-symmetric matrices. Therefore, (7.10) is a linear equation of variables $\left(\dot{A}_{i}, \dot{p}_{i}\right)$ and $\left(\dot{A}_{j}, \dot{p}_{j}\right)$ written by

$$
\left\langle q_{i}-\left(A_{\psi_{e}} q_{j}+t_{\psi_{e}}\right), \dot{A}_{i} q_{i}+\dot{p}_{i}-\left(A_{\psi_{e}} \dot{A}_{j} q_{j}+A_{\psi_{e}} \dot{p}_{j}\right)\right\rangle=0 .
$$

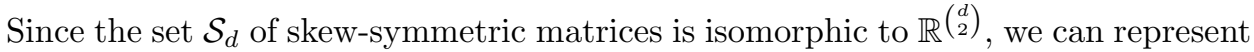

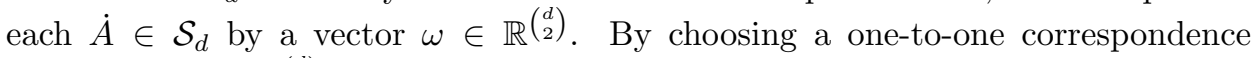
between $\mathcal{S}_{d}$ and $\mathbb{R}^{\left(\begin{array}{l}d \\ 2\end{array}\right)}$ in an appropriate manner, we have

$$
\langle h, \dot{A} q\rangle=\langle h \wedge q, \omega\rangle
$$

for any $h, q \in \mathbb{R}^{d}$ and any $\dot{A} \in \mathcal{S}_{d}$ corresponding to $\omega$. Therefore, replacing $\dot{A}_{i}$ and

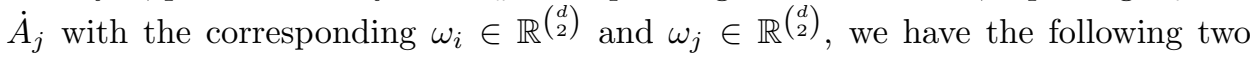
relations to simplify (7.11):

$$
\begin{aligned}
\left\langle q_{i}-A_{\psi_{e}} q_{j}-t_{\psi_{e}}, \dot{A}_{i} q_{i}\right\rangle & =\left\langle\left(q_{i}-A_{\psi_{e}} q_{j}-t_{\psi_{e}}\right) \wedge q_{i}, \omega_{i}\right\rangle \\
& =-\left\langle\left(A_{\psi_{e}} q_{j}+t_{\psi_{e}}\right) \wedge q_{i}, \omega_{i}\right\rangle, \\
\left\langle q_{i}-A_{\psi_{e}} q_{j}-t_{\psi_{e}}, A_{\psi_{e}} \dot{A}_{i} q_{j}\right\rangle & =\left\langle\left(A_{\psi_{e}}^{-1}\left(q_{i}-A_{\psi_{e}} q_{j}-t_{\psi_{e}}\right)\right) \wedge q_{j}, \omega_{j}\right\rangle \\
& =\left\langle\left(A_{\psi_{e}}^{-1}\left(q_{i}-t_{\psi_{e}}\right)\right) \wedge q_{j}, \omega_{j}\right\rangle .
\end{aligned}
$$

Thus, (7.11) can be written by

(7.12) $\left\langle q_{i}-\psi_{e} q_{j}, \dot{p}_{i}\right\rangle-\left\langle\psi_{e}^{-1} q_{i}-q_{j}, \dot{p}_{j}\right\rangle-\left\langle\left(\psi_{e} q_{j}\right) \wedge q_{i}, \omega_{i}\right\rangle-\left\langle\left(\psi_{e}^{-1} q_{i}\right) \wedge q_{j}, \omega_{j}\right\rangle=0$.

The pair $\left(\omega_{i}, \dot{p}_{i}\right)$ is conventionally called an infinitesimal motion (or a screw motion) of body $i$, and the set of all infinitesimal motions of each body forms a $\left(\begin{array}{c}d+1 \\ 2\end{array}\right)$ dimensional linear space, which can be identified with $\bigwedge^{2} \mathbb{R}^{d+1}$.

Thus, our problem is formulated as follows. For a $\Gamma$-gain graph $(G, \psi)$ and $q_{e, i}, q_{e, j} \in \mathbb{R}^{d}$ for each $e=(i, j) \in E$, an infinitesimal motion is defined by $s: i \in$ $V \mapsto s_{i}=\left(\omega_{i}, \dot{p}_{i}\right) \in \bigwedge^{2} \mathbb{R}^{d+1}$ satisfying

$\left\langle q_{e, i}-\psi_{e} q_{e, j}, \dot{p}_{i}\right\rangle-\left\langle\psi_{e}^{-1} q_{e, i}-q_{e, j}, \dot{p}_{j}\right\rangle-\left\langle\left(\psi_{e} q_{e, j}\right) \wedge q_{e, i}, \omega_{i}\right\rangle-\left\langle\left(\psi_{e}^{-1} q_{e, i}\right) \wedge q_{e, j}, \omega_{j}\right\rangle=0$

for all $e=(i, j) \in E$, and we are asked to compute the dimension of the space of infinitesimal motions. 
(7.13) can be further simplified. To see this, let $\rho: \Gamma \rightarrow G L\left(\mathbb{R}^{d}\right)$ be the linear representation of $\Gamma$ by augmented $(d+1) \times(d+1)$-matrices, i.e., $\rho(\gamma)=\left(\begin{array}{cc}A_{\gamma} & t_{\gamma} \\ 0 & 1\end{array}\right)$ for $\gamma=\left(A_{\gamma}, t_{\gamma}\right) \in \Gamma$. Also, for $q \in \mathbb{R}^{d}$, denote $\bar{q}=\left(\begin{array}{l}q \\ 1\end{array}\right) \in \mathbb{R}^{d+1}$. Since $\bar{q}_{1} \wedge \bar{q}_{2}=$ $\left(q_{1} \wedge q_{2}, q_{1}-q_{2}\right)$ for any $q_{1}, q_{2} \in \mathbb{R}^{d}$, if we denote the pair $\left(\omega_{j}, \dot{p}_{j}\right)$ by $s_{j} \in \bigwedge^{2} \mathbb{R}^{d+1}$, (7.13) becomes

$$
\left\langle\bar{q}_{e, i} \wedge \rho\left(\psi_{e}\right) \bar{q}_{e, j}, s_{i}\right\rangle-\left\langle\rho\left(\psi_{e}\right)^{-1} \bar{q}_{e, i} \wedge \bar{q}_{e, j}, s_{j}\right\rangle=0 .
$$

We may also replace $\bar{q}_{e, i}$ by $\rho\left(\psi_{e}\right) \bar{q}_{e, i}$, leading to a system of linear equations of $s: i \in V \mapsto s_{i} \in \bigwedge^{2} \mathbb{R}^{d+1}$,

$$
\left\langle\rho\left(\psi_{e}\right) \bar{q}_{e, i} \wedge \rho\left(\psi_{e}\right) \bar{q}_{e, j}, s_{i}\right\rangle-\left\langle\bar{q}_{e, i} \wedge \bar{q}_{e, j}, s_{j}\right\rangle=0 \quad \forall e=(i, j) \in E .
$$

Define $\hat{x}_{e, \psi}^{(2)}$ by

$$
\hat{x}_{e, \psi}^{(2)}(v)= \begin{cases}-\rho\left(\psi_{e}\right) \bar{q}_{e, i} \wedge \rho\left(\psi_{e}\right) \bar{q}_{e, j} & \text { if } v=i, \\ \bar{q}_{e, i} \wedge \bar{q}_{e, j} & \text { if } v=j, \\ 0 & \text { otherwise }\end{cases}
$$

for a nonloop edge $e=(i, j)$, and for each loop attached to a vertex $i$,

$$
\hat{x}_{e, \psi}^{(2)}(v)= \begin{cases}\rho\left(\psi_{e}\right) \bar{q}_{e, i} \wedge \rho\left(\psi_{e}\right) \bar{q}_{e, j}-\bar{q}_{e, i} \wedge \bar{q}_{e, j} & \text { if } v=i, \\ 0 & \text { otherwise. }\end{cases}
$$

Observe that $s$ is a solution of (17.14) if and only if $s$ is in the orthogonal complement of $\operatorname{span}\left\{\hat{x}_{e, \psi}^{(2)} \mid e \in E\right\}$. However, since $\hat{x}_{e, \psi}^{(2)}$ is a special case of (7.5), (7.6) given in the last subsection, we can apply Theorem 7.1 to compute the exact value of $\operatorname{dim}_{\mathbb{R}}\left\{\hat{x}_{e, \psi}^{(2)} \mid e \in E\right\}$ if a bar-configuration $q$ is generic. (Although the last coordinate is restricted to 1 in each $\bar{q}_{e, i}$, we can still apply Theorem 7.1 as $\operatorname{dim}_{\mathbb{R}}\left\{\hat{x}_{e, \psi}^{(2)} \mid e \in E\right\}$ is invariant up to scalar multiples of $\bar{q}_{e, i}$.) In terms of the infinitesimal rigidity of $\Gamma$-symmetric body-bar frameworks, we proved the following.

Theorem 7.2. Let $\Gamma$ be a discrete point group or a space group with representation $\rho: \gamma \mapsto\left(\begin{array}{cc}A_{\gamma} & t_{\gamma} \\ 0 & 1\end{array}\right) \in G L\left(\mathbb{R}^{d+1}\right)$, and let $H$ be a $\Gamma$-symmetric graph such that $\Gamma$ freely acts on $E(H)$. Then, for almost all body-configurations $B$ and barconfigurations $q$, the $\Gamma$-symmetric body-bar framework $(H, B, q)$ is symmetry-forced infinitesimally rigid if and only if the quotient $\Gamma$-gain graph contains an edge subset $I$ satisfying the following:

- $|I|=D|V|-D+\operatorname{dim}_{\mathbb{R}}\left\{\operatorname{image}\left(I_{D}-\rho^{(2)}(\gamma)\right) \mid \gamma \in \Gamma\right\}$;

- for any $F \subseteq I$,

$$
|F| \leq D|V(F)|-D c(F)+\sum_{X \in C(F)} \operatorname{dim}_{\mathbb{R}}\left\{\operatorname{image}\left(I_{D}-\rho^{(2)}(\gamma)\right) \mid \gamma \in\langle X\rangle\right\},
$$

where $D=\left(\begin{array}{c}d+1 \\ 2\end{array}\right)$ and $\rho^{(2)}: \Gamma \rightarrow G L\left(\bigwedge^{2} \mathbb{R}^{d+1}\right)$ is a linear representation of $\Gamma$ defined by $\rho^{(2)}(\gamma)\left(v_{1} \wedge v_{2}\right)=\rho(\gamma) v_{1} \wedge \rho(\gamma) v_{2}$ for $v_{1}, v_{2} \in \mathbb{R}^{d+1}$ and $\gamma \in \Gamma$.

As a special case when $\Gamma$ is a group of translations, Theorem 7.2 verifies a conjecture by Ross [30. 


\section{Generalization of Lift matroids}

In [45], Zaslavsky also introduced another matroid of gain graphs, called lift matroids. This matroid is a special case of elementary lifts of graphic matroids (see, e.g., 27] for elementary lifts). It was shown by Zaslavsky [4] that a lift matroid is representable over $\mathbb{F}$ if the underlying group is isomorphic to an additive subgroup of $\mathbb{F}$. In this section, we shall propose an extension of lift matroids.

8.1. Lift matroids. Let $(G=(V, E), \psi)$ be a $\Gamma$-gain graph. In the lift matroid $\mathbf{L}(G, \psi), F \subseteq E$ is independent if and only if there is at most one cycle which is unbalanced if it exists [45]. Therefore, if we define $\ell_{\Gamma}: 2^{E} \rightarrow \mathbb{Z}$ by

$$
\ell_{\Gamma}(F)=|V(F)|-c(F)+\alpha_{\Gamma}(F) \quad(F \subseteq E),
$$

where $\alpha_{\Gamma}$ is as defined in (4.2), then $\ell_{\Gamma}$ is the rank function of $\mathbf{L}(G, \psi)$.

Suppose that $\Gamma$ is an additive subgroup of $\mathbb{F}$. We shall prepare a special new element $*$ not contained in $V$, and consider a linear representation given by $e \in$ $E \mapsto L_{e} \subseteq \mathbb{F}^{V \cup\{*\}}$ with

$$
L_{e}=\left\{\begin{array}{l|l}
x \in \mathbb{F}^{V \cup\{*\}} & \begin{array}{l}
x(i)+x(j)=0, \\
\psi(e) x(i)+x(*)=0, \\
x(V \backslash\{i, j\})=0
\end{array}
\end{array}\right\} .
$$

This gives a linear representation of $\mathbf{L}(G, \psi)$, called the canonical representation of $\mathbf{L}(G, \psi)$ [47, Theorem 4.1]. It is also known that any representation of $\mathbf{L}\left(K_{n}^{\bullet}, \psi^{\bullet}\right)$ is of this form (see [47, §4] for more detail).

8.2. Generalized lift matroids. The idea of our extension of lift matroids is the same as the case of frame matroids; instead of $\alpha_{\Gamma}(F)$, we consider a submodular function over $\Gamma$.

Suppose that $(G=(V, E), \psi)$ is a $\Gamma$-gain graph with an abelian group $\Gamma$. We consider a symmetric polymatroidal function $\mu: 2^{\Gamma} \rightarrow \mathbb{R}_{+}$over $\Gamma$ (see $\$ 4.2$ for the definition).

For $F \subseteq E$, we define $\langle\langle F\rangle\rangle$ by

$$
\langle\langle F\rangle\rangle=\left\langle\psi(W) \mid W \in \pi_{1}(F, v), v \in V\right\rangle .
$$

Since $\Gamma$ is abelian, if $F$ is connected, $\langle\langle F\rangle\rangle=\langle F\rangle_{v}$ for any $v \in V(F)$ by Proposition 2.1 We define $\ell_{\mu}: 2^{E} \rightarrow \mathbb{R}$ by

$$
\ell_{\mu}(F)=|V(F)|-c(F)+\mu\langle\langle F\rangle\rangle \quad(F \subseteq E),
$$

where $\mu\langle\langle F\rangle\rangle$ is an abbreviation of $\mu(\langle\langle F\rangle\rangle)$. As in Theorem 4.1, we have the following.

Theorem 8.1. Let $(G=(V, E), \psi)$ be a $\Gamma$-gain graph with an abelian group $\Gamma$, and let $\mu$ be a symmetric polymatroidal function over $\Gamma$. If $\mu(\gamma) \leq 1$ for every $\gamma \in \Gamma$, then $\ell_{\mu}$ is monotone submodular.

Proof. For each $X \subseteq E$ and $e=(i, j) \in E \backslash X$, let $\Delta(X, e)=\ell_{\mu}(X \cup\{e\})-\ell_{\mu}(X)$, and denote by $X_{i}$ and $X_{j}$ the connected components of $X$ for which $i \in V\left(X_{i}\right)$ and $j \in V\left(X_{j}\right)$, each of which is an empty set if such a component does not exist. By a simple calculation, we have the following relation:

$$
\Delta(X, e)= \begin{cases}\mu\langle\langle X \cup\{e\}\rangle\rangle-\mu\langle\langle X\rangle\rangle & \text { if } e \text { is a loop or } X_{i}=X_{j} \neq \emptyset, \\ \mu\langle\langle X \cup\{e\}\rangle\rangle+1-\mu\langle\langle X\rangle\rangle & \text { otherwise. }\end{cases}
$$


However, since $\Gamma$ is abelian, it can be easily checked that $\langle\langle X \cup\{e\}\rangle\rangle=\langle\langle X\rangle\rangle$ if the later condition holds in (8.3). Therefore, we actually have

$$
\Delta(X, e)= \begin{cases}\mu\langle\langle X \cup\{e\}\rangle\rangle-\mu\langle\langle X\rangle\rangle & \text { if } e \text { is a loop or } X_{i}=X_{j} \neq \emptyset, \\ 1 & \text { otherwise. }\end{cases}
$$

By (8.4) and the monotonicity of $\mu$ over $\Gamma, \Delta(X, e) \geq 0$. Thus, $\ell_{\mu}$ is monotone.

To see the submodularity, we claim the following.

Claim 8.2. Let $X \subseteq E, e=(i, j) \in E \backslash X$, and $F$ a maximal forest in $X$. Suppose that $\psi(e)$ is the identity for $e \in F$. Then,

$$
\Delta(X, e)= \begin{cases}\mu(\{\psi(f) \mid f \in X \cup\{e\}\})-\mu(\{\psi(f) \mid f \in X\}) & \text { if } e \text { is a loop or } \\ & X_{i}=X_{j} \neq \emptyset, \\ 1 & \text { otherwise. }\end{cases}
$$

Moreover, $\Delta(X, e) \leq 1$.

Proof. By Proposition 2.4,

$$
\langle\langle X\rangle\rangle=\langle\psi(f) \mid f \in X\rangle \quad \text { and } \quad\langle\langle X \cup\{e\}\rangle\rangle=\langle\psi(f) \mid f \in X \cup\{e\}\rangle .
$$

By the invariance of $\mu$ under taking closure, putting (8.5) into (8.4), we obtain the relation of the statement.

To see $\Delta(X, e) \leq 1$, observe that if $e$ is a loop or $X_{i}=X_{j} \neq \emptyset$ holds, then $\Delta(X, e)=\mu(\{\psi(f) \mid f \in X \cup\{e\}\})-\mu(\{\psi(f) \mid f \in X\}) \leq \mu(\psi(e))-\mu(\emptyset) \leq 1$, where the second inequality follows from the submodularity of $\mu$ over $\Gamma$ and the third one follows from $\mu(\psi(e)) \leq 1$ and $\mu(\emptyset)=0$.

To see the submodularity of $\ell_{\mu}$, let us check $\Delta(X, e) \geq \Delta(Y, e)$ for any $X \subseteq$ $Y \subseteq E$ and $e \in E \backslash Y$. Since $\Delta(Y, e) \leq 1$ by Claim 8.2 it suffices to consider the case when $\Delta(X, e)<1$; i.e., $e$ is a loop or $X_{i}=X_{j} \neq \emptyset$. If $X_{i}=X_{j} \neq \emptyset$, then $Y_{i}=Y_{j} \neq \emptyset$ as well. Hence, $\Delta(X, e) \geq \Delta(Y, e)$ directly follows from Claim 8.2 and the submodularity (4.3) of $\mu$ over $\Gamma$.

As in the case of gain matroids, let us focus on rational functions $\mu$, i.e., $\mu: 2^{\Gamma} \rightarrow$ $\left\{0, \frac{k}{d}, \ldots, \frac{d-1}{d} k, k\right\}$ for some positive integers $d$ and $k$. Then, $d \ell_{\mu}$ is a normalized integer-valued monotone submodular function, and hence $\left(E, d \ell_{\mu}\right)$ is a polymatroid.

Example 8.1. Let us consider $\mathbb{Z}^{d}$-gain graph $(G=(V, E), \psi)$. If we define $\mu$ by $\mu(X)=\operatorname{dim}_{\mathbb{R}} X$ for $X \subseteq \mathbb{Z}^{d}$, then $\mu$ is a symmetric polymatroidal function. Hence, $\ell_{\mu}(F)=|V(F)|-c(F)+\mu\langle\langle F\rangle\rangle$ is monotone submodular. Since $\ell_{\mu}(e) \leq 1$ for $e \in E$, $\ell_{\mu}$ is indeed a rank function of a matroid on $E$.

Example 8.2. Let us consider $\mathbb{Z}^{d}$-gain graph $(G=(V, E), \psi)$, again. Define $\mu$ by $\mu(X)=\operatorname{dim}_{\mathbb{R}}\left\{\alpha \otimes \gamma \mid \alpha \in \mathbb{R}^{d}, \gamma \in X\right\} / d$ for $X \subseteq \mathbb{Z}^{d}$, where $\alpha \otimes \gamma$ denotes the tensor product of $\alpha$ and $\gamma$. Then, $\mu$ is a symmetric polymatroidal function with $\mu(\gamma) \leq 1$ for every $\gamma \in \Gamma$. Therefore, $d \ell_{\mu}$ is monotone submodular. Actually, the resulting polymatroid is just the sum of $d$ copies of the matroid given in Example 8.1

Remark 8.3. Note that lifting matroids can be defined on $\Gamma$-gain graphs with any group $\Gamma$, whereas we assumed in the above extension that $\Gamma$ is abelian. In fact, Theorem 8.1 holds even for nonabelian group $\Gamma$ if $\mu\langle\langle\cdot\rangle\rangle$ is invariant under switch operations, which is the case of lifting matroids. 
8.3. Linear representations of generalized lift matroids. We now give an extension of the canonical representation of $\mathbf{L}(G, \psi)$. Let $(G, \psi)$ be a $\Gamma$-gain graph, and suppose that $\Gamma$ is an additive subgroup of a vector space $\mathbb{F}^{t}$ over $\mathbb{F}$.

For a bilinear map $b: \mathbb{F}^{d} \times \mathbb{F}^{t} \rightarrow \mathbb{F}^{k}$, we define $\mu_{b}: 2^{\Gamma} \rightarrow \mathbb{Z}$ as follows:

$$
\mu_{b}(X)=\operatorname{dim}_{\mathbb{F}}\left\{b(\alpha, \gamma) \mid \alpha \in \mathbb{F}^{d}, \gamma \in X\right\} \quad(X \subseteq \Gamma) .
$$

Then, it is easy to check that $\mu_{b}$ is a symmetric polymatroid function over $\Gamma$. Also, for any $\gamma \in \Gamma$, we have $\mu_{b}(\gamma) \leq d$. Therefore, by Theorem 8.1, the following function $f_{b}$ induces a polymatroid on $E$ :

$$
f_{b}(F)=d|V(F)|-d c(F)+\mu_{b}\langle\langle F\rangle\rangle \quad(F \subseteq E) .
$$

For example, if setting $b: \mathbb{F} \times \mathbb{F}^{d} \rightarrow \mathbb{F}^{d}$ to be $b:(\alpha, \gamma) \mapsto \alpha \gamma$, we have the case of Example 8.1.

We now show a linear representation of the (poly)matroid induced by $f_{b}$. With each edge $e=(i, j) \in E$, we associate a linear space

$$
L_{e, \psi}=\left\{\begin{array}{l|l}
x \in\left(\mathbb{F}^{d}\right)^{V} \oplus \mathbb{F}^{k} & \begin{array}{l}
x(i)+x(j)=0, \\
b(x(i), \psi(e))+x(*)=0, \\
x(V \backslash\{i, j\})=0
\end{array}
\end{array}\right\}
$$

if $e$ is not a loop and

$$
L_{e, \psi}=\left\{x \in\left(\mathbb{F}^{d}\right)^{V} \oplus \mathbb{F}^{k} \mid \exists \alpha \in \mathbb{F}^{d:}: \begin{array}{l}
x(*)=-b(\alpha, \psi(e)), \\
x(V)=0
\end{array}\right\}
$$

if $e$ is a loop, where $\left(\mathbb{F}^{d}\right)^{V} \oplus \mathbb{F}^{k}$ is an abbreviation of $\left(\mathbb{F}^{d}\right)^{V} \oplus\left(\mathbb{F}^{k}\right)^{\{*\}}$ used throughout subsequent discussions.

We consider a linear polymatroid induced on $\left\{L_{e, \psi} \mid e \in E\right\}$. Clearly, it depends on $\psi$, but as in Lemma 5.2 the rank of the polymatroid is invariant up to equivalence.

Lemma 8.3. Let $\psi$ and $\psi^{\prime}$ be equivalent gain functions. Then, $\operatorname{dim}_{\mathbb{F}}\left\{L_{e, \psi} \mid e \in E\right\}$ $=\operatorname{dim}_{\mathbb{F}}\left\{L_{e, \psi^{\prime}} \mid e \in E\right\}$.

Proof. It is sufficient to show that the dimension is invariant from any switch operation.

Suppose that $\psi^{\prime}$ is obtained from $\psi$ by a switch operation at $v$ with $\gamma \in \Gamma$. We may assume that all edges incident to $v$ are oriented to $v$. Then, $\psi^{\prime}(e)=\psi(e)-\gamma$ if $e$ is incident to $v$; otherwise $\psi^{\prime}(e)=\psi(e)$. Note that since $\Gamma$ is abelian, $\psi^{\prime}(e)=\psi(e)$ for any loop $e$.

Consider a bijective linear transformation $T:\left(\mathbb{F}^{d}\right)^{V} \oplus \mathbb{F}^{k} \rightarrow\left(\mathbb{F}^{d}\right)^{V} \oplus \mathbb{F}^{k}$ defined by $T(x)(w)=x(w)$ for $w \in V$ and $T(x)(*)=b(x(v), \gamma)+x(*)$ for the special vertex *. We then have

$$
\begin{aligned}
T L_{e, \psi} & =\left\{\begin{array}{l}
T(x) \in\left(\mathbb{F}^{d}\right)^{V} \oplus \mathbb{F}^{k} \mid \begin{array}{l}
b(x(i), \psi(e))+x(*)=0, \\
x(i)+x(j)=0, \\
x(V \backslash\{i, j\})=0
\end{array}
\end{array}\right\} \\
& =\left\{\begin{array}{ll}
y \in\left(\mathbb{F}^{d}\right)^{V} \oplus \mathbb{F}^{k} & \begin{array}{l}
b(y(i), \psi(e))-b(y(v), \gamma)+y(*)=0, \\
y(i)+y(j)=0, \\
y(V \backslash\{i, j\})=0
\end{array}
\end{array}\right\} .
\end{aligned}
$$

Since $b(y(v), \gamma)=0$ if $v \neq i, j$, we have $b(y(i), \psi(e))-b(y(v), \gamma)=b\left(y(i), \psi^{\prime}(e)\right)$ for any $e=(i, j) \in E$. Thus, $T L_{e, \psi}=L_{e, \psi^{\prime}}$, implying the lemma. 
The following theorem is a counterpart of Theorem 5.3. whose proof is almost identical.

Theorem 8.4. Let $(G=(V, E), \psi)$ be a $\Gamma$-gain graph with an additive subgroup $\Gamma$ of $\mathbb{F}^{t}$. Define $f_{b}$ and $L_{e, \psi}$ as above. Then,

$$
f_{b}(E)=\operatorname{dim}_{\mathbb{F}}\left\{L_{e, \psi} \mid e \in E\right\} .
$$

Proof. Let $T$ be a maximal forest in $E$. By Proposition 2.3 and Lemma 8.3 we may assume that $\psi(e)=0$ for $e \in T$. Since $\Gamma$ is abelian, Proposition 2.1 and Proposition 2.4 imply that $\langle\langle E\rangle\rangle=\langle\psi(e) \mid e \in E \backslash T\rangle$, and hence

$$
f_{b}(E)=d|V(E)|-d c(E)+\operatorname{dim}_{\mathbb{F}}\left\{b(\alpha, \psi(e)) \mid \alpha \in \mathbb{F}^{d}, e \in E \backslash T\right\} .
$$

Let $L_{T}=\operatorname{span}\left\{L_{e, \psi} \mid e \in T\right\}$. By Lemma 5.1, it follows that (i) $\operatorname{dim}_{\mathbb{F}} L_{T}=$ $d|V(E)|-d c(E)$ and (ii) each quotient space $L_{e, \psi} / L_{T}$ for $e \in E \backslash T$ is written by

$$
\left\{x+L_{T} \mid \exists \alpha \in \mathbb{F}^{d}: b(\alpha, \psi(e))+x(*)=0, x(V)=0\right\},
$$

which is isomorphic to $\left\{b(\alpha, \psi(e)) \mid \alpha \in \mathbb{F}^{d}\right\}$. Therefore,

$$
\begin{aligned}
\operatorname{dim}_{\mathbb{F}}\left\{L_{e, \psi} \mid e \in E\right\} & =\operatorname{dim}_{\mathbb{F}} L_{T}+\operatorname{dim}_{\mathbb{F}}\left\{L_{e, \psi} / L_{T} \mid e \in E \backslash T\right\} \\
& =d|V(E)|-d c(E)+\operatorname{dim}_{\mathbb{F}}\left\{b(\alpha, \psi(e)) \mid \alpha \in \mathbb{F}^{d}, e \in E \backslash T\right\} \\
& =f_{b}(E) .
\end{aligned}
$$

Let $(G, \psi)$ be a $\Gamma$-gain graph. With each $e=(i, j) \in E(G)$, we associate a vector $y_{e, \psi}$ from $L_{e, \psi}$ so that $\left\{y_{e, \psi} \mid e \in E(G)\right\}$ is in generic position by extending the underlying field to $\mathbb{K}^{d}$. The following is an immediate consequence of Theorem 3.1 and Theorem 8.4 .

Corollary 8.5. Let $(G, \psi)$ be a $\Gamma$-gain graph with an additive subgroup $\Gamma$ of $\mathbb{F}^{t}$. Let $b: \mathbb{F}^{d} \times \mathbb{F}^{t} \rightarrow \mathbb{F}^{k}$ be a bilinear map. Then, $\left\{y_{e, \psi} \mid e \in E(G)\right\}$ is linearly independent in $\mathbb{K}^{d}$ if and only if for any $F \subseteq E$,

$$
|F| \leq d|V(F)|-d c(F)+\operatorname{dim}_{\mathbb{F}}\left\{b(\alpha, \gamma) \mid \alpha \in \mathbb{F}^{d}, \gamma \in\langle\langle F\rangle\rangle\right\} .
$$

8.4. Applications. Let $(G, \psi)$ be a $\mathbb{Z}^{d}$-gain graph, and let us define a bilinear map $b: \mathbb{R}^{d} \times \mathbb{R}^{d} \rightarrow \mathbb{R}^{d^{2}}$ by $b(\alpha, \gamma)=\alpha \otimes \gamma$. For each $e=(i, j) \in E(G)$, we shall associate a vector $y_{e, \psi} \in\left(\mathbb{R}^{d}\right)^{V} \oplus \mathbb{R}^{d^{2}}$ with

$$
y_{e, \psi}(v)= \begin{cases}\alpha_{e} & \text { if } v=i \\ -\alpha_{e} & \text { if } v=j \\ -\alpha_{e} \otimes \psi(e) & \text { if } v=* \\ 0 & \text { otherwise }\end{cases}
$$

such that the set of all coordinates of $\alpha_{e}(e \in E(G))$ is algebraically independent over $\mathbb{Q}$. By Corollary 8.5. $\left\{y_{e, \psi} \mid e \in E(G)\right\}$ is linearly independent if and only if for any $F \subseteq E$,

$$
|F| \leq d|V(F)|-d c(F)+d \operatorname{dim}_{\mathbb{R}}\{\gamma \mid \gamma \in\langle\langle F\rangle\rangle\} .
$$

As in $\sqrt{6}$, it is easy to check that the restriction of $\left\{L_{e, \psi} \mid e \in E\right\}$ to a generic hyperplane gives rise to the orbit rigidity matrix of a $\mathbb{Z}^{2}$-symmetric framework (called a periodic framework) when $d=2$ or to the linear representation of the $\mathbb{Z}^{d}$-symmetric parallel redrawing polymatroid of a $\mathbb{Z}^{d}$-symmetric framework for 
general dimension $d$. This implies that the independence in the associated linear (poly)matroid is characterized by the following counting condition. For any nonempty $F \subseteq E(G)$,

$$
|F| \leq d|V(F)|-d c(F)+d \operatorname{dim}_{\mathbb{R}}\{\gamma \mid \gamma \in\langle\langle F\rangle\rangle\}-1 .
$$

This is an alternative proof of results by Malestein and Theran 22 for $d=2$.

\section{TOWARD UNIFIED MATROIDS}

Although we have no clear idea of how to unify the extension of frame matroids and that of lift matroids via their rank functions, the canonical representations tell us a natural approach to unify representation theory obtained so far. To see this, in this section, we shall focus on subgroups of $G L\left(\mathbb{F}^{d}\right) \ltimes \mathbb{F}^{d}$, that is, the semidirect product of $G L\left(\mathbb{F}^{d}\right)$ and $\mathbb{F}^{d}$ with product $(g, z) \cdot\left(g^{\prime}, z^{\prime}\right)=\left(g g^{\prime}, g z^{\prime}+z\right)$.

Let $\Gamma$ be a subgroup of $G L\left(\mathbb{F}^{d}\right) \ltimes \mathbb{F}^{d}$. The projection of $\Gamma$ to the first component, i.e., $(g, z) \mapsto g$, is a group homomorphism, and hence the image $\{g \mid(g, z) \in \Gamma\}$ forms a subgroup of $G L\left(\mathbb{F}^{d}\right)$, called the linear part of $\Gamma$ and denoted by $\Gamma_{1}$.

Let $(G=(V, E), \psi)$ be a $\Gamma$-gain graph with a gain function $\psi=\left(\psi_{1}, \psi_{2}\right)$, where $\psi_{1}: E \rightarrow G L\left(\mathbb{F}^{d}\right)$ and $\psi_{2}: E \rightarrow \mathbb{F}^{d}$. Also let $b: \mathbb{F}^{d} \times \mathbb{F}^{d} \rightarrow \mathbb{F}^{k}$ be a bilinear map such that $\Gamma_{1}$ is unitary with respect to $b$, i.e., $b(g x, y)=b\left(x, g^{-1} y\right)$ for any $g \in \Gamma_{1}$ and any $x, y$. Combining the ideas of $\$ 5$ and $\$ 8$, we now associate a linear subspace with each edge $e=(i, j) \in E$ as follows:

$$
U_{e, \psi}=\left\{\begin{array}{l|l}
x \in\left(\mathbb{F}^{d}\right)^{V} \oplus \mathbb{F}^{k} & \begin{array}{l}
x(i)+\psi_{1}(e) x(j)=0, \\
x(*)=-b\left(x(i), \psi_{2}(e)\right), \\
x(V \backslash\{i, j\})=0
\end{array}
\end{array}\right\}
$$

if $e$ is not a loop, and

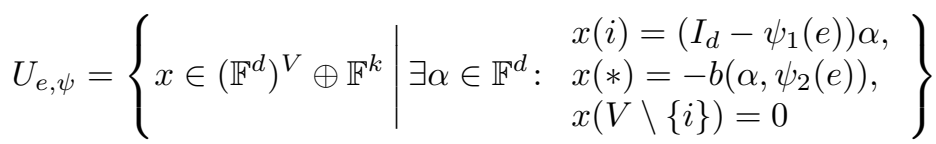

if $e$ is a loop attached to $i$.

Note that $U_{e, \psi}$ is invariant from the reorientation of $e$, as

$$
\begin{aligned}
U_{e, \psi}= & \left\{x \mid x(i)+\psi_{1}(e) x(j)=0, b\left(x(i), \psi_{2}(e)\right)+x(*)=0, x(V \backslash\{i, j\})=0\right\} \\
= & \left\{x \mid \psi_{1}(e)^{-1} x(i)+x(j)=0, b\left(-\psi_{1}(e) x(j), \psi_{2}(e)\right)+x(*)=0,\right. \\
& x(V \backslash\{i, j\})=0\} \\
=\left\{x \mid \psi_{1}(e)^{-1} x(i)+x(j)=0, b\left(x(j),-\psi_{1}(e)^{-1} \psi_{2}(e)\right)+x(*)=0,\right. & x(V \backslash\{i, j\})=0\}
\end{aligned}
$$

where $\psi(e)^{-1}=\left(\psi_{1}(e)^{-1},-\psi_{1}(e)^{-1} \psi_{2}(e)\right)$. Although $U_{e, \psi}$ depends on the choice of gain functions $\psi$, as in the previous cases, the rank of the polymatroid induced on $\left\{U_{e, \psi} \mid e \in E\right\}$ is invariant up to equivalence.

Lemma 9.1. Let $\psi$ and $\psi^{\prime}$ be equivalent gain functions. Then, $\operatorname{dim}_{\mathbb{F}}\left\{U_{e, \psi} \mid e \in E\right\}$ $=\operatorname{dim}_{\mathbb{F}}\left\{U_{e, \psi^{\prime}} \mid e \in E\right\}$.

Proof. Suppose that $\psi^{\prime}$ is obtained from $\psi$ by a switch operation at $v$ with $\gamma=$ $(g, z) \in \Gamma$. Since $U_{e, \psi}$ is invariant from reorientation of $e$, we may assume that all of the edges incident to $v$ are oriented from $v$. Then, $\psi^{\prime}(e)=\gamma \psi(e)$ if $e$ is a 
nonloop edge incident to $v, \psi^{\prime}(e)=\gamma \psi(e) \gamma^{-1}$ if $e$ is a loop incident to $v$; otherwise $\psi^{\prime}(e)=\psi(e)$.

Consider a bijective linear transformation $T:\left(\mathbb{F}^{d}\right)^{V} \oplus \mathbb{F}^{k} \rightarrow\left(\mathbb{F}^{d}\right)^{V} \oplus \mathbb{F}^{k}$ defined by, for each $x \in\left(\mathbb{F}^{d}\right)^{V} \oplus \mathbb{F}^{k}$,

$$
T(x)(w)= \begin{cases}x(w) & \text { if } w \in V \backslash\{v\}, \\ g x(v) & \text { if } w=v, \\ -b\left(x(v), g^{-1} z\right)+x(*) & \text { if } w=* .\end{cases}
$$

We then have

$$
\begin{aligned}
x(w) & =T(x)(w) \quad \text { for } w \in V \backslash\{v\}, \\
x(v) & =g^{-1} T(x)(v), \\
x(*) & =T(x)(*)+b\left(x(v), g^{-1} z\right)=T(x)(*)+b(T(x)(v), z) .
\end{aligned}
$$

Therefore, if $e$ is a nonloop edge oriented from $v$ to $j \in V$,

$$
T U_{e, \psi}=\left\{\begin{array}{l|l}
y \in\left(\mathbb{F}^{d}\right)^{V} \oplus \mathbb{F}^{k} & \begin{array}{l}
y(v)+g \psi_{1}(e) y(j)=0, \\
b\left(y(v), g \psi_{2}(e)+z\right)+y(*)=0, \\
y(V \backslash\{v, j\})=0
\end{array}
\end{array}\right\} .
$$

As $\psi^{\prime}(e)=\left(g \psi_{1}(e), g \psi_{2}(e)+z\right)$, we obtain that $T U_{e, \psi}=U_{e, \psi^{\prime}}$. Similarly, for a loop $e$ attached to $v$,

$T U_{e, \psi}$

$$
\begin{aligned}
& =\left\{y \in\left(\mathbb{F}^{d}\right)^{V} \oplus \mathbb{F}^{k} \mid \exists \alpha \in \mathbb{R}^{d}: \begin{array}{l}
y(v)=g\left(I_{d}-\psi_{1}(e)\right) \alpha \\
\left.y(*)=-b\left(\alpha,-\psi_{1}(e)^{-1} g^{-1} z+g^{-1} z+\psi_{2}(e)\right),\right\} \\
y(V \backslash\{v, j\})=0
\end{array}\right\} \\
& =\left\{y \in\left(\mathbb{F}^{d}\right)^{V} \oplus \mathbb{F}^{k} \mid \exists \alpha \in \mathbb{R}^{d}: \begin{array}{l}
y(v)=\left(I_{d}-g \psi_{1}(e) g^{-1}\right) \alpha, \\
y(*)=-b\left(\alpha,-g \psi_{1}(e)^{-1} g^{-1} z+g \psi_{2}(e)+z\right), \\
y(V \backslash\{v, j\})=0
\end{array}\right\} \\
& =U_{e, \psi^{\prime}}
\end{aligned}
$$

If $e=(i, j)$ is not incident to $v$, then we have $T(x)(i)=x(i), T(x)(j)=x(j)$, and $T(x)(*)=x(*)$ by $x(v)=0$ for any $x \in U_{e, \psi}$, and hence $T U_{e, \psi}=U_{e, \psi}=U_{e, \psi^{\prime}}$. Thus, we obtain the lemma.

By using Lemma 9.1, we can now apply the same proof as Theorem 5.3 to show a combinatorial characterization of the polymatroid induced on $\left\{U_{e, \psi} \mid e \in E\right\}$. To see this, we need new terminology. Consider $F \subseteq E$. Recall that $G[F]$ denotes the edge-induced subgraph $(V(F), F)$. By Proposition 2.3. for a maximal forest $T$ of $F$, there is an equivalent gain function $\psi_{F}^{\circ}$ to $\psi$ such that $\psi_{F}^{\circ}(e)$ is the identity for all $e \in T$. A compressed graph by $F$ is defined as a $\Gamma$-gain graph $\left(G_{F}^{\circ}, \psi_{F}^{\circ}\right)$, where $G_{F}^{\circ}$ is obtained from $G[F]$ by contracting each connected component to a single vertex, where each edge $e$ of $F$ remains in $G_{F}^{\circ}$ as a loop with the gain $\psi_{F}^{\circ}(e)$. By Proposition 2.3 and Proposition 2.4 $\left(G_{F}^{\circ}, \psi_{F}^{\circ}\right)$ is invariant from the choice of $T$ up to the equivalence of gain functions $\psi_{F}^{\circ}$.

Applying the same proof as Theorem 8.4 we now have the following result. We omit the proof, which is identical to those of Theorem 5.3 and Theorem 8.4 . 
Theorem 9.2. Let $\Gamma$ be a subgroup of $G L\left(\mathbb{F}^{d}\right) \ltimes \mathbb{F}^{d}$ and $\Gamma_{1}$ the projection of $\Gamma$ to $G L\left(\mathbb{F}^{d}\right)$. Let $(G, \psi)$ be a $\Gamma$-gain graph, and $b: \mathbb{F}^{d} \times \mathbb{F}^{d} \rightarrow \mathbb{F}^{k}$ a bilinear map such that $\Gamma_{1}$ is unitary with respect to b. Then, for any $F \subseteq E(G)$,

$$
\operatorname{dim}_{\mathbb{F}}\left\{U_{e, \psi} \mid e \in F\right\}=d|V(F)|-d c(F)+\operatorname{dim}_{\mathbb{F}}\left\{U_{e, \psi_{F}^{\circ}} \mid e \in E\left(G_{F}^{\circ}\right)\right\} .
$$

Remark 9.1. Theorem 9.2 gives a good characterization of the dimension of $\operatorname{span}\left\{U_{e, \psi} \mid e \in F\right\}$, since computing $\operatorname{dim}_{\mathbb{F}}\left\{U_{e, \psi_{F}^{\circ}} \mid e \in E\left(G_{F}^{\circ}\right)\right\}$ can be reduced to the computation of the rank of a matrix of size $(d c(F)+k) \times d|F|$. Hence, it is possible to compute $\operatorname{dim}_{\mathbb{F}}\left\{U_{e, \psi} \mid e \in F\right\}$ deterministically in polynomial time.

\section{FurTher APPLiCATIONS}

As applications of Theorem 9.2 , we shall extend the result of 96 to symmetric frameworks with crystallographic symmetry. For detailed analysis, we first review basic facts on space groups in $\$ 10.1$. In $\$ 10.2$ and $\$ 10.3$. we discuss the parallel redrawing problem and the symmetry-forced rigidity, respectively.

10.1. Space groups. Recall that a space group (or crystallographic group) $\Gamma$ is a discrete cocompact subgroup of the Euclidean group $\mathcal{E}(d)$, and each element $\left(A_{\gamma}, t_{\gamma}\right) \in \Gamma$ acts on $\mathbb{R}^{d}$ by $\left(A_{\gamma}, t_{\gamma}\right) \cdot q=A_{\gamma} q+t_{\gamma}$ for $q \in \mathbb{R}^{d}$. An element of the form $\left(I_{d}, t_{\gamma}\right)$ is called a translation. As in the previous section, let $\Gamma_{1}=\left\{A_{\gamma} \mid \gamma \in \Gamma\right\}$, the projection to the first component.

The subgroup $\mathcal{L}_{\Gamma}$ consisting of all translations in $\Gamma$ is called the lattice group of $\Gamma$, and it is known by Bieberbach's theorem that $\mathcal{L}_{\Gamma}$ is a normal subgroup of $\Gamma$ generated by $d$ linearly independent translations $t_{1}, \ldots, t_{d} \in \mathbb{R}^{d}$. The $d \times d$-matrix $B_{\Gamma}$ of the base transformation from the standard basis of $\mathbb{R}^{d}$ to $\left\{t_{1}, \ldots, t_{d}\right\}$ is called a lattice basis of $\Gamma$. (Conventionally, a lattice basis of $\mathcal{L}_{\Gamma}$ means $\left\{t_{1}, \ldots, t_{d}\right\}$ rather than $B_{\Gamma}$.) Then, $\mathcal{L}_{\Gamma}=\left\{B_{\Gamma} z \mid z \in \mathbb{Z}^{d}\right\}$.

The quotient subgroup $\mathcal{K}_{\Gamma}=\Gamma / \mathcal{L}_{\Gamma}$ is known as the point group of $\Gamma$. Since $\mathcal{K}_{\Gamma}$ acts on $\mathcal{L}_{\Gamma}$ and $\mathcal{L}_{\Gamma}$ is isomorphic to $\mathbb{Z}^{d}, \mathcal{K}_{\Gamma}$ can be represented as integral matrices. Therefore, in the subsequent discussion, $\mathcal{K}_{\Gamma}$ is regarded as a finite subgroup of $G L\left(\mathbb{Z}^{d}\right)$. Note then that $B_{\Gamma} \mathcal{K}_{\Gamma} B_{\Gamma}^{-1}=\Gamma_{1} \subseteq \mathcal{O}\left(\mathbb{R}^{d}\right)$. Indeed, using the lattice basis $B_{\Gamma}$, each element $\gamma=\left(A_{\gamma}, t_{\gamma}\right)$ of $\Gamma$ can be uniquely written by a triple $\left(K_{\gamma}, z_{\gamma}, c_{\gamma}\right) \in G L\left(\mathbb{Z}^{d}\right) \times \mathbb{Z}^{d} \times[0,1)^{d}$, where $A_{\gamma}=B_{\Gamma} K_{\gamma} B_{\Gamma}^{-1}$ and $t_{\gamma}=B_{\Gamma}\left(z_{\gamma}+c_{\gamma}\right)$. The representation in $G L\left(\mathbb{Z}^{d}\right) \times \mathbb{Z}^{d} \times[0,1)^{d}$ is sometimes called the standard form. Note that a space group $\Gamma$ is determined by the standard form of each element and the lattice basis.

By Bieberbach's theorem, two space groups $\Gamma$ and $\Gamma^{\prime}$ are isomorphic if and only if they are conjugate by an affine transformation in $\operatorname{Aff}\left(\mathbb{R}^{d}\right)$. Eliminating isometries, we focus on affine motions that change the lattice basis $B_{\Gamma}$ without changing the linear part. We hence define the space of lattices by

$$
\operatorname{Lat}(\Gamma)=\left\{B \in G L\left(\mathbb{R}^{d}\right) \mid \forall K_{\gamma} \in \mathcal{K}_{\Gamma}: B K_{\gamma} B^{-1}=B_{\Gamma} K_{\gamma} B_{\Gamma}^{-1}\left(=A_{\gamma}\right)\right\},
$$

and here we say that $\Gamma$ and $\Gamma^{\prime}$ are equivalent if $B_{\Gamma^{\prime}} \in \operatorname{Lat}(\Gamma)$. It is convenient to consider a slightly larger set

$$
\overline{\operatorname{Lat}}(\Gamma)=\left\{B \in \mathbb{R}^{d \times d} \mid \forall K_{\gamma} \in \mathcal{K}_{\Gamma}: B K_{\gamma}=A_{\gamma} B\right\} .
$$

Then, $\overline{\operatorname{Lat}}(\Gamma)$ is a linear space and $\operatorname{Lat}(\Gamma)$ is a dense open subset of $\overline{\operatorname{Lat}}(\Gamma)$. 
10.2. Parallel redrawing with space group symmetry. Let us move to the $\Gamma$-symmetric parallel redrawing problem for a space group $\Gamma$ with linear part $\Gamma_{1}$. Let $\mathcal{L}_{\Gamma}$ be the lattice group of $\Gamma$ with a basis $B_{\Gamma} \in G L\left(\mathbb{R}^{d}\right)$. Each element $\gamma \in \Gamma$ is denoted by $\left(A_{\gamma}, t_{\gamma}\right) \in \mathcal{O}\left(\mathbb{R}^{d}\right) \ltimes \mathbb{R}^{d}$, but we also use the standard form $\left(K_{\gamma}, z_{\gamma}, c_{\gamma}\right) \in$ $G L\left(\mathbb{Z}^{d}\right) \times \mathbb{Z}^{d} \times[0,1)^{d}$, where $A_{\gamma}=B_{\Gamma} K_{\gamma} B_{\Gamma}^{-1}$ and $t_{\gamma}=B_{\Gamma}\left(z_{\gamma}+c_{\gamma}\right)$.

We consider a $\Gamma$-symmetric framework $(H, p)$, where $H$ is a $\Gamma$-symmetric graph and $p$ is a $\Gamma$-symmetric point-configuration. We say that $(H, q)$ is a symmetric parallel redrawing of $(H, p)$ if $(H, q)$ is a parallel redrawing of $(H, p)$ and it is $\Gamma^{\prime}$ symmetric for some equivalent space group $\Gamma^{\prime}$ to $\Gamma$.

A map $m: V(H) \rightarrow \mathbb{R}^{d}$ is called a relocation of $(H, p)$ if

$$
m(i)-m(j) \text { is parallel to } p(i)-p(j) \text { for any }\{i, j\} \in E(H) \text {. }
$$

We should mention the following two remarks. (1) Since $m$ is a vector rather than a point in the Euclidean space, only $\Gamma_{1}$ acts on the space of relocations. (2) A framework can also be relocated by deforming the underlying lattice. Thus, we say that a relocation $m$ is $\Gamma$-symmetric if there is $M \in \overline{\operatorname{Lat}}(\Gamma)$ such that $M+B_{\Gamma} \in$ Lat $(\Gamma)$ and

$$
m(\gamma v)=A_{\gamma} m(v)+M\left(z_{\gamma}+c_{\gamma}\right) \quad \forall v \in V(H), \forall \gamma \in \Gamma .
$$

The definition is justified by the following proposition.

Proposition 10.1. Let $(H, p)$ be a $\Gamma$-symmetric framework with a space group $\Gamma$. If $m$ is a $\Gamma$-symmetric relocation of $(H, p)$, then $(H, p+m)$ is a symmetric parallel redrawing of $(H, p)$. Conversely, if $(H, q)$ is a symmetric parallel redrawing, then $q-p$ is a $\Gamma$-symmetric relocation.

Proof. Suppose that $m$ is a $\Gamma$-symmetric relocation. Define $q$ by $q(v)=p(v)+m(v)$ for $v \in V(H)$. Since $m$ is a relocation, $(H, q)$ is a parallel redrawing of $(H, p)$ by (10.3). Also, since $m$ is $\Gamma$-symmetric, there exists $M \in \overline{\operatorname{Lat}}(\Gamma)$ for which $M+B_{\Gamma} \in$ $\operatorname{Lat}(\Gamma)$ and (10.4) is satisfied. Let $B=M+B_{\Gamma}$. Then, for any $v \in V(H)$ and $\gamma \in \Gamma$,

$$
\begin{aligned}
q(\gamma v) & =p(\gamma v)+m(\gamma v)=A_{\gamma} p(v)+B_{\Gamma}\left(z_{\gamma}+c_{\gamma}\right)+A_{\gamma} m(v)+M\left(z_{\gamma}+c_{\gamma}\right) \\
& =A_{\gamma}(p(v)+m(v))+B\left(z_{\gamma}+c_{\gamma}\right)=A_{\gamma} q(v)+B\left(z_{\gamma}+c_{\gamma}\right) .
\end{aligned}
$$

Since $B \in \operatorname{Lat}(\Gamma)$, this implies that $(H, q)$ is $\Gamma^{\prime}$-symmetric for an equivalent $\Gamma^{\prime}$ to $\Gamma$.

Conversely, suppose that $(H, q)$ is a symmetric parallel redrawing of $(H, p)$. Then, $(H, q)$ is $\Gamma^{\prime}$-symmetric for some equivalent $\Gamma^{\prime}$ to $\Gamma$. This means that there is $B \in \operatorname{Lat}(\Gamma)$ such that $B$ is a lattice basis of $\Gamma^{\prime}$. Setting $m=q-p$ and $M=B-B_{\Gamma}$, we see that $M \in \overline{\operatorname{Lat}}(\Gamma)$ and for any $v \in V(H)$ and $\gamma \in \Gamma$,

$$
\begin{aligned}
m(\gamma v)= & q(\gamma v)-p(\gamma v)=B K_{\gamma} B^{-1} q(v)+B\left(z_{\gamma}+c_{\gamma}\right) \\
& -\left(B_{\Gamma} K_{\gamma} B_{\Gamma}^{-1} p(v)+B_{\Gamma}\left(z_{\gamma}+c_{\gamma}\right)\right) \\
= & B_{\Gamma} K_{\gamma} B_{\Gamma}^{-1}(q(v)-p(v))+\left(B-B_{\Gamma}\right)\left(z_{\gamma}+c_{\gamma}\right) \\
= & A_{\gamma} m(v)+M\left(z_{\gamma}+c_{\gamma}\right),
\end{aligned}
$$

implying that $m$ is a $\Gamma$-symmetric relocation of $(H, p)$.

As in the case of point group symmetry, a relocation $m$ is said to be trivial if $m$ is a linear combination of translations $m_{t}$ and a dilation $m_{\mathrm{di}}$. Indeed, for any 
$t \in \bigcap_{\gamma \in \Gamma}\left(A_{\gamma}-I_{d}\right)$, translation $m_{t}$ defined by $m_{t}(v)=t$ for $v \in V(H)$ is a $\Gamma$ symmetric relocation with $M=0$; on the other hand, a dilation $m_{\mathrm{di}}$ defined by $m_{\mathrm{di}}(v)=p(v)$ for $v \in V(H)$ is also a $\Gamma$-symmetric relocation with $M=B_{\Gamma}$.

Motivated by Proposition 10.1, a $\Gamma$-symmetric $(H, p)$ is said to be symmetrically robust if all possible $\Gamma$-symmetric relocations are trivial. Let us then show that checking the robustness can be reduced to computing the rank of linear polymatroids of quotient gain graphs. We first remark that the condition for $M$ to be $M+B_{\Gamma} \in \operatorname{Lat}(\Gamma)$ can be ignored in the analysis.

Lemma 10.2. A $\Gamma$-symmetric $(H, p)$ is not symmetrically robust if and only if there are $m: V(H) \rightarrow \mathbb{R}^{d}$ and $M \in \overline{\mathrm{Lat}}(\Gamma)$ for which (10.3) and (10.4) are satisfied and $m$ is not a trivial relocation.

Proof. The sufficiency is trivial from the definition. To see the necessity, suppose there are such $m$ and $M$. Then, for any $\epsilon \in \mathbb{R}, \epsilon m$ and $\epsilon M$ satisfy (10.3) and (10.4). Moreover, if we take $\epsilon$ small enough, $B_{\Gamma}+\epsilon M \in \operatorname{Lat}(\Gamma)$ holds as $B_{\Gamma}$ is nonsingular. Therefore $\epsilon m$ is a nontrivial $\Gamma$-symmetric relocation, and hence $(H, p)$ is not symmetrically robust.

As in the finite case, we now simplify the system (10.3). Recall that each edge orbit is written by $\Gamma e=\left\{\left(\gamma i, \gamma \psi_{e} j\right) \mid \gamma \in \Gamma\right\}$ with $\psi(e)=\psi_{e}=\left(A_{\psi_{e}}, z_{\psi_{e}}+c_{\psi_{e}}\right)$. Thus, for each edge $\left(\gamma i, \gamma \psi_{e} j\right)$ in an edge orbit $\Gamma e,(10.3)$ is written by

$$
\left\langle m(\gamma i)-m\left(\gamma \psi_{e} j\right), \alpha\right\rangle=0 \quad \forall \alpha \in \mathbb{R}^{d} \text { such that }\left\langle p(\gamma i)-p\left(\gamma \psi_{e} j\right), \alpha\right\rangle=0 .
$$

These are indeed equivalent to one equation,

$$
\left\langle m(i)-m\left(\psi_{e} j\right), \alpha\right\rangle=0 \quad \forall \alpha \in \mathbb{R}^{d} \text { such that }\left\langle p(i)-p\left(\psi_{e} j\right), \alpha\right\rangle=0,
$$

which is further converted to, by (10.4),

$$
\left\langle m(i)-\left(A_{\psi_{e}} m(j)+M\left(z_{\psi_{e}}+c_{\psi_{e}}\right)\right), \alpha\right\rangle=0
$$

for all $\alpha \in \mathbb{R}^{d}$ such that $\left\langle p(i)-\left(A_{\psi_{e}} p(j)+t_{\psi_{e}}\right), \alpha\right\rangle=0$ and for some $M \in \overline{\operatorname{Lat}}(\Gamma)$.

Therefore the problem can be considered in a general $\Gamma$-gain graph $(G=$ $(V, E), \psi)$, and by Lemma 10.2 we are interested in the space of $(m, M) \in\left(\mathbb{R}^{d}\right)^{V} \oplus$ $\overline{\operatorname{Lat}}(\Gamma)$ satisfying

$$
\left\langle m(i)-\left(A_{\psi_{e}} m(j)+M\left(z_{\psi_{e}}+c_{\psi_{e}}\right)\right), \alpha\right\rangle=0
$$

for every $e=(i, j) \in E$ and every $\alpha \in \mathbb{R}^{d}$ such that $\left\langle p(i)-\left(A_{\psi_{e}} p(j)+t_{\psi_{e}}\right), \alpha\right\rangle=0$. For further analysis, we shall take a basis $B_{1}, \ldots, B_{k} \in \mathbb{R}^{d \times d}$ of $\overline{\operatorname{Lat}}(\Gamma)$, where $k=\operatorname{dim}_{\mathbb{R}} \overline{\operatorname{Lat}}(\Gamma)$. We then define a bilinear function $b_{i}: \mathbb{R}^{d} \times \mathbb{R}^{d} \rightarrow \mathbb{R}$ by

$$
b_{i}(\alpha, t)=\left\langle\alpha, B_{i} B_{\Gamma}^{-1} t\right\rangle \quad\left((\alpha, t) \in \mathbb{R}^{d} \times \mathbb{R}^{d}\right) .
$$

Then, a bilinear map $b: \mathbb{R}^{d} \times \mathbb{R}^{d} \rightarrow \mathbb{R}^{k}$ is defined by $b(\alpha, t)=\left(b_{1}(\alpha, t), \ldots, b_{k}(\alpha, t)\right)^{\top}$.

Observe that $\Gamma_{1}$ is unitary with respect to $b_{i}$. Indeed, for each $\gamma \in \Gamma$, we have $A_{\gamma} B_{\Gamma}=B_{\Gamma} K_{\gamma}$ and $A_{\gamma} B_{i}=B_{i} K_{\gamma}$ as $B_{\Gamma}, B_{i} \in \overline{\operatorname{Lat}}(\Gamma)$, and hence $b_{i}\left(A_{\gamma} \alpha, t\right)=$ $\left\langle A_{\gamma} \alpha, B_{i} B_{\Gamma}^{-1} t\right\rangle=\left\langle\alpha, A_{\gamma}^{-1} B_{i} B_{\Gamma}^{-1} t\right\rangle=\left\langle\alpha, B_{i} K_{\gamma}^{-1} B_{\Gamma}^{-1} t\right\rangle=\left\langle\alpha, B_{i} B_{\Gamma}^{-1} A_{\gamma}^{-1} t\right\rangle=$ $b_{i}\left(\alpha, A_{\gamma}^{-1} t\right)$. Hence, $\Gamma_{1}$ is also unitary with respect to $b$.

We shall associate a $(d-1)$-dimensional linear subspace $P_{e, \psi}^{\prime}(p)$ with each edge $e=(i, j) \in E$, defined by

$$
P_{e, \psi}^{\prime}(p)=U_{e, \psi} \cap\left\{x \in\left(\mathbb{R}^{d}\right)^{V} \oplus \mathbb{R}^{k} \mid\left\langle p(i)-\left(A_{\psi_{e}} p(j)+t_{\psi_{e}}\right), x(i)\right\rangle=0\right\}
$$


where $U_{e, \psi}$ is, as defined in (9.1), (9.2),

$$
U_{e, \psi}=\left\{\begin{array}{l|l}
x \in\left(\mathbb{R}^{d}\right)^{V} \oplus \mathbb{R}^{k} & \begin{array}{l}
x(i)+A_{\psi_{e}} x(j)=0 \\
b\left(x(i), t_{\psi_{e}}\right)+x(*)=0 \\
x(V \backslash\{i, j\})=0
\end{array}
\end{array}\right\}
$$

or

$$
U_{e, \psi}=\left\{x \in\left(\mathbb{R}^{d}\right)^{V} \oplus \mathbb{R}^{k} \mid \exists \alpha \in \mathbb{R}^{d}: \begin{array}{l}
x(i)=\left(I_{d}-A_{\psi_{e}}\right) \alpha, \\
\\
x(V \backslash\{i, j\})=0
\end{array}\right\}
$$

depending on whether $e$ is a nonloop or a loop, respectively.

Lemma 10.3. Let $(G=(V, E), \psi)$ be a $\Gamma$-gain graph with a space group $\Gamma$. Then, the dimension of the space of $(m, M) \in\left(\mathbb{R}^{d}\right)^{V} \oplus \overline{\operatorname{Lat}}(\Gamma)$ satisfying (10.5) is equal to

$$
d|V|+k-\operatorname{dim}_{\mathbb{R}}\left\{P_{e, \psi}^{\prime}(p) \mid e \in E\right\}
$$

where $k=\operatorname{dim}_{\mathbb{R}} \overline{\operatorname{Lat}}(\Gamma)$.

Proof. Since $\left\{B_{1}, \ldots, B_{k}\right\}$ is a basis of $\overline{\operatorname{Lat}}(\Gamma), \overline{\operatorname{Lat}}(\Gamma)$ is parameterized by $k$ parameters $a=\left(a_{1}, \ldots, a_{k}\right)^{\top} \in \mathbb{R}^{k}$ such that $\overline{\operatorname{Lat}}(\Gamma)=\left\{\sum_{1 \leq \ell \leq k} a_{\ell} B_{\ell} \mid a \in \mathbb{R}^{k}\right\}$. In other words, the space of $(m, M)$ satisfying (10.5) is isomorphic to the space of $(m, a) \in\left(\mathbb{R}^{d}\right)^{V} \oplus \mathbb{R}^{k}$ satisfying

$$
\left\langle m(i)-\left(A_{\psi_{e}} m(j)+\sum_{1 \leq \ell \leq k} a_{\ell} B_{\ell}\left(z_{\psi_{e}}+c_{\psi_{e}}\right)\right), \alpha\right\rangle=0
$$

for every $e=(i, j) \in E$ and every $\alpha \in \mathbb{R}^{d}$ with $\left\langle p(i)-\left(A_{\psi_{e}} p(j)+t_{\psi_{e}}\right), \alpha\right\rangle=0$. Observe then that $(m, a) \in\left(\mathbb{R}^{d}\right)^{V} \oplus \mathbb{R}^{k}$ satisfies (10.10) if and only if $(m, a)$ is in the orthogonal complement of $\operatorname{span}\left\{P_{e, \psi}^{\prime}(p) \mid e \in E\right\}$, because, for any $x \in P_{e, \psi}^{\prime}(p)$, we have

$$
\begin{aligned}
\langle(m, a), x\rangle & =\langle m(i), x(i)\rangle+\langle m(j), x(j)\rangle+\langle a, x(*)\rangle \\
& =\langle m(i), x(i)\rangle-\left\langle m(j), A_{\psi_{e}}^{-1} x(i)\right\rangle-\left\langle a, b\left(x(i), t_{\psi_{e}}\right)\right\rangle \\
& =\left\langle m(i)-A_{\psi_{e}} m(j), x(i)\right\rangle-\sum_{\ell} a_{\ell} b_{\ell}\left(x(i), t_{\psi_{e}}\right) \\
& =\left\langle m(i)-A_{\psi_{e}} m(j), x(i)\right\rangle-\sum_{\ell} a_{\ell}\left\langle B_{\ell}\left(z_{\psi_{e}}+c_{\psi_{e}}\right), x(i)\right\rangle \\
& =\left\langle m(i)-A_{\psi_{e}} m(j)-\sum_{\ell} a_{\ell} B_{\ell}\left(z_{\psi_{e}}+c_{\psi_{e}}\right), x(i)\right\rangle
\end{aligned}
$$

with $\left\langle p(i)-\left(A_{\psi_{e}} p(j)+t_{\psi_{e}}\right), x(i)\right\rangle=0$.

Since the set of trivial relocations forms a linear space of dimension $\left(\operatorname{dim}_{\mathbb{R}} \bigcap_{\gamma \in \Gamma} \operatorname{ker}\left(A_{\gamma}-I_{d}\right)\right)+1$, Lemmas 10.2 and 10.3 imply the following.

Corollary 10.4. Let $(H, p)$ be a $\Gamma$-symmetric framework with a space group $\Gamma$, and $(H / \Gamma, \psi)$ be the quotient $\Gamma$-gain graph of $H$. Then, $(H, p)$ is symmetrically robust if and only if

$$
\operatorname{dim}_{\mathbb{R}}\left\{P_{e, \psi}^{\prime}(p / \Gamma) \mid e \in E(H / \Gamma)\right\}=d|V / \Gamma|+k-1-\operatorname{dim}_{\mathbb{R}} \bigcap_{\gamma \in \Gamma} \operatorname{ker}\left(A_{\gamma}-I_{d}\right)
$$


10.2.1. Combinatorial characterization. By Corollary 10.4, in order to decide the robustness of frameworks, it now suffices to analyze the $\Gamma$-symmetric parallel redrawing polymatroid of a $\Gamma$-gain graph $(G=(V, E), \psi)$, which is defined as the linear polymatroid with linear representation $e \mapsto P_{e, \psi}^{\prime}(p)$. The following theorem provides a combinatorial characterization of this polymatroid.

We say that the lattice of $\Gamma$ is generic if $B_{\Gamma}$ is expressed by $B_{\Gamma}=\sum_{i=1}^{k} s_{i} B_{i}$ such that $\left\{s_{1}, \ldots, s_{k}\right\}$ is algebraically independent over $\mathbb{Q}_{\Gamma_{1}}$.

Theorem 10.5. Let $(G=(V, E), \psi)$ be a $\Gamma$-gain graph for a space group $\Gamma$ with a generic lattice and $k=\operatorname{dim}_{\mathbb{R}} \overline{\operatorname{Lat}}(\Gamma)$. Define $h_{\Gamma}: 2^{E} \rightarrow \mathbb{Z}$ by

$$
h_{\Gamma}(F)=d|V(F)|-d c(F)+\operatorname{dim}_{\mathbb{R}}\left\{U_{e, \psi_{F}^{\circ}} \mid e \in E\left(G_{F}^{\circ}\right)\right\}-1 \quad(F \subseteq E),
$$

where $\left(G_{F}^{\circ}, \psi_{F}^{\circ}\right)$ is the compressed graph of $(G, \psi)$ by $F$ (defined in 99 ). Then, for almost all $p: V \rightarrow \mathbb{R}^{d}$,

$$
\operatorname{dim}_{\mathbb{R}}\left\{P_{e, \psi}^{\prime}(p) \mid e \in E\right\}=\hat{h}_{\Gamma}(E) .
$$

In other words, the $\Gamma$-symmetric parallel redrawing polymatroid is equal to the polymatroid induced by $h_{\Gamma}$.

Proof. Let $h_{\Gamma}^{\prime}=h_{\Gamma}+1$. Note that the linear polymatroid $\mathbf{L P}(E, \Psi)$ of the linear representation $\Psi: e \mapsto U_{e, \psi}$ is a special case of those given in \$9 and hence Theorem 9.2 implies that $h_{\Gamma}^{\prime}(F)=\operatorname{dim}_{\mathbb{R}}\left\{U_{e, \psi} \mid e \in F\right\}$ for all $F \subseteq E$.

Since the lattice of $\Gamma$ is generic, the lattice basis $B_{\Gamma}$ is written by $B_{\Gamma}=\sum_{i=1}^{k} s_{i} B_{i}$, where $\left\{s_{1}, \ldots, s_{k}\right\}$ is algebraically independent over $\mathbb{Q}_{\Gamma_{1}}$. Let us take any $p$ : $V \rightarrow \mathbb{R}^{d}$ such that the set of coordinates of $p$ and $s_{1}, \ldots, s_{k}$ form an algebraically independent set over $\mathbb{Q}_{\Gamma_{1}}$. We define a hyperplane $\mathcal{H}$ of $\left(\mathbb{R}^{d}\right)^{V} \oplus \mathbb{R}^{k}$ by

$$
\mathcal{H}=\left\{x \in\left(\mathbb{R}^{d}\right)^{V} \oplus \mathbb{R}^{k} \mid \sum_{v \in V}\langle p(v), x(v)\rangle+\langle s, x(*)\rangle=0\right\}
$$

Then, observe that $P_{e, \psi}^{\prime}(p)=U_{e, \psi} \cap \mathcal{H}$ for every $e=(i, j) \in E$, since for any $x \in U_{e, \psi}$ we have

$$
\begin{aligned}
\sum_{v \in V}\langle p(v), x(v)\rangle+\langle s, x(*)\rangle \\
=\langle p(i), x(i)\rangle+\langle p(j), x(j)\rangle+\langle s, x(*)\rangle \\
=\langle p(i), x(i)\rangle+\left\langle p(j),-A_{\psi_{e}}^{-1} x(i)\right\rangle+\left\langle s,-b\left(x(i), t_{\psi_{e}}\right)\right\rangle \\
=\langle p(i), x(i)\rangle-\left\langle A_{\psi_{e}} p(j), x(i)\right\rangle-\sum_{\ell} s_{\ell}\left\langle B_{\ell}\left(z_{\psi_{e}}+c_{\psi_{e}}\right), x(i)\right\rangle \\
\quad=\left\langle p(i)-\left(A_{\psi_{e}} p(j)+t_{\psi_{e}}\right), x(i)\right\rangle,
\end{aligned}
$$

by $t_{\psi_{e}}=B\left(z_{\psi_{e}}+c_{\psi_{e}}\right)=\left(\sum_{i} s_{i} B_{i}\right)\left(z_{\psi_{e}}+c_{\psi_{e}}\right)$. Therefore, as the coordinates of $p$ and $s_{1}, \ldots, s_{k}$ form an algebraically independent set over $\mathbb{Q}_{\Gamma_{1}}$, we conclude that the $\Gamma$-symmetric parallel redrawing polymatroid of $(G, \psi)$ is obtained from $\mathbf{L P}(E, \Psi)$ 
by a Dilworth truncation. By Theorem 3.2 , we finally obtain

$$
\begin{aligned}
& \operatorname{dim}_{\mathbb{R}}\left\{P_{e, \psi}^{\prime}(p) \mid e \in E\right\} \\
& =\min \left\{\sum_{i}\left(\operatorname{dim}_{\mathbb{R}}\left\{U_{e, \psi} \mid e \in E_{i}\right\}-1\right) \mid \text { a partition }\left\{E_{1}, \ldots, E_{k}\right\} \text { of } E\right\} \\
& =\min \left\{\sum_{i}\left(h_{\Gamma}^{\prime}\left(E_{i}\right)-1\right) \mid \text { a partition }\left\{E_{1}, \ldots, E_{k}\right\} \text { of } E\right\} \\
& =\min \left\{\sum_{i} h_{\Gamma}\left(E_{i}\right) \mid \text { a partition }\left\{E_{1}, \ldots, E_{k}\right\} \text { of } E\right\} \\
& =\hat{h}_{\Gamma}(E) .
\end{aligned}
$$

Combining Corollary 10.4 and Theorem 10.5, we complete characterizing the symmetric robustness of drawings with crystallographic symmetry.

Corollary 10.6. Let $H$ be a $\Gamma$-symmetric graph with a space group $\Gamma$ with a generic lattice, and let $(G, \psi)$ be the quotient $\Gamma$-gain graph of $H$. For almost all $\Gamma$-symmetric $p: V(H) \rightarrow \mathbb{R}^{d},(H, p)$ is symmetrically robust if and only if the $\Gamma$-gain graph $(\tilde{G}, \psi)$ obtained from $(G, \psi)$ by replacing each edge $e \in E(G)$ by $d-1$ parallel copies contains an edge subset I satisfying the following counting conditions:

- $|I|=d|V|+k-1-\operatorname{dim}_{\mathbb{R}} \bigcap_{\gamma \in \Gamma} \operatorname{ker}\left(A_{\gamma}-I_{d}\right)$;

- $|F| \leq d|V(F)|-d c(F)-1+\operatorname{dim}_{\mathbb{R}}\left\{U_{e, \psi_{F}^{\circ}} \mid e \in E\left(\tilde{G}_{F}^{\circ}\right)\right\}$ for any nonempty $F \subseteq I$

where $\left(\tilde{G}_{F}^{\circ}, \psi_{F}^{\circ}\right)$ is the compressed graph of $\tilde{G}$ by $F$ and $k=\operatorname{dim}_{\mathbb{R}} \overline{\operatorname{Lat}}(\Gamma)$.

Remark 10.1. As we have remarked, the dimension of $\bigcap_{\gamma \in \Gamma} \operatorname{ker}\left(A_{\gamma}-I_{d}\right)$ is equal to $\frac{1}{\left|\Gamma_{1}\right|} \sum_{A \in \Gamma_{1}} \operatorname{trace}(A)$, and $\operatorname{dim}_{\mathbb{R}}\left\{U_{e, \psi_{F}^{\circ}} \mid e \in E\left(\tilde{G}_{F}^{\circ}\right)\right\}$ can be deterministically computed in polynomial time. Thus, Corollary 10.6 gives a good characterization of the symmetric robustness. Checking the counting condition can be deterministically done in polynomial time (see, e.g., [31, Theorem 48.4]).

10.3. Symmetry-forced rigidity with space group symmetry. Let $C_{\pi / 2}$ be the $2 \times 2$-matrix representing the 4 -fold rotation about the origin in $\mathbb{R}^{2}$. In 66.4 . we have seen that the idea of characterizing robust drawings with point group symmetry can be directly applied to characterizing the symmetry-forced infinitesimal rigidity of symmetric 2-dimensional frameworks if the underlying point group commutes with $C_{\pi / 2}$. Here, we show an analogous fact in space groups.

The space group $\Gamma$ we can cope with here is the case when the linear part $\Gamma_{1}$ is a group of rotations about the origin. More specifically, $\Gamma$ falls into five crystallographic group types, called p1,p2,p3, p4, p6 in terms of crystallographic notation. In the subsequent discussion, $\Gamma$ is assumed to be one of $\mathrm{p} 1, \mathrm{p} 2, \mathrm{p} 3$, p4, or p6.

Let $(H, p)$ be a $\Gamma$-symmetric framework with a $\Gamma$-symmetric graph $H$ (with a specific free action $\theta$ ) and a $\Gamma$-symmetric point-configuration $p$. Recall that an infinitesimal motion of $(H, p)$ is defined as $m: V(H) \rightarrow \mathbb{R}^{2}$ satisfying

$$
\langle m(i)-m(j), p(i)-p(j)\rangle=0 \quad\{i, j\} \in E(H) .
$$


As in the previous subsection, we are interested in $\Gamma$-symmetric motions, where we say that an infinitesimal motion $m$ is $\Gamma$-symmetric if there is $M \in \overline{\operatorname{Lat}}(\Gamma)$ such that

$$
m(\gamma v)=A_{\gamma} m(v)+M z_{\gamma} \quad \forall v \in V(H), \forall \gamma \in \Gamma
$$

(where $c_{\gamma}=0$ for any $\gamma \in \Gamma$ since $\Gamma \in\{\mathrm{p} 1, \mathrm{p} 2, \mathrm{p} 3, \mathrm{p} 4, \mathrm{p} 6\}$ ).

It can be observed that the infinitesimal rotation $m_{r}: V(H) \rightarrow \mathbb{R}^{2}$ defined by $m_{r}(v)=C_{\pi / 2} p(v)$ is always a $\Gamma$-symmetric infinitesimal motion of $(H, p)$. To see this, let $M=C_{\pi / 2} B_{\Gamma}$. Then, since $C_{\pi / 2}$ commutes with $A_{\gamma}=B_{\Gamma} K_{\gamma} B_{\Gamma}^{-1}$ for any $\gamma \in \Gamma$, we have $C_{\pi / 2} B_{\Gamma} K_{\gamma} B_{\Gamma}^{-1} C_{\pi / 2}^{-1}=B_{\Gamma} K_{\gamma} B_{\Gamma}^{-1}$, implying that $M=C_{\pi / 2} B_{\Gamma} \in$ $\overline{\operatorname{Lat}}(\Gamma)$. Moreover, for any $\gamma \in \Gamma$ and $v \in V(H)$, we have

$$
m_{r}(\gamma v)=C_{\pi / 2} p(\gamma v)=C_{\pi / 2}\left(A_{\gamma} p(v)+B_{\Gamma} z_{\gamma}\right)=A_{\gamma} m_{r}(v)+M z_{\gamma},
$$

which implies that $m_{r}$ satisfies (10.12) and is indeed a $\Gamma$-symmetric motion.

Also it is easy to see that for any $t \in \bigcap_{\gamma \in \Gamma}\left(A_{\gamma}-I_{d}\right)$, translation $m_{t}$ defined by $m_{t}(v)=t$ for $v \in V(H)$ is a $\Gamma$-symmetric motion with $M=0$.

We say that $(H, p)$ is infinitesimally rigid if every possible $\Gamma$-symmetric infinitesimal motion is a linear combination of such translations $m_{t}$ and $m_{r}$.

As usual, taking a representative vertex $v$ from each vertex orbit $\Gamma v$, (10.11) is reduced to the system

$$
\left\langle m(i)-m\left(\psi_{e} j\right), p(i)-p\left(\psi_{e} j\right)\right\rangle=0
$$

over all edge orbits from $\Gamma i$ to $\Gamma j$ with the gain $\psi_{e}$. Thus, the problem can be considered in a general $\Gamma$-gain graph $(G=(V, E), \psi)$ with $p: V \rightarrow \mathbb{R}^{2}$, and we are asked to compute the dimension of the linear space of $(m, M) \in\left(\mathbb{R}^{2}\right)^{V} \oplus \overline{\operatorname{Lat}}(\Gamma)$ satisfying

$$
\left\langle m(i)-\left(A_{\psi_{e}} m(j)+M z_{\psi_{e}}\right), p(i)-\left(A_{\psi_{e}} p(j)+t_{\psi_{e}}\right)\right\rangle=0 \quad \forall(i, j) \in E .
$$

Let $B_{1}, \ldots, B_{k} \in \mathbb{R}^{d \times d}$ be a basis of $\overline{\operatorname{Lat}}(\Gamma)$. As defined in (10.6), we shall consider a bilinear function $b_{i}: \mathbb{R}^{d} \times \mathbb{R}^{d} \rightarrow \mathbb{R}$ given by $b_{i}(\alpha, t)=\left\langle\alpha, B_{i} B_{\Gamma}^{-1} t\right\rangle$ for $(\alpha, t) \in \mathbb{R}^{d} \times \mathbb{R}^{d}$, and $b: \mathbb{R}^{d} \times \mathbb{R}^{d} \rightarrow \mathbb{R}^{k}$ given by $b=\left(b_{1}, \ldots, b_{k}\right)^{\top}$.

To analyze the system (10.14), we shall associate a 1-dimensional linear space with each $e=(i, j) \in E$ as follows:

$$
R_{e, \psi}^{\prime}(p)=U_{e, \psi} \cap\left\{x \in\left(\mathbb{R}^{2}\right)^{V} \oplus \mathbb{R}^{k} \mid\left\langle C_{\pi / 2}\left(p(i)-\left(A_{\psi_{e}} p(j)+t_{\psi_{e}}\right)\right), x(i)\right\rangle=0\right\},
$$

where $U_{e, \psi}$ is as defined in (10.8), (10.9). Then, applying the same proof as that of Lemma 10.3, it is easy to check the following.

Lemma 10.7. Let $\Gamma$ be a 2-dimensional space group whose linear part $\Gamma_{1}$ is a group of rotations, $(G=(V, E), \psi)$ a $\Gamma$-gain graph, and $p: V \rightarrow \mathbb{R}^{2}$. Then, the space of $(m, M) \in\left(\mathbb{R}^{d}\right)^{V} \oplus \overline{\operatorname{Lat}}(\Gamma)$ satisfying (10.14) is equal to

$$
2|V|+k-\operatorname{dim}_{\mathbb{R}}\left\{R_{e, \psi}^{\prime}(p) \mid e \in E\right\}
$$

where $k=\operatorname{dim}_{\mathbb{R}} \overline{\operatorname{Lat}}(\Gamma)$.

Theorem 10.8. Let $\Gamma$ be a 2-dimensional space group whose point group $\Gamma_{1}$ is a group of rotations and whose lattice is generic, and let $(G=(V, E), \psi)$ be a $\Gamma$-gain graph. Then, for almost all $p: V \rightarrow \mathbb{R}^{2}, \operatorname{dim}_{\mathbb{R}}\left\{R_{e, \psi}^{\prime}(p) \mid e \in E\right\}=\hat{h}_{\Gamma}(E)$, where $h_{\Gamma}: 2^{E} \rightarrow \mathbb{Z}$ is

$$
h_{\Gamma}(F)=2|V(F)|-2 c(F)+\operatorname{dim}_{\mathbb{R}}\left\{U_{e, \psi_{F}^{\circ}} \mid e \in E\left(G_{F}^{\circ}\right)\right\}-1 \quad(F \subseteq E) .
$$


Proof. Recall that $C_{\pi / 2} B_{\Gamma} \in \overline{\operatorname{Lat}}(\Gamma)$. Hence, there is $s=\left(s_{1}, \ldots, s_{k}\right)^{\top} \in \mathbb{R}^{k}$ such that $\sum_{i} s_{i} B_{i}=C_{\pi / 2} B_{\Gamma}$. Since the lattice is generic, $\left\{s_{1}, \ldots, s_{k}\right\}$ is algebraically independent over $\mathbb{Q}_{\Gamma_{1}}$.

Let us take any $p: V \rightarrow \mathbb{R}^{2}$ such that the coordinates of $p$ and $s_{1}, \ldots, s_{k}$ form an algebraically independent set over $\mathbb{Q}_{\Gamma_{1}}$. We define a hyperplane $\mathcal{H}^{\prime}$ of $\left(\mathbb{R}^{2}\right)^{V} \oplus \mathbb{R}^{k}$ by

$$
\mathcal{H}^{\prime}=\left\{x \in\left(\mathbb{R}^{2}\right)^{V} \oplus \mathbb{R}^{k} \mid \sum_{v \in V}\left\langle C_{\pi / 2} p(v), x(v)\right\rangle+\langle s, x(*)\rangle=0\right\} .
$$

Then, it can be shown that $R_{e, \psi}^{\prime}(p)=U_{e, \psi} \cap \mathcal{H}^{\prime}$ in the same analysis as the proof of Theorem 10.5. Also, by Theorem 9.2, $\operatorname{dim}_{\mathbb{R}}\left\{U_{e, \psi} \mid e \in F\right\}=\left(h_{\Gamma}+1\right)(F)$ for any $F \subseteq E$. Since $\mathcal{H}^{\prime}$ is generic, by Theorem $\left[3.2\right.$, we obtain $\operatorname{dim}_{\mathbb{R}}\left\{R_{e, \psi}^{\prime}(p) \mid e \in F\right\}=$ $\hat{h}_{\Gamma}(F)$ for any $F \subseteq E$.

Lemma 10.7 and Theorem 10.8 imply the following.

Corollary 10.9. Let $\Gamma$ be a 2-dimensional space group whose linear part $\Gamma_{1}$ is a group of rotations and whose lattice is generic. Let $H$ be a $\Gamma$-symmetric graph. Then, for almost all $\Gamma$-symmetric $p: V(H) \rightarrow \mathbb{R}^{2},(H, p)$ is symmetry-forced infinitesimally rigid if and only if the quotient $\Gamma$-gain graph $(G, \psi)$ contains an edge subset I satisfying the following counting conditions:

- $|I|=2|V|+k-1-\operatorname{dim}_{\mathbb{R}} \bigcap_{\gamma \in \Gamma} \operatorname{ker}\left(A_{\gamma}-I_{d}\right)$;

- $|F| \leq 2|V(F)|-2 c(F)+\operatorname{dim}_{\mathbb{R}}\left\{U_{e, \psi_{F}^{\circ}} \mid e \in E\left(G_{F}^{\circ}\right)\right\}-1$ for any nonempty $F \subseteq I$,

where $\left(G_{F}^{\circ}, \psi_{F}^{\circ}\right)$ is the compressed graph of $G$ by $F$ and $k=\operatorname{dim}_{\mathbb{R}} \overline{\operatorname{Lat}}(\Gamma)$.

For $\Gamma=\mathrm{p} 1, \mathrm{p} 2, \mathrm{p} 3, \mathrm{p} 4, \mathrm{p} 6, k=4,4,2,2,2$, respectively.

\section{ACKNOWLEDGMENTS}

The authors thank Tibor Jordán and Viktória Kaszanitzky for valuable discussions on count matroids of gain graphs. The modeling of symmetric body-bar frameworks given in $\$ 7$ is based on [5]. The authors thank Ciprian Borcea and Ileana Streinu for valuable discussions on this topic.

\section{REFERENCES}

[1] L. Asimow and B. Roth, The rigidity of graphs, Trans. Amer. Math. Soc. 245 (1978), 279-289, DOI 10.2307/1998867. MR511410 (80i:57004a)

[2] Norman Biggs, Algebraic graph theory, 2nd ed., Cambridge Mathematical Library, Cambridge University Press, Cambridge, 1993. MR,1271140 (95h:05105)

[3] Ciprian S. Borcea and Ileana Streinu, Periodic frameworks and flexibility, Proc. R. Soc. Lond. Ser. A Math. Phys. Eng. Sci. 466 (2010), no. 2121, 2633-2649, DOI 10.1098/rspa.2009.0676. MR2671687(2011k:05168)

[4] Ciprian S. Borcea and Ileana Streinu, Minimally rigid periodic graphs, Bull. Lond. Math. Soc. 43 (2011), no. 6, 1093-1103, DOI 10.1112/blms/bdr044. MR2861531 (2012m:52036)

[5] Ciprian Borcea, Ileana Streinu, and Shin-ichi Tanigawa, Periodic body-and-bar frameworks, SIAM J. Discrete Math. 29 (2015), no. 1, 93-112, DOI 10.1137/120900265. MR3300404

[6] R. Connelly, P. W. Fowler, S. D. Guest, B. Schulze, and W. J. Whiteley, When is a symmetric pin-jointed framework isostatic?, Int. J. Solids Struct. 46 (2009), no. 3-4, 762-773.

[7] T. A. Dowling, A class of geometric lattices based on finite groups, J. Combinatorial Theory Ser. B 14 (1973), 61-86. MR.0307951 (46 \#7066)

[8] Jack Edmonds, Matroid partition, Mathematics of the Decision Sciences, Part I (Seminar, Stanford, Calif., 1967), Amer. Math. Soc., Providence, R.I., 1968, pp. 335-345. MR0237366 (38 \#5654) 
[9] Jack Edmonds, Submodular functions, matroids, and certain polyhedra, Combinatorial Structures and their Applications (Proc. Calgary Internat. Conf., Calgary, Alta., 1969), Gordon and Breach, New York, 1970, pp. 69-87. MR0270945 (42 \#5828)

[10] P. W. Fowler and S. D. Guest, A symmetry extension of Maxwell's rule for rigidity of frames, Internat. J. Solids Structures 37 (1999), no. 12, 1793-1804, DOI 10.1016/S00207683(98)00326-6. MR.1804455 (2001j:74053)

[11] András Frank, Connections in combinatorial optimization, Oxford Lecture Series in Mathematics and its Applications, vol. 38, Oxford University Press, Oxford, 2011. MR2848535 (2012i:90003)

[12] Satoru Fujishige, Submodular functions and optimization, 2nd ed., Annals of Discrete Mathematics, vol. 58, Elsevier B. V., Amsterdam, 2005. MR2171629 (2006d:90098)

[13] Herman Gluck, Almost all simply connected closed surfaces are rigid, Geometric topology (Proc. Conf., Park City, Utah, 1974), Springer, Berlin, 1975, pp. 225-239. Lecture Notes in Math., Vol. 438. MR0400239 (53 \#4074)

[14] Jonathan L. Gross and Thomas W. Tucker, Topological graph theory, Wiley-Interscience Series in Discrete Mathematics and Optimization, John Wiley \& Sons, Inc., New York, 1987. MR898434 (88h:05034)

[15] W. V. D. Hodge and D. Pedoe, Methods of algebraic geometry. Vol. I, Cambridge Mathematical Library, Cambridge University Press, Cambridge, 1994. Book I: Algebraic preliminaries; Book II: Projective space; Reprint of the 1947 original. MR1288305 (95d:14002a)

[16] Bill Jackson and Tibor Jordán, Globally rigid circuits of the direction-length rigidity matroid, J. Combin. Theory Ser. B 100 (2010), no. 1, 1-22, DOI 10.1016/j.jctb.2009.03.004. MR2563511(2011c:05078)

[17] T. Jordán, V. E. Kaszanitzky, and S. Tanigawa, Gain-sparsity and symmetric rigidity in the plane, EGRES Technical Report TR 2012-17.

[18] G. Laman, On graphs and rigidity of plane skeletal structures, J. Engrg. Math. 4 (1970), 331-340. MR0269535 (42 \#4430)

[19] L. Lovász, Flats in matroids and geometric graphs, Combinatorial surveys (Proc. Sixth British Combinatorial Conf., Royal Holloway Coll., Egham, 1977), Academic Press, London, 1977, pp. 45-86. MR.0480111 (58 \#310)

[20] L. Lovász and Y. Yemini, On generic rigidity in the plane, SIAM J. Algebraic Discrete Methods 3 (1982), no. 1, 91-98, DOI 10.1137/0603009. MR644960 (83b:52007)

[21] J. Malestein and L. Theran, Generic rigidity of frameworks with orientation-preserving crystallographic symmetry, arXiv:1108.2518 (2011).

[22] Justin Malestein and Louis Theran, Generic combinatorial rigidity of periodic frameworks, Adv. Math. 233 (2013), 291-331, DOI 10.1016/j.aim.2012.10.007. MR2995673

[23] J. H. Mason, Matroids as the study of geometrical configurations, Higher combinatorics (Proc. NATO Advanced Study Inst., Berlin, 1976), NATO Adv. Study Inst. Ser., Ser. C: Math. Phys. Sci., vol. 31, Reidel, Dordrecht-Boston, Mass., 1977, pp. 133-176. MR.519783 (80k:05037)

[24] J. H. Mason, Glueing matroids together: a study of Dilworth truncations and matroid analogues of exterior and symmetric powers, Algebraic methods in graph theory, Vol. I, II (Szeged, 1978), Colloq. Math. Soc. János Bolyai, vol. 25, North-Holland, Amsterdam-New York, 1981, pp. 519-561. MR642060 (84i:05041)

[25] J. C. Owen and S. C. Power, Infinite bar-joint frameworks, crystals and operator theory, New York J. Math. 17 (2011), 445-490. MR2831050 (2012g:52041)

[26] J. C. Owen and S. C. Power, Frameworks symmetry and rigidity, Internat. J. Comput. Geom. Appl. 20 (2010), no. 6, 723-750, DOI 10.1142/S0218195910003505. MR2747433 (2011k:52032)

[27] James Oxley, Matroid theory, 2nd ed., Oxford Graduate Texts in Mathematics, vol. 21, Oxford University Press, Oxford, 2011. MR2849819 (2012k:05002)

[28] S. C. Power, Polynomials for crystal frameworks and the rigid unit mode spectrum, Phil. Trans. R. Soc. A. 372 (2014), 20120030.

[29] Elissa Ross, Geometric and combinatorial rigidity of periodic frameworks as graphs on the torus, ProQuest LLC, Ann Arbor, MI. Thesis (Ph.D.)-York University (Canada), 2011. MR2941979

[30] Elissa Ross, The rigidity of periodic body-bar frameworks on the three-dimensional fixed torus, Philos. Trans. R. Soc. Lond. Ser. A Math. Phys. Eng. Sci. 372 (2014), no. 2008, 20120112, 23, DOI 10.1098/rsta.2012.0112. MR3158335 
[31] A. Schrijver, Combinatorial optimization: polyhedra and efficiency, Vols. A-C, Springer, 2003. MR 1956924, MR.1956925, MR.1956926

[32] B. Schulze, Combinatorial and geometric rigidity with symmetric constraints, Ph. thesis, York University, 2009.

[33] Bernd Schulze, Symmetric versions of Laman's theorem, Discrete Comput. Geom. 44 (2010), no. 4, 946-972, DOI 10.1007/s00454-009-9231-x. MR2728043(2011j:52053)

[34] Bernd Schulze, Symmetry as a sufficient condition for a finite flex, SIAM J. Discrete Math. 24 (2010), no. 4, 1291-1312, DOI 10.1137/090776238. MR2735924 (2011j:52054)

[35] Bernd Schulze, Adnan Sljoka, and Walter Whiteley, How does symmetry impact the flexibility of proteins?, Philos. Trans. R. Soc. Lond. Ser. A Math. Phys. Eng. Sci. 372 (2014), no. 2008, 20120041, 20, DOI 10.1098/rsta.2012.0041. MR.3158333

[36] Bernd Schulze and Walter Whiteley, The orbit rigidity matrix of a symmetric framework, Discrete Comput. Geom. 46 (2011), no. 3, 561-598, DOI 10.1007/s00454-010-9317-5. MR2826970 (2012k:52055)

[37] Brigitte Servatius and Walter Whiteley, Constraining plane configurations in computer-aided design: combinatorics of directions and lengths, SIAM J. Discrete Math. 12 (1999), no. 1, 136-153 (electronic), DOI 10.1137/S0895480196307342. MR1666077 (99m:68218)

[38] Shin-ichi Tanigawa, Generic rigidity matroids with Dilworth truncations, SIAM J. Discrete Math. 26 (2012), no. 3, 1412-1439, DOI 10.1137/100819473. MR3022145

[39] Tiong-Seng Tay, Rigidity of multigraphs. I. Linking rigid bodies in $n$-space, J. Combin. Theory Ser. B 36 (1984), no. 1, 95-112, DOI 10.1016/0095-8956(84)90016-9. MR742389 (85i:05205)

[40] Walter Whiteley, The union of matroids and the rigidity of frameworks, SIAM J. Discrete Math. 1 (1988), no. 2, 237-255, DOI 10.1137/0401025. MR941354 (89d:05055)

[41] Walter Whiteley, A matroid on hypergraphs, with applications in scene analysis and geometry, Discrete Comput. Geom. 4 (1989), no. 1, 75-95, DOI 10.1007/BF02187716. MR.964145 (89k:05027)

[42] Walter Whiteley, Some matroids from discrete applied geometry, Matroid theory (Seattle, WA, 1995), Contemp. Math., vol. 197, Amer. Math. Soc., Providence, RI, 1996, pp. 171-311, DOI 10.1090/conm/197/02540. MR/1411692(97h:05040)

[43] Geoff Whittle, A generalisation of the matroid lift construction, Trans. Amer. Math. Soc. 316 (1989), no. 1, 141-159, DOI 10.2307/2001277. MR957084(90b:05038)

[44] Thomas Zaslavsky, Biased graphs. I. Bias, balance, and gains, J. Combin. Theory Ser. B 47 (1989), no. 1, 32-52, DOI 10.1016/0095-8956(89)90063-4. MR1007712 (90k:05138)

[45] Thomas Zaslavsky, Biased graphs. II. The three matroids, J. Combin. Theory Ser. B 51 (1991), no. 1, 46-72, DOI 10.1016/0095-8956(91)90005-5. MR 1088626 (91m:05056)

[46] Thomas Zaslavsky, Frame matroids and biased graphs, European J. Combin. 15 (1994), no. 3, 303-307, DOI 10.1006/eujc.1994.1034. MR.1273951 (95a:05021)

[47] Thomas Zaslavsky, Biased graphs. IV. Geometrical realizations, J. Combin. Theory Ser. B 89 (2003), no. 2, 231-297, DOI 10.1016/S0095-8956(03)00035-2. MR2017726 (2005b:05057)

Research Institute for Mathematical Sciences, Kyoto University, Kyoto 606-8502, JAPAN

E-mail address: tanigawa@kurims.kyoto-u.ac.jp 